Supporting Information

\title{
Design, Synthesis, and Insecticidal Activity of Novel Doramectin
}

\section{Derivatives Containing Acylurea and Acylthiourea Based on}

\section{Hydrogen Bonding}

Qi Zhang ${ }^{\mathrm{a}, \mathrm{b},+}$, Yao Cheng ${ }^{\mathrm{a}, \mathrm{c},+}$, Cheng Zheng ${ }^{\mathrm{a}, \mathrm{c}}$, Ping Bai ${ }^{\mathrm{a}, \mathrm{c}}$, Jian Yang ${ }^{\mathrm{a}, \mathrm{c}}$, and Xiaoxia $\mathrm{Lu}^{\mathrm{a}, \mathrm{c}^{*}}$

a Chengdu Institute of Biology, Chinese Academy of Sciences, Chengdu 610041, People's Republic of China.

${ }^{\mathrm{b}}$ Department of Chemical and Chemical Engineering, Hefei Normal University, Hefei, 230601, People's Republic of China.

${ }^{c}$ University of Chinese Academy of Sciences, Beijing 100049, People's Republic of China.

${ }^{+}$These authors contributed equally to this paper.

Supporting Information Available. The data and characterization of all compounds were given.

\section{Supplementary Data}

S-1: Structure and HRMS of Target Compounds-----------------------------------------5

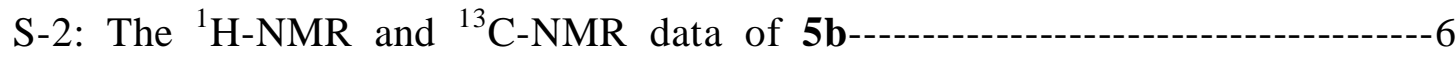

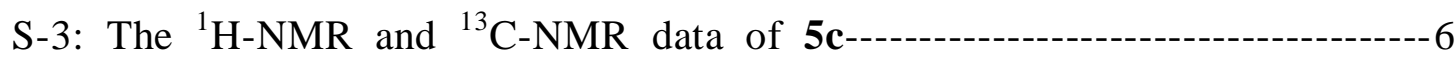

S-4: The ${ }^{1} \mathrm{H}-\mathrm{NMR}$ and ${ }^{13} \mathrm{C}-\mathrm{NMR}$ data of $\mathbf{5 d -}$

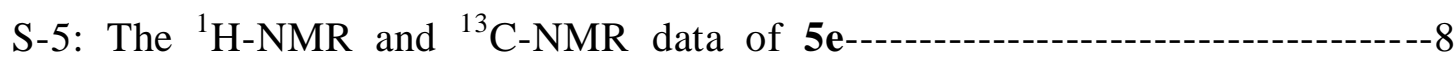

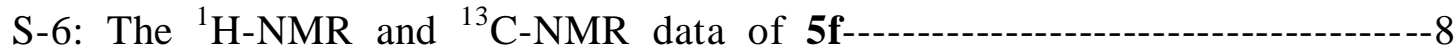

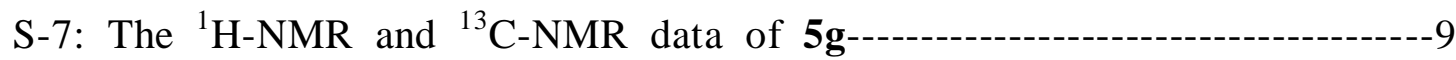

S-8: The ${ }^{1} \mathrm{H}-\mathrm{NMR}$ and ${ }^{13} \mathrm{C}-\mathrm{NMR}$ data of $\mathbf{5 h}---$

S-9: The ${ }^{1} \mathrm{H}-\mathrm{NMR}$ and ${ }^{13} \mathrm{C}-\mathrm{NMR}$ data of $\mathbf{5 i}$----------------------------------10

S-10: The ${ }^{1} \mathrm{H}-\mathrm{NMR}$ and ${ }^{13} \mathrm{C}-\mathrm{NMR}$ data of $\mathbf{5 j - - - - - - - - - - - - - - - - - - - - - - - - - - - - - - - - - - 1 1}$

S-11: The ${ }^{1} \mathrm{H}-\mathrm{NMR}$ and ${ }^{13} \mathrm{C}-\mathrm{NMR}$ data of $\mathbf{5 k}$-----------------------------------12 
S-12: The ${ }^{1} \mathrm{H}-\mathrm{NMR}$ and ${ }^{13} \mathrm{C}-\mathrm{NMR}$ data of $5 \mathrm{l}-$

S-13: The ${ }^{1} \mathrm{H}-\mathrm{NMR}$ and ${ }^{13} \mathrm{C}-\mathrm{NMR}$ data of $\mathbf{5 m -}$

S-14: The ${ }^{1} \mathrm{H}-\mathrm{NMR}$ and ${ }^{13} \mathrm{C}-\mathrm{NMR}$ data of $\mathbf{5 n}$--

S-15: The ${ }^{1} \mathrm{H}-\mathrm{NMR}$ and ${ }^{13} \mathrm{C}-\mathrm{NMR}$ data of $\mathbf{5 0}----------------------------14$

S-16: The ${ }^{1} \mathrm{H}-\mathrm{NMR}$ and ${ }^{13} \mathrm{C}-\mathrm{NMR}$ data of $\mathbf{5 p}$---------------------------15

S-17: The ${ }^{1} \mathrm{H}-\mathrm{NMR}$ and ${ }^{13} \mathrm{C}-\mathrm{NMR}$ data of $\mathbf{5 q}$

S-18: The ${ }^{1} \mathrm{H}-\mathrm{NMR}$ and ${ }^{13} \mathrm{C}-\mathrm{NMR}$ data of $5 \mathbf{r}$--

S-19: The ${ }^{1} \mathrm{H}-\mathrm{NMR}$ and ${ }^{13} \mathrm{C}-\mathrm{NMR}$ data of $\mathbf{5 s -}$

S-20: The ${ }^{1} \mathrm{H}-\mathrm{NMR}$ and ${ }^{13} \mathrm{C}-\mathrm{NMR}$ data of $\mathbf{5 t - 1 -}$

S-21: The ${ }^{1} \mathrm{H}-\mathrm{NMR}$ and ${ }^{13} \mathrm{C}-\mathrm{NMR}$ data of $\mathbf{5 u} \mathbf{-}$

S-22: The ${ }^{1} \mathrm{H}-\mathrm{NMR}$ and ${ }^{13} \mathrm{C}-\mathrm{NMR}$ data of $\mathbf{5 v}$--

S-23: The ${ }^{1} \mathrm{H}-\mathrm{NMR}$ and ${ }^{13} \mathrm{C}-\mathrm{NMR}$ data of $\mathbf{6 b}-$

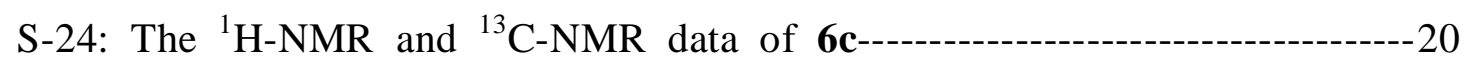

S-25: The ${ }^{1} \mathrm{H}-\mathrm{NMR}$ and ${ }^{13} \mathrm{C}-\mathrm{NMR}$ data of $\mathbf{6 d -}$

S-26: The ${ }^{1} \mathrm{H}-\mathrm{NMR}$ and ${ }^{13} \mathrm{C}-\mathrm{NMR}$ data of $6 \mathrm{e}---$

S-27: The ${ }^{1} \mathrm{H}-\mathrm{NMR}$ and ${ }^{13} \mathrm{C}-\mathrm{NMR}$ data of $6 \mathbf{f}-$

S-28: The ${ }^{1} \mathrm{H}-\mathrm{NMR}$ and ${ }^{13} \mathrm{C}-\mathrm{NMR}$ data of $\mathbf{6 g}$--

S-29: The ${ }^{1} \mathrm{H}-\mathrm{NMR}$ and ${ }^{13} \mathrm{C}-\mathrm{NMR}$ data of $\mathbf{6 h}--------$

S-30: The ${ }^{1} \mathrm{H}-\mathrm{NMR}$ and ${ }^{13} \mathrm{C}-\mathrm{NMR}$ data of $\mathbf{6 i -}$

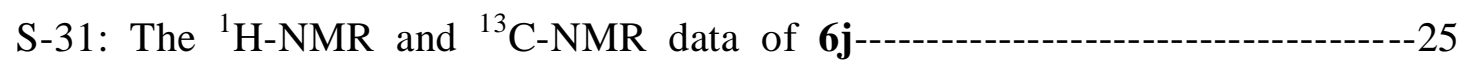

S-32: The ${ }^{1} \mathrm{H}-\mathrm{NMR}$ and ${ }^{13} \mathrm{C}-\mathrm{NMR}$ data of $6 \mathbf{k}-$

S-33: The ${ }^{1} \mathrm{H}-\mathrm{NMR}$ and ${ }^{13} \mathrm{C}-\mathrm{NMR}$ data of $61--\ldots$

S-34: The ${ }^{1} \mathrm{H}-\mathrm{NMR}$ and ${ }^{13} \mathrm{C}-\mathrm{NMR}$ data of $\mathbf{6 m}-$

S-35: The ${ }^{1} \mathrm{H}-\mathrm{NMR}$ and ${ }^{13} \mathrm{C}-\mathrm{NMR}$ data of $\mathbf{6 n}$--_-

S-36: The ${ }^{1} \mathrm{H}-\mathrm{NMR}$ and ${ }^{13} \mathrm{C}-\mathrm{NMR}$ data of $\mathbf{6 0}-$

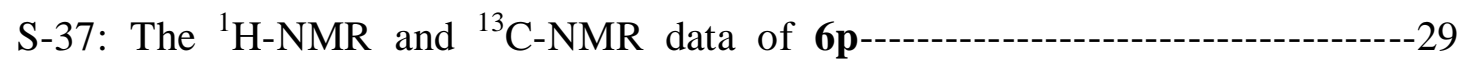

S-38: The ${ }^{1} \mathrm{H}-\mathrm{NMR}$ and ${ }^{13} \mathrm{C}-\mathrm{NMR}$ data of $\mathbf{6 q}$----------29

S-39: The ${ }^{1} \mathrm{H}-\mathrm{NMR}$ and ${ }^{13} \mathrm{C}-\mathrm{NMR}$ data of $6 r-$

S-40: The ${ }^{1} \mathrm{H}-\mathrm{NMR}$ and ${ }^{13} \mathrm{C}-\mathrm{NMR}$ data of $6 \mathrm{~s}-$

S-41: The ${ }^{1} \mathrm{H}-\mathrm{NMR}$ and ${ }^{13} \mathrm{C}-\mathrm{NMR}$ data of $6 \mathrm{t}$ 
S-42: The ${ }^{1} \mathrm{H}-\mathrm{NMR}$ and ${ }^{13} \mathrm{C}-\mathrm{NMR}$ data of $6 \mathrm{u}-------------------------------------32$

S-43: The ${ }^{1} \mathrm{H}-\mathrm{NMR}$ and ${ }^{13} \mathrm{C}-\mathrm{NMR}$ data of $6 \mathrm{v}-----------------------------------33$

S-44: The ${ }^{1} \mathrm{H}-\mathrm{NMR}$ and ${ }^{13} \mathrm{C}-\mathrm{NMR}$ spectrums of $\mathbf{5 a} \mathbf{a}-----------------------------------34$

S-45: The ${ }^{1} \mathrm{H}-\mathrm{NMR}$ and ${ }^{13} \mathrm{C}-\mathrm{NMR}$ spectrums of $\mathbf{5 b} \mathbf{b}-------------------------------------35$

S-46: The ${ }^{1} \mathrm{H}-\mathrm{NMR}$ and ${ }^{13} \mathrm{C}-\mathrm{NMR}$ spectrums of $\mathbf{5 c}-----------------------------------36$

S-47: The ${ }^{1} \mathrm{H}-\mathrm{NMR}$ and ${ }^{13} \mathrm{C}-\mathrm{NMR}$ spectrums of $\mathbf{5 d}$-----------------------------------37

S-48: The ${ }^{1} \mathrm{H}-\mathrm{NMR}$ and ${ }^{13} \mathrm{C}-\mathrm{NMR}$ spectrums of $\mathbf{5 e}----------------------------------38$

S-49: The ${ }^{1} \mathrm{H}-\mathrm{NMR}$ and ${ }^{13} \mathrm{C}-\mathrm{NMR}$ spectrums of $\mathbf{5 f}$------------------------------------39

S-50: The ${ }^{1} \mathrm{H}-\mathrm{NMR}$ and ${ }^{13} \mathrm{C}-\mathrm{NMR}$ spectrums of $\mathbf{5 g}$-------------------------------40

S-51: The ${ }^{1} \mathrm{H}-\mathrm{NMR}$ and ${ }^{13} \mathrm{C}-\mathrm{NMR}$ spectrums of $\mathbf{5 h}$----------------------------------41

S-52: The ${ }^{1} \mathrm{H}-\mathrm{NMR}$ and ${ }^{13} \mathrm{C}-\mathrm{NMR}$ spectrums of $\mathbf{5 i}$----------------------------------42

S-53: The ${ }^{1} \mathrm{H}-\mathrm{NMR}$ and ${ }^{13} \mathrm{C}-\mathrm{NMR}$ spectrums of $\mathbf{5 j - - - - - - - - - - - - - - - - - - - - - - - - - - - - - - - - - - - - 4 3}$

S-54: The ${ }^{1} \mathrm{H}-\mathrm{NMR}$ and ${ }^{13} \mathrm{C}-\mathrm{NMR}$ spectrums of $\mathbf{5 k - - - - - - - - - - - - - - - - - - - - - - - - - - - - - - - - - - - - 4 4}$

S-55: The ${ }^{1} \mathrm{H}-\mathrm{NMR}$ and ${ }^{13} \mathrm{C}-\mathrm{NMR}$ spectrums of 5l----------------------------------45

S-56: The ${ }^{1} \mathrm{H}-\mathrm{NMR}$ and ${ }^{13} \mathrm{C}-\mathrm{NMR}$ spectrums of $\mathbf{5 m - - - - - - - - - - - - - - - - - - - - - - - - - - - - - - - - - - - - 4 6}$

S-57: The ${ }^{1} \mathrm{H}-\mathrm{NMR}$ and ${ }^{13} \mathrm{C}-\mathrm{NMR}$ spectrums of $\mathbf{5 n - - - - - - - - - - - - - - - - - - - - - - - - - - - - - - - - - - - - 4 7}$

S-58: The ${ }^{1} \mathrm{H}-\mathrm{NMR}$ and ${ }^{13} \mathrm{C}-\mathrm{NMR}$ spectrums of $\mathbf{5 0}$

S-59: The ${ }^{1} \mathrm{H}-\mathrm{NMR}$ and ${ }^{13} \mathrm{C}-\mathrm{NMR}$ spectrums of $\mathbf{5 p}$--------------------------------49

S-60: The ${ }^{1} \mathrm{H}-\mathrm{NMR}$ and ${ }^{13} \mathrm{C}-\mathrm{NMR}$ spectrums of $\mathbf{5 q}$------------------------------------50

S-61: The ${ }^{1} \mathrm{H}-\mathrm{NMR}$ and ${ }^{13} \mathrm{C}-\mathrm{NMR}$ spectrums of $\mathbf{5 r}$---------------------------------51

S-62: The ${ }^{1} \mathrm{H}-\mathrm{NMR}$ and ${ }^{13} \mathrm{C}-\mathrm{NMR}$ spectrums of $\mathbf{5 s - - - - - - - - - - - - - - - - - - - - - - - - - - - - - - - - - - - 5 2}$

S-63: The ${ }^{1} \mathrm{H}-\mathrm{NMR}$ and ${ }^{13} \mathrm{C}-\mathrm{NMR}$ spectrums of $\mathbf{5 t}$------------------------------------53

S-64: The ${ }^{1} \mathrm{H}-\mathrm{NMR}$ and ${ }^{13} \mathrm{C}-\mathrm{NMR}$ spectrums of $\mathbf{5 u}$----------------------------------54

S-65: The ${ }^{1} \mathrm{H}-\mathrm{NMR}$ and ${ }^{13} \mathrm{C}-\mathrm{NMR}$ spectrums of $\mathbf{5 v - - - - - - - - - - - - - - - - - - - - - - - - - - - - - - - - - - - 5 5}$

S-66: The ${ }^{1} \mathrm{H}-\mathrm{NMR}$ and ${ }^{13} \mathrm{C}-\mathrm{NMR}$ spectrums of $\mathbf{6 a} \mathbf{a}-------------------------------------56$

S-67: The ${ }^{1} \mathrm{H}-\mathrm{NMR}$ and ${ }^{13} \mathrm{C}-\mathrm{NMR}$ spectrums of $\mathbf{6 b}----------------------------------57$

S-68: The ${ }^{1} \mathrm{H}-\mathrm{NMR}$ and ${ }^{13} \mathrm{C}-\mathrm{NMR}$ spectrums of $\mathbf{6 c - - - - - - - - - - - - - - - - - - - - - - - - - - - - - - - 5 8}$

S-69: The ${ }^{1} \mathrm{H}-\mathrm{NMR}$ and ${ }^{13} \mathrm{C}-\mathrm{NMR}$ spectrums of $\mathbf{6 d - - - - - - - - - - - - - - - - - - - - - - - - - - - - - - - - - - - - 5 9}$

S-70: The ${ }^{1} \mathrm{H}-\mathrm{NMR}$ and ${ }^{13} \mathrm{C}-\mathrm{NMR}$ spectrums of $6 \mathrm{e}---------------------------------60$

S-71: The ${ }^{1}$ H-NMR and ${ }^{13}$ C-NMR spectrums of $6 f------------------------------------61$ 
S-72: The ${ }^{1} \mathrm{H}-\mathrm{NMR}$ and ${ }^{13} \mathrm{C}-\mathrm{NMR}$ spectrums of $\mathbf{6 g}-------------------------------------62$

S-73: The ${ }^{1} \mathrm{H}-\mathrm{NMR}$ and ${ }^{13} \mathrm{C}-\mathrm{NMR}$ spectrums of $\mathbf{6 h}---------------------------------63$

S-74: The ${ }^{1} \mathrm{H}-\mathrm{NMR}$ and ${ }^{13} \mathrm{C}-\mathrm{NMR}$ spectrums of $\mathbf{6 i -}----------------------------------64$

S-75: The ${ }^{1} \mathrm{H}-\mathrm{NMR}$ and ${ }^{13} \mathrm{C}-\mathrm{NMR}$ spectrums of $\mathbf{6 j}$------------------------------------65

S-76: The ${ }^{1} \mathrm{H}-\mathrm{NMR}$ and ${ }^{13} \mathrm{C}-\mathrm{NMR}$ spectrums of $\mathbf{6 k - - - - - - - - - - - - - - - - - - - - - - - - - - - - - - - - - - - 6 6}$

S-77: The ${ }^{1} \mathrm{H}-\mathrm{NMR}$ and ${ }^{13} \mathrm{C}-\mathrm{NMR}$ spectrums of 61-------------------------------------67

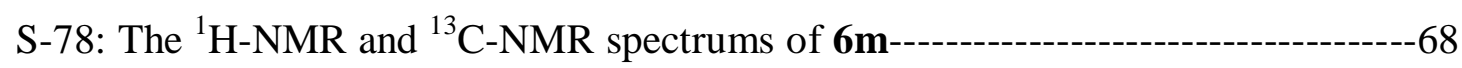

S-79: The ${ }^{1} \mathrm{H}-\mathrm{NMR}$ and ${ }^{13} \mathrm{C}-\mathrm{NMR}$ spectrums of $6 \mathbf{n}------------------------------------69$

S-80: The ${ }^{1} \mathrm{H}-\mathrm{NMR}$ and ${ }^{13} \mathrm{C}-\mathrm{NMR}$ spectrums of $6 \mathbf{0}----------------------------------70$

S-81: The ${ }^{1} \mathrm{H}-\mathrm{NMR}$ and ${ }^{13} \mathrm{C}-\mathrm{NMR}$ spectrums of $\mathbf{6 p}$----------------------------------71

S-82: The ${ }^{1} \mathrm{H}-\mathrm{NMR}$ and ${ }^{13} \mathrm{C}-\mathrm{NMR}$ spectrums of $\mathbf{6 q -}-----------------------------------72$

S-83: The ${ }^{1} \mathrm{H}-\mathrm{NMR}$ and ${ }^{13} \mathrm{C}-\mathrm{NMR}$ spectrums of $6 \mathbf{r}-------------------------------------73$

S-84: The ${ }^{1} \mathrm{H}-\mathrm{NMR}$ and ${ }^{13} \mathrm{C}-\mathrm{NMR}$ spectrums of 6s------------------------------74

S-85: The ${ }^{1} \mathrm{H}-\mathrm{NMR}$ and ${ }^{13} \mathrm{C}-\mathrm{NMR}$ spectrums of $6 \mathrm{t}------------------------------------75$

S-86: The ${ }^{1} \mathrm{H}-\mathrm{NMR}$ and ${ }^{13} \mathrm{C}-\mathrm{NMR}$ spectrums of $6 \mathbf{u}------------------------------------76$

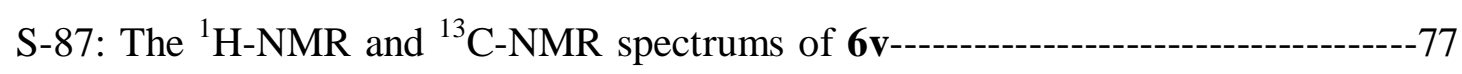




\section{S-1: Structure and HRMS of Target Compounds.}

\begin{tabular}{|c|c|c|c|c|c|c|c|}
\hline \multicolumn{2}{|c|}{ Compd. Yield } & \multirow{2}{*}{$\frac{\mathrm{m} . \mathrm{p} \cdot\left({ }^{\circ} \mathrm{C}\right)}{181-183}$} & \multicolumn{2}{|l|}{ HRMS } & \multirow{2}{*}{$\frac{\text { Yield }}{70 \%}$} & \multirow{2}{*}{$\frac{\text { m.p. }\left({ }^{\circ} \mathrm{C}\right)}{168-170}$} & HRMS \\
\hline $5 \mathbf{a}$ & $78 \%$ & & calcd.1067.5451; found 1067.5416 & $6 \mathbf{a}$ & & & calcd. 1083.5222 ; found 1083.5187 \\
\hline $5 \mathbf{b}$ & $68 \%$ & $177-178$ & calcd. 1085.5357; found 1085.5351 & $\mathbf{6 b}$ & $75 \%$ & $172-174$ & calcd. 1101.5128; found 1101.5128 \\
\hline $5 c$ & $50 \%$ & $181-183$ & calcd. $1101.5061 ;$ found 1101.5050 & $6 \mathbf{c}$ & $63 \%$ & $172-174$ & calcd. 1117.4833; found 1117.4824 \\
\hline 5d & $44 \%$ & $183-185$ & calcd. $1135.5325 ;$ found 1135.5294 & 6d & $49 \%$ & $168-169$ & calcd. 1151.5096; found 1151.5108 \\
\hline $5 e$ & $51 \%$ & 188-189 & calcd.1081.5607; found 1081.5584 & $6 e$ & $52 \%$ & 167-169 & calcd. 1097.5379; found 1097.5381 \\
\hline $\mathbf{5 f}$ & $65 \%$ & $187-188$ & calcd. 1135.5325; found 1135.5294 & 6f & $90 \%$ & $177-179$ & calcd. 1151.5096; found 1151.5121 \\
\hline $5 \mathrm{~g}$ & $69 \%$ & $184-186$ & calcd. 1085.5357; found 1085.5228 & $6 \mathrm{~g}$ & $65 \%$ & $170-172$ & calcd. 1101.5128; found 1101.5126 \\
\hline $5 \mathbf{h}$ & $91 \%$ & 195-196 & calcd. $1101.5061 ;$ found 1101.5049 & 6h & $57 \%$ & $186-188$ & calcd. 1117.4833; found 1117.4827 \\
\hline $5 \mathbf{i}$ & $55 \%$ & 198-199 & calcd. 1145.4556; found 1145.4541 & $6 \mathbf{i}$ & $69 \%$ & $170-171$ & calcd. 1161.4328; found 1161.4334 \\
\hline $5 \mathbf{j}$ & $37 \%$ & $186-187$ & calcd. 1097.5557; found 1097.5551 & $6 \mathbf{j}$ & $53 \%$ & $182-184$ & calcd. 1113.5328; found 1113.5338 \\
\hline $5 \mathbf{k}$ & $59 \%$ & 194-195 & calcd. $1135.5325 ;$ found 1135.5297 & $6 \mathbf{k}$ & $73 \%$ & $172-174$ & calcd. 1151.5096; found 1151.5074 \\
\hline 51 & $71 \%$ & $178-179$ & calcd. 1005.5294; found 1005.5311 & 61 & $70 \%$ & $154-156$ & calcd. 1021.5066; found 1021.5065 \\
\hline $5 \mathbf{m}$ & $45 \%$ & $175-176$ & calcd. 1019.5451 ; found 1019.5423 & $6 \mathrm{~m}$ & $63 \%$ & $152-154$ & calcd. $1035.5222 ;$ found 1035.5216 \\
\hline $5 n$ & $62 \%$ & $180-181$ & calcd. 1033.5607; found 1033.5622 & 6n & $39 \%$ & $166-167$ & calcd. 1049.5379; found 1049.5401 \\
\hline 50 & $61 \%$ & $183-184$ & calcd. 1031.5451 ; found 1031.5425 & 60 & $51 \%$ & $162-164$ & calcd. 1047.5222; found 1047.5228 \\
\hline $5 p$ & $89 \%$ & $180-181$ & calcd. 1073.5920; found 1073.5944 & $6 p$ & $62 \%$ & $162-163$ & calcd. 1089.5692; found 1089.5670 \\
\hline $5 q$ & $91 \%$ & $176-177$ & calcd. 1061.5920; found 1061.5892 & 6q & $49 \%$ & 153-154 & calcd. 1077.5692; found 1077.5705 \\
\hline $5 \mathbf{r}$ & $60 \%$ & 186-187 & calcd. $1103.5262 ;$ found 1103.5252 & $6 \mathbf{r}$ & $58 \%$ & $173-174$ & calcd. $1119.5034 ;$ found 1119.5055 \\
\hline $5 \mathrm{~s}$ & $82 \%$ & $185-187$ & calcd. $1103.5262 ;$ found 1103.5263 & $6 s$ & $39 \%$ & $170-172$ & calcd. 1119.5034; found 1119.5067 \\
\hline $5 t$ & $68 \%$ & $177-178$ & calcd. $1103.5262 ;$ found 1103.5264 & $6 t$ & $47 \%$ & $167-168$ & calcd. 1119.5034; found 1119.5033 \\
\hline $5 \mathbf{u}$ & $77 \%$ & $176-178$ & calcd. $1127.5662 ;$ found 1127.5678 & $6 u$ & $43 \%$ & $178-179$ & calcd. $1143.5434 ;$ found 1143.5443 \\
\hline $5 v$ & $63 \%$ & $177-179$ & calcd. 1157.5768 ; found 1157.5750 & $6 \mathbf{v}$ & $73 \%$ & $176-177$ & calcd. 1173.5539; found 1173.5521 \\
\hline
\end{tabular}


S-2: The ${ }^{1} \mathrm{H}-\mathrm{NMR}$ and ${ }^{13} \mathrm{C}-\mathrm{NMR}$ data of $\mathbf{5 b}$ :

${ }^{1} \mathrm{H}$ NMR (400 MHz, $\left.\mathrm{CDCl}_{3}\right) \delta 8.85(\mathrm{~d}, J=9.9 \mathrm{~Hz}, 1 \mathrm{H}, \mathrm{CONHCO}), 8.58(\mathrm{~d}, J=$ $14.6 \mathrm{~Hz}, 1 \mathrm{H}, \mathrm{CONH}), 8.09$ (td, $J=7.9,1.9 \mathrm{~Hz}, 1 \mathrm{H}, \mathrm{Ph}), 7.63-7.53$ (m, 1H, Ph), 7.32 (td, $J=7.7,1.1 \mathrm{~Hz}, 1 \mathrm{H}, \mathrm{Ph}), 7.23-7.15$ (m, 1H, Ph), 5.93-5.86 (m, 1H, H9), 5.83-5.68 (m, 3H, H10, H11, H23), 5.54 (dd, $J=9.9,2.5$ Hz, 1H, H22), 5.51-5.37 (m, 3H, H3, H19, H1"), 5.01 (d, $J=10.7 \mathrm{~Hz}, 1 \mathrm{H}, \mathrm{H} 15), 4.79$ (d, $J=3.8 \mathrm{~Hz}, 1 \mathrm{H}, \mathrm{H} 1$ '), 4.74-4.63 (m, 2H, H8a), 4.40 (dd, $\left.J=10.2,3.5 \mathrm{~Hz}, 1 \mathrm{H}, \mathrm{H} 4^{\prime \prime}\right), 4.30$ (d, $\left.J=6.3 \mathrm{~Hz}, 1 \mathrm{H}, \mathrm{H} 5\right)$, 4.17-4.08 (m, 1H, H5"), 4.05 (s, 1H, H7-OH), 3.98 (d, J = 6.2 Hz, 1H, H6), 3.94 (s, 1H, H13), 3.91-3.81 (m, 2H, H17, H5'), 3.74 (dt, $J=12.2,4.4$ Hz, 1H, H3"), 3.62 (dq, $J=12.7,4.7 \mathrm{~Hz}, 1 \mathrm{H}, \mathrm{H} 3$ ), 3.45 (s, 3H, H3'-OMe), 3.44 (s, 3H, H3"'-OMe), 3.34-3.23 (m, 3H, H2, H25, H4'), 2.53 (t, $J=7.8$ Hz, 1H, H12), 2.36 -2.19 (m, 5H, H5-OH, H16, H24, H2"a), 2.11-1.95 (m, 2H, H2'a, H20a), 1.88 (t, $J=2.0 \mathrm{~Hz}, 3 \mathrm{H}, \mathrm{H} 4 \mathrm{a}-\mathrm{CH}_{3}$ ), 1.83-1.75 (m, 3H, H27a, H30a, H31a), 1.72-1.64 (m, 2H, H18a, H28a ), 1.57 (d, $J=$ $9.6 \mathrm{~Hz}, 3 \mathrm{H}, \mathrm{H} 26, \mathrm{H} 29), 1.49$ (d, $\left.J=6.7 \mathrm{~Hz}, 4 \mathrm{H}, \mathrm{H} 14 \mathrm{a}-\mathrm{CH}_{3}, \mathrm{H} 20 \mathrm{~b}\right), 1.36-1.13$ (m, 15H, H2'b, H27b, H28b, H30b, H31b, H2'b, H5'-Me, H5"-Me, H12a-CH $), 0.93$ (d, J = $\left.7.2 \mathrm{~Hz}, 3 \mathrm{H}, \mathrm{H} 24 \mathrm{a}-\mathrm{CH}_{3}\right), 0.89-0.79$ (m, 1H, H18b). ${ }^{13} \mathrm{C} \mathrm{NMR}\left(100 \mathrm{MHz}, \mathrm{CDCl}_{3}\right) \delta$ $173.5,164.0,161.7,159.2,153.7,139.5,138.0,137.8,136.1,135.0,132.0,127.7$, 125.1, 124.7, 120.4, 119.6, 118.2, 118.0, 116.6, 98.7, 95.7, 94.8, 81.7, 80.9, 80.3, 79.2, $73.8,68.3,68.2,68.2,67.7,67.1,65.4,56.6,56.1,52.5,49.9,45.7,41.1,40.3,39.7$, $38.6,36.6,34.6,34.3,31.8,31.4,30.0,26.9,26.6,26.5,25.5,20.2,19.9,18.3,17.3$, $16.6,15.1$.

S-3: The ${ }^{1} \mathrm{H}-\mathrm{NMR}$ and ${ }^{13} \mathrm{C}-\mathrm{NMR}$ data of 5c:

${ }^{1} \mathrm{H}$ NMR (400 MHz, $\left.\mathrm{CDCl}_{3}\right) \delta 8.71(\mathrm{~d}, J=9.9 \mathrm{~Hz}, 1 \mathrm{H}, \mathrm{CONHCO}), 8.47$ (s, 1H, CONH), 7.75-7.68 (m, 1H, Ph), 7.45 (dd, $J=3.9,1.3 \mathrm{~Hz}, 2 \mathrm{H}, \mathrm{Ph}), 7.37$ (ddd, $J=7.6$, 5.5, 3.2 Hz, 1H, Ph), 5.92-5.85 (m, 1H, H9), 5.83 -5.67 (m, 3H, H10, H11, H23), 5.54 (dd, $J=9.9,2.5 \mathrm{~Hz}, 1 \mathrm{H}, \mathrm{H} 22), 5.50-5.34$ (m, 3H, H3, H19, H1'), 5.00 (d, J=10.7 Hz, 1H, H15), 4.79 (d, $J=3.8$ Hz, 1H, H1'), 4.68 (t, $J=3.0$ Hz, 2H, H8a), 4.40-4.34 (m, 1H, H4"), 4.33-4.26 (m, 1H, H5), 4.15-4.06 (m, 2H, H5", H7-OH), 3.97 (d, J = 6.2 Hz, 1H, H6), 3.94 (s, 1H, H13), 3.91-3.79 (m, 2H, H17, H5), 3.73 (dt, J = 12.2, 4.4 Hz, 1H, H3"), 3.66-3.56 (m, 1H, H3'), 3.43 (s, 6H, H3'-OMe, H3"'-OMe), 3.34-3.20 
(m, 3H, H2, H25, H4), 2.53 (t, $J=7.3 \mathrm{~Hz}, 1 \mathrm{H}, \mathrm{H} 12), 2.38$ (d, $J=8.2 \mathrm{~Hz}, 1 \mathrm{H}$, H5-OH), 2.33-2.19 (m, 4H, H16, H24, H2"a), 2.10-1.94 (m, 2H, H2'a, H20a), 1.87 (s, 3H, H4a-CH ${ }_{3}$, 1.82-1.73 (m, 3H, H27a, H30a, H31a), 1.71-1.65 (m, 2H, H18a, H28a), $1.56(\mathrm{~d}, J=8.0 \mathrm{~Hz}, 3 \mathrm{H}, \mathrm{H} 26, \mathrm{H} 29), 1.48$ (d, $\left.J=10.3 \mathrm{~Hz}, 4 \mathrm{H}, \mathrm{H} 14 \mathrm{a}-\mathrm{CH}_{3}, \mathrm{H} 20 \mathrm{~b}\right)$, 1.35-1.11 (m, 15H, H2'b, H27b, H28b, H30b, H31b, H2'b, H5'-Me, H5"-Me, $\left.\mathrm{H} 12 \mathrm{a}-\mathrm{CH}_{3}\right), 0.93$ (d, $\left.J=7.2 \mathrm{~Hz}, 3 \mathrm{H}, \mathrm{H} 24 \mathrm{a}-\mathrm{CH}_{3}\right), 0.88-0.82$ (m, 1H, H18b). ${ }^{13} \mathrm{C}$ NMR $\left(100 \mathrm{MHz} \mathrm{CDCl}_{3}\right) \delta 173.5,167.4,154.0,139.4,138.0,137.8,136.1,135.0,133.3$, 132.3, 131.1, 130.5, 130.1, 127.7, 127.0, 124.7, 120.5, 118.2, 118.0, 98.6, 95.7, 94.9, $81.8,80.9,80.2,79.3,79.2,73.8,68.3,68.3,68.2,67.7,67.1,65.4,56.6,56.0,52.5$, 49.8, 45.7, 40.3, 39.7, 38.6, 36.6, 34.5, 34.3, 31.7, 31.4, 30.0, 26.9, 26.6, 26.4, 25.5, 20.2, 19.9, 18.3, 17.3, 16.6, 15.1 .

S-4: The ${ }^{1} \mathrm{H}-\mathrm{NMR}$ and ${ }^{13} \mathrm{C}-\mathrm{NMR}$ data of $\mathbf{5 d}$ :

${ }^{1} \mathrm{H}$ NMR (400 MHz, $\left.\mathrm{CDCl}_{3}\right) \delta 8.61(\mathrm{~d}, J=9.8 \mathrm{~Hz}, 1 \mathrm{H}, \mathrm{CONHCO}), 8.55$ (s, 1H, CONH), 7.75 (dd, $J=7.9,1.8 \mathrm{~Hz}, 1 \mathrm{H}, \mathrm{Ph}), 7.62$ (qd, $J=7.4,7.0,3.3 \mathrm{~Hz}, 3 \mathrm{H}, \mathrm{Ph}$ ), 5.93-5.86 (m, 1H, H9), 5.83-5.67 (m, 3H, H10, H11, H23), 5.54 (dd, J = 9.9, 2.5 Hz, 1H, H22), 5.49-5.33 (m, 3H, H3, H19, H1 "), 5.00 (d, J = 10.6 Hz, 1H, H15), 4.78 (d, $\left.J=3.7 \mathrm{~Hz}, 1 \mathrm{H}, \mathrm{H} 1^{\prime}\right), 4.67$ (t, $\left.J=2.9 \mathrm{~Hz}, 2 \mathrm{H}, \mathrm{H} 8 \mathrm{a}\right), 4.34-4.26$ (m, 2H, H4", H5), 4.17 (s, 1H, H7-OH), 4.12-4.06 (m, 1H, H5"), 3.97 (d, J = 6.2 Hz, 1H, H6), 3.94 (s, 1H, H13), 3.92-3.77 (m, 2H, H17, H5'), 3.71 (dt, $\left.J=12.2,4.4 \mathrm{~Hz}, 1 \mathrm{H}, \mathrm{H} 3^{\prime \prime}\right), 3.61$ (ddd, $J$ = 11.1, 8.4, 4.7 Hz, 1H, H3'), 3.42 (s, 3H, H3'-OMe), 3.38 (s, 3H, H3"'-OMe), 3.35-3.17 (m, 3H, H2, H25, H4), 2.53 (t, $J=7.3 \mathrm{~Hz}, 1 \mathrm{H}, \mathrm{H} 12), 2.41-2.18$ (m, 5H, H5-OH, H16, H24, H2"'a), 2.10-1.94 (m, 2H, H2'a, H20a), 1.86 (s, 3H, H4a-CH ${ }_{3}$ ), 1.83-1.72 (m, 3H, H27a, H30a, H31a), 1.72-1.65 (m, 2H, H18a, H28a), 1.56 (d, $J=$ $8.2 \mathrm{~Hz}, 3 \mathrm{H}, \mathrm{H} 26, \mathrm{H} 29), 1.47$ (d, $J=15.2 \mathrm{~Hz}, 4 \mathrm{H}, \mathrm{H} 14 \mathrm{a}-\mathrm{CH}_{3}, \mathrm{H} 20 \mathrm{~b}$ ), 1.37-1.10 (m, 15H, H2'"b, H27b, H28b, H30b, H31b, H2'b, H5'-Me, H5"-Me, H12a-CH ) $^{\prime} 0.93$ (d, $\left.J=7.1 \mathrm{~Hz}, 3 \mathrm{H}, \mathrm{H} 24 \mathrm{a}-\mathrm{CH}_{3}\right), 0.85(\mathrm{~d}, J=12.1 \mathrm{~Hz}, 1 \mathrm{H}, \mathrm{H} 18 \mathrm{~b}) .{ }^{13} \mathrm{C} \mathrm{NMR}(100 \mathrm{MHz}$, $\left.\mathrm{CDCl}_{3}\right) \delta 173.6,168.7,154.1,139.4,138.0,137.8,136.1,135.1,133.7,131.9,130.7$, 128.2, 127.8, 126.7, 124.7, 121.9, 120.5, 118.2, 117.9, 98.6, 95.7, 94.9, 81.8, 80.9, 80.2, 79.3, 79.2, 73.8, 68.4, 68.2, 68.2, 67.7, 67.1, 65.3, 56.6, 55.9, 52.5, 49.8, 45.8, $41.1,40.3,39.7,38.7,36.6,34.5,34.3,31.7,31.4,30.0,26.9,26.6,26.4,25.6,20.2$, 
$19.8,18.3,17.2,16.6,15.1$.

S-5: The ${ }^{1} \mathrm{H}-\mathrm{NMR}$ and ${ }^{13} \mathrm{C}-\mathrm{NMR}$ data of 5e:

${ }^{1} \mathrm{H}$ NMR (400 MHz, $\mathrm{CDCl}_{3}$ ) $\delta 8.97(\mathrm{~d}, J=9.9 \mathrm{~Hz}, 1 \mathrm{H}, \mathrm{CONHCO}), 8.47$ (s, 1H, CONH), 7.69 (s, 1H, Ph), 7.67-7.61 (m, 1H, Ph), 7.45-7.32 (m, 2H, Ph), 5.92-5.87 (m, 1H, H9), 5.83-5.67 (m, 3H, H10, H11, H23), 5.54 (dd, $J=9.8,2.5 \mathrm{~Hz}, 1 \mathrm{H}, \mathrm{H} 22$ ), 5.50-5.36 (m, 3H, H3, H19, H1 "), 5.01 (d, $J=10.6 \mathrm{~Hz}, 1 \mathrm{H}, \mathrm{H} 15), 4.79$ (d, $J=3.7 \mathrm{~Hz}$, 1H, H1'), 4.74-4.62 (m, 2H, H8a), 4.42-4.35 (m, 1H, H4"), 4.33-4.26 (m, 1H, H5), 4.16-4.07 (m, 2H, H5", H7-OH), 3.98 (d, $J=6.2 \mathrm{~Hz}, 1 \mathrm{H}, \mathrm{H} 6), 3.94$ (s, 1H, H13), 3.91-3.80 (m, 2H, H17, H5'), 3.74 (dt, $\left.J=12.2,4.4 \mathrm{~Hz}, 1 \mathrm{H}, \mathrm{H} 3{ }^{\prime \prime}\right), 3.61$ (ddd, $J=11.0$, 8.6, 4.7 Hz, 1H, H3'), 3.43 (d, $J=0.9 \mathrm{~Hz}, 6 \mathrm{H}, \mathrm{H} 3$ '-OMe, H3"'-OMe), 3.34-3.22 (m, 3H, H2, H25, H4'), 2.59-2.49 (m, 1H, H12), 2.41 (s, 3H, $\left.\mathrm{CH}_{3}\right), 2.38$ (d, J=8.2 Hz, 1H, H5-OH), 2.34-2.18 (m, 4H, H16, H24, H2"a), 2.11-1.97 (m, 2H, H2'a, H20a), 1.87 (s, 3H, H4a-CH $), 1.83-1.73$ (m, 3H, H27a, H30a, H31a), 1.72-1.67 (m, 2H, H18a, H28a), $1.55(\mathrm{~d}, J=3.7 \mathrm{~Hz}, 3 \mathrm{H}, \mathrm{H} 26, \mathrm{H} 29), 1.49$ (d, $J=7.6 \mathrm{~Hz}, 4 \mathrm{H}, \mathrm{H} 14 \mathrm{a}-\mathrm{CH}_{3}, \mathrm{H} 20 \mathrm{~b}$ ), 1.34-1.12 (m, 15H, H2'b, H27b, H28b, H30b, H31b, H2'b, H5'-Me, H5"-Me, $\left.\mathrm{H} 12 \mathrm{a}-\mathrm{CH}_{3}\right), 0.93$ (d, $\left.J=7.1 \mathrm{~Hz}, 3 \mathrm{H}, \mathrm{H} 24 \mathrm{a}-\mathrm{CH}_{3}\right), 0.88-0.82(\mathrm{~m}, 1 \mathrm{H}, \mathrm{H} 18 \mathrm{~b}) .{ }^{13} \mathrm{C}$ NMR $\left(100 \mathrm{MHz}, \mathrm{CDCl}_{3}\right) \delta 173.5,168.3,154.8,139.5,138.6,138.0,137.8,136.1,135.0$, $133.7,132.4,128.5,128.4,127.7,124.7,124.7,120.5,118.2,118.1,98.7,95.7,94.9$, $81.8,81.0,80.4,79.2,73.9,68.3,68.3,68.2,67.7,67.1,65.5,56.6,56.0,52.5,49.9$, $45.7,41.1,40.4,39.7,38.6,36.6,34.6,34.3,31.7,31.4,30.0,26.9,26.6,26.5,25.5$, $21.3,20.2,19.9,18.3,17.3,16.6,15.1$.

S-6: The ${ }^{1} \mathrm{H}-\mathrm{NMR}$ and ${ }^{13} \mathrm{C}-\mathrm{NMR}$ data of $\mathbf{5 f}$ :

${ }^{1} \mathrm{H}$ NMR (400 MHz, $\mathrm{CDCl}_{3}$ ) $\delta 9.69$ (s, 1H, CONHCO), 9.03 (d, $J=9.9 \mathrm{~Hz}, 1 \mathrm{H}$, CONH), 8.31 (s, 1H, Ph), 8.15 (d, J=7.9 Hz, 1H, pH), 7.84 (d, J= 7.8 Hz, 1H, Ph), 7.63 (t, $J=7.8 \mathrm{~Hz}, 1 \mathrm{H}, \mathrm{Ph}$ ), 5.90 (d, $J=9.8 \mathrm{~Hz}, 1 \mathrm{H}, \mathrm{H} 9), 5.84-5.67$ (m, 3H, H10, H11, H23), 5.54 (dd, $J=9.9,2.5 \mathrm{~Hz}, 1 \mathrm{H}, \mathrm{H} 22), 5.50-5.35$ (m, 3H, H3, H19, H1 "), 5.01 (d, $J=10.9 \mathrm{~Hz}, 1 \mathrm{H}, \mathrm{H} 15), 4.79$ (d, $\left.J=3.8 \mathrm{~Hz}, 1 \mathrm{H}, \mathrm{H1} 1^{\prime}\right), 4.69$ (t, $\left.J=2.9 \mathrm{~Hz}, 2 \mathrm{H}, \mathrm{H} 8 \mathrm{a}\right)$, 4.41 (d, $\left.J=10.5 \mathrm{~Hz}, 1 \mathrm{H}, \mathrm{H} 4{ }^{\prime \prime}\right), 4.30$ (d, $\left.J=6.3 \mathrm{~Hz}, 1 \mathrm{H}, \mathrm{H} 5\right), 4.18-4.05$ (m, 2H, H5", H7-OH), 3.98 (d, J = 6.2 Hz, 1H, H6), 3.95 (s, 1H, H13), 3.91-3.80 (m, 2H, H17, H5'), 3.74 (dt, $\left.J=12.1,4.4 \mathrm{~Hz}, 1 \mathrm{H}, \mathrm{H} 3^{\prime \prime}\right), 3.66-3.57$ (m, 1H, H3'), 3.43 (s, 3H, H3'-OMe), 
3.36 (s, 3H, H3"'-OMe), 3.34-3.20 (m, 3H, H2, H25, H4'), 2.53 (t, $J=7.3 \mathrm{~Hz}, 1 \mathrm{H}$, H12), 2.40-2.18 (m, 5H, H5-OH, H16, H24, H2"a), 2.11-1.96 (m, 2H, H2'a, H20a), 1.88 (s, 3H, H4a-CH $), 1.82-1.73$ (m, 3H, H27a, H30a, H31a), 1.73-1.63 (m, 2H, H18a, H28a), 1.58-1.53 (m, 3H, H26, H29), 1.49 (d, $J=7.8 \mathrm{~Hz}, 4 \mathrm{H}, \mathrm{H} 14 \mathrm{a}-\mathrm{CH}_{3}$, H20b), 1.36-1.13 (m, 15H, H2'b, H27b, H28b, H30b, H31b, H2'b, H5'-Me, H5"-Me, $\left.\mathrm{H} 12 \mathrm{a}-\mathrm{CH}_{3}\right), 0.93\left(\mathrm{~d}, J=7.1 \mathrm{~Hz}, 3 \mathrm{H}, \mathrm{H} 24 \mathrm{a}-\mathrm{CH}_{3}\right), 0.86(\mathrm{~d}, J=12.6 \mathrm{~Hz}, 1 \mathrm{H}, \mathrm{H} 18 \mathrm{~b}) .{ }^{13} \mathrm{C}$ NMR (100 MHz, $\left.\mathrm{CDCl}_{3}\right) \delta 173.6,167.2,155.4,139.5,138.0,137.8,136.1,135.1$, $133.5,131.7,131.1,130.8,129.1,127.7,125.1,124.7,122.3,120.5,118.2,118.1$, 98.8, 95.7, 94.9, 81.8, 81.1, 80.4, 79.2, 73.8, 68.3, 68.3, 68.2, 67.7, 67.1, 65.4, 56.6, 55.9, 52.5, 49.8, 45.7, 41.1, 40.3, 39.7, 38.6, 36.6, 34.6, 34.3, 31.8, 31.4, 30.0, 26.9, 26.6, 26.5, 25.5, 20.2, 19.9, 18.3, 17.2, 16.6, 15.1 .

S-7: The ${ }^{1} \mathrm{H}-\mathrm{NMR}$ and ${ }^{13} \mathrm{C}-\mathrm{NMR}$ data of $\mathbf{5 g}$ :

${ }^{1} \mathrm{H}$ NMR (400 MHz, $\left.\mathrm{CDCl}_{3}\right) \delta 8.97(\mathrm{~d}, J=9.8 \mathrm{~Hz}, 1 \mathrm{H}, \mathrm{CONHCO}), 8.82(\mathrm{~s}, 1 \mathrm{H}$, CONH), 7.97-7.90 (m, 2H, Ph), 7.16 (t, $J=8.6 \mathrm{~Hz}, 2 \mathrm{H}, \mathrm{Ph}), 5.90(\mathrm{~d}, J=9.7 \mathrm{~Hz}, 1 \mathrm{H}$, H9), 5.82-5.68 (m, 3H, H10, H11, H23), 5.54 (dd, $J=9.9,2.5 \mathrm{~Hz}, 1 \mathrm{H}, \mathrm{H} 22)$, 5.50-5.33 (m, 3H, H3, H19, H1 "), 5.01 (d, $J=10.7 \mathrm{~Hz}, 1 \mathrm{H}, \mathrm{H} 15), 4.79$ (d, $J=3.8 \mathrm{~Hz}$, 1H, H1'), 4.69 (t, $J=3.0 \mathrm{~Hz}, 2 \mathrm{H}, \mathrm{H} 8 \mathrm{a}), 4.37$ (d, $\left.J=9.8 \mathrm{~Hz}, 1 \mathrm{H}, \mathrm{H} 4{ }^{\prime \prime}\right), 4.30$ (d, $J=6.2$ Hz, 1H, H5), 4.17-4.07 (m, 2H, H5", H7-OH), 3.98 (d, J = 6.2 Hz, 1H, H6), 3.95 (s, 1H, H13), 3.92-3.80 (m, 2H, H17, H5'), 3.74 (dt, $\left.J=12.1,4.3 \mathrm{~Hz}, 1 \mathrm{H}, \mathrm{H} 3{ }^{\prime \prime}\right), 3.61$ (td, $J=10.6,8.8,4.8 \mathrm{~Hz}, 1 \mathrm{H}, \mathrm{H} 3$ ), 3.43 (s, 3H, H3'-OMe), 3.42 (s, 3H, H3"'-OMe), 3.35-3.22 (m, 3H, H2, H25, H4'), 2.58-2.48 (m, 1H, H12), 2.39-2.19 (m, 5H, H5-OH, H16, H24, H2"a), 2.11-1.97 (m, 2H, H2'a, H20a), 1.87 (s, 3H, H4a-CH ) $^{\prime}$ 1.82-1.74 (m, 3H, H27a, H30a, H31a), 1.73-1.64 (m, 2H, H18a, H28a), 1.56 (d, J = 8.6 Hz, 3H, H26, H29), 1.49 (d, $J=7.8 \mathrm{~Hz}, 4 \mathrm{H}, \mathrm{H} 14 \mathrm{a}-\mathrm{CH}_{3}, \mathrm{H} 20 \mathrm{~b}$ ), 1.30-1.15 (m, 15H, H2' b, H27b, H28b, H30b, H31b, H2 b, H5'-Me, H5"-Me, H12a-CH ${ }_{3}$ ), 0.93 (d, J = 7.2 Hz, $\left.3 \mathrm{H}, \mathrm{H} 24 \mathrm{a}-\mathrm{CH}_{3}\right), 0.86(\mathrm{~d}, J=12.8 \mathrm{~Hz}, 1 \mathrm{H}, \mathrm{H} 18 \mathrm{~b}) .{ }^{13} \mathrm{C} \mathrm{NMR}\left(100 \mathrm{MHz}, \mathrm{CDCl}_{3}\right) \delta$ 173.6, 167.2, 164.3, 155.0, 139.5, 138.0, 137.8, 136.2, 135.1, 130.6, 130.5, 128.8, 127.7, 124.7, 120.5, 118.2, 118.1, 115.8, 115.6, 98.7, 95.7, 94.9, 81.8, 81.1, 80.4, 79.2, 73.9, 68.3, 68.2, 67.7, 67.1, 65.4, 56.6, 56.0, 52.5, 49.9, 45.7, 41.1, 40.4, 39.7, 38.6, $36.6,34.6,34.3,31.7,31.4,30.0,29.7,26.9,26.6,26.5,25.5,20.2,19.9,18.3,17.3$, 
16.6, 15.1.

S-8: The ${ }^{1} \mathrm{H}-\mathrm{NMR}$ and ${ }^{13} \mathrm{C}-\mathrm{NMR}$ data of $\mathbf{5 h}$ :

${ }^{1} \mathrm{H}$ NMR (400 MHz, $\left.\mathrm{CDCl}_{3}\right) \delta 8.95(\mathrm{~d}, J=9.9 \mathrm{~Hz}, 1 \mathrm{H}, \mathrm{CONHCO}), 8.88(\mathrm{~s}, 1 \mathrm{H}$, CONH), 7.86 (d, $J=8.6 \mathrm{~Hz}, 2 \mathrm{H}, \mathrm{Ph}), 7.46$ (d, $J=8.6 \mathrm{~Hz}, 2 \mathrm{H}, \mathrm{Ph}), 5.93-5.85$ (m, 1H, H9), 5.84-5.66 (m, 3H, H10, H11, H23), 5.54 (dd, $J=9.9,2.6 \mathrm{~Hz}, 1 \mathrm{H}, \mathrm{H} 22$ ), 5.50-5.34 (m, 3H, H3, H19, H1 "), 5.01 (d, $J=10.8 \mathrm{~Hz}, 1 \mathrm{H}, \mathrm{H} 15), 4.79$ (d, $J=3.8 \mathrm{~Hz}$, 1H, H1'), 4.75-4.61 (m, 2H, H8a), 4.37 (dd, $\left.J=10.0,3.5 \mathrm{~Hz}, 1 \mathrm{H}, \mathrm{H} 4{ }^{\prime \prime}\right), 4.30$ (d, $J=$ $6.2 \mathrm{~Hz}, 1 \mathrm{H}, \mathrm{H} 5), 4.18-4.05$ (m, 2H, H5", H7-OH), 3.98 (d, J=6.2 Hz, 1H, H6), 3.95 (s, 1H, H13), 3.92-3.81 (m, 2H, H17, H5'), 3.75 (dt, $J=12.2,4.4$ Hz, 1H, H3"), 3.61 (ddd, $J=11.2,8.6,4.6 \mathrm{~Hz}, 1 \mathrm{H}, \mathrm{H} 3$ '), 3.43 (s, 3H, H3'-OMe), 3.42 (s, 3H, H3"'-OMe), 3.34-3.22 (m, 3H, H2, H25, H4), 2.53 (t, $J=7.7 \mathrm{~Hz}, 1 \mathrm{H}, \mathrm{H} 12), 2.40-2.18$ (m, 5H, H5-OH, H16, H24, H2"a), 2.12-1.97 (m, 2H, H2'a, H20a), 1.87 (s, 3H, H4a-CH ${ }_{3}$ ), 1.84-1.73 (m, 3H, H27a, H30a, H31a), 1.72-1.64 (m, 2H, H18a, H28a), 1.57 (d, $J=$ $9.0 \mathrm{~Hz}, 3 \mathrm{H}, \mathrm{H} 26, \mathrm{H} 29), 1.49$ (d, $J=7.7 \mathrm{~Hz}, 4 \mathrm{H}, \mathrm{H} 14 \mathrm{a}-\mathrm{CH}_{3}, \mathrm{H} 20 \mathrm{~b}$ ), $1.36-1.13$ (m, 15H, H2'b, H27b, H28b, H30b, H31b, H2 b, H5'-Me, H5"'-Me, H12a-CH ${ }_{3}$ ), 0.93 (d, $\left.J=7.1 \mathrm{~Hz}, 3 \mathrm{H}, \mathrm{H} 24 \mathrm{a}-\mathrm{CH}_{3}\right), 0.89-0.82(\mathrm{~m}, 1 \mathrm{H}, \mathrm{H} 18 \mathrm{~b}) .{ }^{13} \mathrm{C} \mathrm{NMR}\left(100 \mathrm{MHz}, \mathrm{CDCl}_{3}\right) \delta$ 173.6, 167.4, 155.1, 139.5, 139.3, 138.0, 137.8, 136.2, 135.1, 131.0, 129.5(2-C), 128.9(2-C), 127.7, 124.7, 120.5, 118.2, 118.0, 98.7, 95.7, 94.9, 81.8, 81.1, 80.3, 79.3, 79.2, 73.9, 68.4, 68.2, 67.7, 67.1, 65.4, 56.6, 56.0, 52.5, 50.0, 45.8, 40.4, 39.7, 38.6, $36.6,34.6,34.3,31.7,31.4,30.0,26.9,26.6,26.4,25.5,20.2,19.9,18.3,17.3,16.6$, 15.1.

S-9: The ${ }^{1} \mathrm{H}-\mathrm{NMR}$ and ${ }^{13} \mathrm{C}-\mathrm{NMR}$ data of 5i:

${ }^{1} \mathrm{H}$ NMR (400 MHz, $\mathrm{CDCl}_{3}$ ) $\delta 9.23(\mathrm{~s}, 1 \mathrm{H}, \mathrm{CONHCO}), 9.00$ (d, $J=9.8 \mathrm{~Hz}, 1 \mathrm{H}$, CONH), $7.82(\mathrm{~d}, J=8.6 \mathrm{~Hz}, 2 \mathrm{H}, \mathrm{Ph}), 7.61(\mathrm{~d}, J=8.6 \mathrm{~Hz}, 2 \mathrm{H}, \mathrm{Ph}), 5.93-5.87(\mathrm{~m}, 1 \mathrm{H}$, H9), 5.83-5.68 (m, 3H, H10, H11, H23), 5.54 (dd, $J=9.9,2.5 \mathrm{~Hz}, 1 \mathrm{H}, \mathrm{H} 22)$, 5.49-5.35 (m, 3H, H3, H19, H1"), 5.01 (d, $J=10.9$ Hz, 1H, H15), 4.79 (d, J=3.8 Hz, 1H, H1'), 4.74-4.63 (m, 2H, H8a), 4.39-4.33 (m, 1H, H4"), 4.30 (t, J = 7.2 Hz, 1H, H5), 4.17-4.08 (m, 2H, H5", H7-OH), 3.97 (d, J = 6.2 Hz, 1H, H6), 3.94 (s, 1H, H13), 3.91-3.80 (m, 2H, H17, H5'), 3.75 (dt, $J=12.2,4.4$ Hz, 1H, H3'), 3.61 (ddd, $J=11.3$, 8.6, 4.8 Hz, 1H, H3'), 3.43 (s, 3H, H3'-OMe), 3.40 (s, 3H, H3"'-OMe), 3.35-3.21 (m, 
3H, H2, H25, H4'), 2.53 (t, $J=7.8 \mathrm{~Hz}, 1 \mathrm{H}, \mathrm{H} 12), 2.38$ (d, $J=8.2 \mathrm{~Hz}, 1 \mathrm{H}, \mathrm{H} 5-\mathrm{OH})$, 2.35-2.20 (m, 4H, H16, H24, H2"a), 2.10-1.97 (m, 2H, H2'a, H20a), 1.87 (s, 3H, H4a-CH $_{3}$, 1.82-1.74 (m, 3H, H27a, H30a, H31a), 1.73-1.67 (m, 2H, H18a, H28a), 1.59-1.53 (m, 3H, H26, H29), 1.49 (d, $J=8.6 \mathrm{~Hz}, 4 \mathrm{H}, \mathrm{H} 14 \mathrm{a}-\mathrm{CH}_{3}, \mathrm{H} 20 \mathrm{~b}$ ), 1.35-1.12 (m, 15H, H2'"b, H27b, H28b, H30b, H31b, H2'b, H5'-Me, H5"-Me, H12a-CH $), 0.93$ $\left(\mathrm{d}, J=7.1 \mathrm{~Hz}, 3 \mathrm{H}, \mathrm{H} 24 \mathrm{a}-\mathrm{CH}_{3}\right), 0.86$ (d, $\left.J=12.2 \mathrm{~Hz}, 1 \mathrm{H}, \mathrm{H} 18 \mathrm{~b}\right) .{ }^{13} \mathrm{C}$ NMR $(100 \mathrm{MHz}$, $\left.\mathrm{CDCl}_{3}\right) \delta 173.5,167.6,155.1,139.4,138.0,137.7,136.2,135.1,131.8(2-\mathrm{C}), 131.4$, 129.7(2-C), 127.9, 127.7, 124.7, 120.5, 118.2, 118.0, 98.7, 95.7, 94.9, 81.8, 81.1, 80.3, $79.3,79.2,73.8,68.4,68.3,68.2,67.7,67.1,65.4,56.6,56.0,52.5,50.0,45.8,40.45$ 39.7, 38.6, 36.6, 34.6, 34.3, 31.7, 31.4, 30.0, 26.9, 26.6, 26.4, 25.5, 20.2, 19.9, 18.3, $17.3,16.6,15.1$.

S-10: The ${ }^{1} \mathrm{H}-\mathrm{NMR}$ and ${ }^{13} \mathrm{C}-\mathrm{NMR}$ data of $\mathbf{5 j}$ :

${ }^{1} \mathrm{H}$ NMR $\left(400 \mathrm{MHz}, \mathrm{CDCl}_{3}\right) \delta 8.98(\mathrm{~d}, J=9.9 \mathrm{~Hz}, 1 \mathrm{H}, \mathrm{CONHCO}), 8.27(\mathrm{~s}, 1 \mathrm{H}$, CONH), $7.83(\mathrm{~d}, J=8.9 \mathrm{~Hz}, 2 \mathrm{H}, \mathrm{Ph}), 6.96$ (d, $J=8.9 \mathrm{~Hz}, 2 \mathrm{H}, \mathrm{Ph}), 5.90$ (d, $J=9.9 \mathrm{~Hz}$, 1H, H9), 5.84-5.66 (m, 3H, H10, H11, H23), 5.54 (dd, $J=9.8,2.5 \mathrm{~Hz}, 1 \mathrm{H}, \mathrm{H} 22$ ), 5.50-5.31 (m, 3H, H3, H19, H1'), 5.01 (d, $J=10.8$ Hz, 1H, H15), 4.81-4.77 (m, 1H, H1'), 4.69 (t, $J=2.9 \mathrm{~Hz}, 2 \mathrm{H}, \mathrm{H} 8 \mathrm{a}), 4.38$ (d, $\left.J=9.4 \mathrm{~Hz}, 1 \mathrm{H}, \mathrm{H} 4^{\prime \prime}\right), 4.30$ (t, $J=7.2 \mathrm{~Hz}$, 1H, H5), 4.16-4.08 (m, 1H, H5"), 4.07 (s, 1H, H7-OH), 3.97 (d, J = 6.2 Hz, 1H, H6), 3.94 (s, 1H, H13), 3.92-3.79 (s, 2H, H17, H5'), 3.87 (s, 3H, $\left.\mathrm{OCH}_{3}\right), 3.74$ (dt, $J=12.2$, $\left.4.4 \mathrm{~Hz}, 1 \mathrm{H}, \mathrm{H} 3^{\prime \prime}\right), 3.61$ (td, $J=10.1,8.7,4.6 \mathrm{~Hz}, 1 \mathrm{H}, \mathrm{H} 3$ '), 3.44 (s, 3H, H3'-OMe), 3.43 (s, 3H, H3"--OMe), 3.36-3.20 (m, 3H, H2, H25, H4'), 2.53 (t, $J=7.4 \mathrm{~Hz}, 1 \mathrm{H}$, H12), 2.37 (d, $J=8.2 \mathrm{~Hz}, 1 \mathrm{H}, \mathrm{H} 5-\mathrm{OH}), 2.34-2.19$ (m, 4H, H16, H24, H2"a), 2.10-1.97 (m, 2H, H2' a, H20a), 1.87 (t, $\left.J=2.0 \mathrm{~Hz}, 3 \mathrm{H}, \mathrm{H} 4 \mathrm{a}-\mathrm{CH}_{3}\right), 1.84-1.75$ (m, 3H, H27a, H30a, H31a), 1.73-1.64 (m, 2H, H18a, H28a), 1.56 (d, J = 11.7 Hz, 3H, H26, H29), 1.49 (d, $\left.J=7.6 \mathrm{~Hz}, 4 \mathrm{H}, \mathrm{H} 14 \mathrm{a}-\mathrm{CH}_{3}, \mathrm{H} 20 \mathrm{~b}\right), 1.36-1.12$ (m, 15H, H2'b, H27b, H28b, H30b, H31b, H2'b, H5'-Me, H5"-Me, H12a-CH $), 0.93$ (d, J = $7.1 \mathrm{~Hz}, 3 \mathrm{H}$, $\left.\mathrm{H} 24 \mathrm{a}-\mathrm{CH}_{3}\right), 0.88-0.82$ (m, 1H, H18b). ${ }^{13} \mathrm{C} \mathrm{NMR}\left(100 \mathrm{MHz}, \mathrm{CDCl}_{3}\right) \delta 173.5,167.5$, 163.3, 155.0, 139.5, 138.0, 137.8, 136.2, 135.0, 129.8(2-C), 127.7, 124.7(2-C), 120.5, 118.2, 118.1, 113.9, 98.7, 95.7, 94.9, 81.8, 81.0, 80.4, 79.2, 73.9, 68.4, 68.3, 68.2, $67.7,67.1,65.5,56.7,56.0,55.5,52.5,49.8,45.7,41.1,40.4,39.7,38.6,36.6,34.6$, 
34.3, 31.8, 31.4, 30.0, 29.6, 26.9, 26.6, 26.5, 25.5, 20.2, 19.9, 18.3, 17.3, 16.6, 15.1.

S-11: The ${ }^{1} \mathrm{H}-\mathrm{NMR}$ and ${ }^{13} \mathrm{C}-\mathrm{NMR}$ data of $5 \mathbf{k}$ :

${ }^{1} \mathrm{H}$ NMR (400 MHz, $\mathrm{CDCl}_{3}$ ) $\delta 9.37$ (s, 1H, CONHCO), 8.97 (d, $J=9.8 \mathrm{~Hz}, 1 \mathrm{H}$, $\mathrm{CONH}), 8.07$ (d, $J=8.1 \mathrm{~Hz}, 2 \mathrm{H}, \mathrm{Ph}), 7.74(\mathrm{~d}, J=8.3 \mathrm{~Hz}, 2 \mathrm{H}, \mathrm{Ph}), 5.93-5.87$ (m, 1H, H9), 5.84-5.68 (m, 3H, H10, H11, H23), 5.54 (dd, $J=9.9,2.6 \mathrm{~Hz}, 1 \mathrm{H}, \mathrm{H} 22$ ), 5.50-5.37 (m, 3H, H3, H19, H1 "), 5.01 (d, $J=10.9 \mathrm{~Hz}, 1 \mathrm{H}, \mathrm{H} 15), 4.79$ (d, $J=3.2 \mathrm{~Hz}$, 1H, H1'), 4.74-4.62 (m, 2H, H8a), 4.36 (dd, $\left.J=10.1,3.4 \mathrm{~Hz}, 1 \mathrm{H}, \mathrm{H} 4{ }^{\prime \prime}\right), 4.30$ (t, $J=$ 7.2 Hz, 1H, H5), 4.19-4.08 (m, 2H, H5", H7-OH), 3.98 (d, J=6.2 Hz, 1H, H6), 3.95 (s, 1H, H13), 3.92-3.81 (m, 2H, H17, H5'), 3.75 (dt, $J=12.2,4.4$ Hz, 1H, H3'), 3.62 (ddd, $J=11.2,8.5,4.7 \mathrm{~Hz}, 1 \mathrm{H}, \mathrm{H} 3$ '), 3.43 (s, 3H, H3'-OMe), 3.39 (s, 3H, H3'--OMe), 3.35-3.20 (m, 3H, H2, H25, H4), 2.53 (t, $J=7.2 \mathrm{~Hz}, 1 \mathrm{H}, \mathrm{H} 12), 2.36$ (d, $J=8.2 \mathrm{~Hz}$, 1H, H5-OH), 2.33-2.20 (m, 4H, H16, H24, H2"a), 2.13-1.97 (m, 2H, H2'a, H20a), 1.87 (s, 3H, H4a-CH $), 1.83-1.74$ (m, 3H, H27a, H30a, H31a), 1.73-1.64 (m, 2H, H18a, H28a), 1.57 (d, $J=8.6$ Hz, 3H, H26, H29), 1.49 (d, $J=8.4$ Hz, 4H, H14a-CH , H20b), 1.39-1.13 (m, 15H, H2'"b, H27b, H28b, H30b, H31b, H2'b, H5'-Me, H5 "'-Me, $\left.\mathrm{H} 12 \mathrm{a}-\mathrm{CH}_{3}\right), 0.93$ (d, $\left.J=7.1 \mathrm{~Hz}, 3 \mathrm{H}, \mathrm{H} 24 \mathrm{a}-\mathrm{CH}_{3}\right), 0.86$ (d, $\left.J=12.4 \mathrm{~Hz}, 1 \mathrm{H}, \mathrm{H} 18 \mathrm{~b}\right) .{ }^{13} \mathrm{C}$ NMR $\left(100 \mathrm{MHz}, \mathrm{CDCl}_{3}\right) \delta 173.6,167.3,155.0,139.4,138.0,137.8,136.2,135.9$, 135.1, 134.4, 134.1, 128.6(2-C), 127.7, 125.6, 125.5, 124.8, 124.7, 122.1, 120.5, 118.2, 118.0, 98.7, 95.7, 94.9, 81.8, 81.1, 80.3, 79.2, 73.9, 68.4, 68.2, 67.7, 67.1, 65.3, 56.6, 55.9, 52.5, 50.1, 45.8, 40.4, 39.7, 38.6, 36.6, 34.6, 34.3, 31.7, 31.4, 30.0, 26.9, $26.6,26.5,25.5,20.2,19.9,18.3,17.3,16.6,15.1$.

S-12: The ${ }^{1} \mathrm{H}-\mathrm{NMR}$ and ${ }^{13} \mathrm{C}-\mathrm{NMR}$ data of 5l:

${ }^{1} \mathrm{H}$ NMR (400 MHz, $\left.\mathrm{CDCl}_{3}\right) \delta 9.21(\mathrm{~s}, 1 \mathrm{H}, \mathrm{CONHCO}), 8.74(\mathrm{~d}, J=9.8 \mathrm{~Hz}, 1 \mathrm{H}$, CONH), 5.93-5.86 (m, 1H, H9), 5.82-5.67 (m, 3H, H10, H11, H23), 5.54 (dd, J = 9.9, $2.5 \mathrm{~Hz}, 1 \mathrm{H}, \mathrm{H} 22), 5.46-5.35$ (m, 3H, H3, H19, H1"), 5.04-4.97 (m, 1H, H15), 4.80-4.76 (m, 1H, H1'), 4.72-4.63 (m, 2H, H8a), 4.35- 4.25 (m, 2H, H4", H5), 4.13 (s, 1H, H7-OH), 4.10-4.02 (m, 1H, H5"), 3.97 (d, J = 6.2 Hz, 1H, H6), 3.93 (s, 1H, H13), 3.89-3.77 (m, 2H, H17, H5'), 3.69 (dt, $\left.J=12.2,4.3 \mathrm{~Hz}, 1 \mathrm{H}, \mathrm{H} 3{ }^{\prime \prime}\right), 3.60$ (td, $J=10.1$, 9.2, 4.9 Hz, 1H, H3'), 3.41 (d, $J=3.1 \mathrm{~Hz}, 6 \mathrm{H}, \mathrm{H} 3$ '-OMe, H3"'-OMe), 3.33-3.19 (m, 3H, H2, H25, H4), 2.57-2.47 (m, 1H, H12), 2.42-2.19 (m, 5H, H5-OH, H16, H24, 
$\mathrm{H} 2$ 'a), 2.12 (d, $\left.J=2.8 \mathrm{~Hz}, 3 \mathrm{H}, \mathrm{COCH}_{3}\right), 2.01$ (dt, $\left.J=11.0,3.9 \mathrm{~Hz}, 2 \mathrm{H}, \mathrm{H} 2^{\prime} \mathrm{a}, \mathrm{H} 20 \mathrm{a}\right)$, 1.87 (s, 3H, H4a-CH $)$, 1.83-1.74 (m, 3H, H27a, H30a, H31a), 1.73-1.64 (m, 2H, H18a, H28a), 1.56 (d, $J=8.1 \mathrm{~Hz}, 3 \mathrm{H}, \mathrm{H} 26, \mathrm{H} 29), 1.48$ (d, $J=7.8 \mathrm{~Hz}, 4 \mathrm{H}, \mathrm{H} 14 \mathrm{a}-\mathrm{CH}_{3}$, H20b), 1.36-1.12 (m, 15H, H2'b, H27b, H28b, H30b, H31b, H2'b, H5'-Me, H5"-Me, $\left.\mathrm{H} 12 \mathrm{a}-\mathrm{CH}_{3}\right), 0.92$ (d, $\left.J=7.1 \mathrm{~Hz}, 3 \mathrm{H}, \mathrm{H} 24 \mathrm{a}-\mathrm{CH}_{3}\right), 0.85$ (d, $\left.J=12.0 \mathrm{~Hz}, 1 \mathrm{H}, \mathrm{H} 18 \mathrm{~b}\right) .{ }^{13} \mathrm{C}$ NMR (100 MHz, $\left.\mathrm{CDCl}_{3}\right) \delta 173.6,172.3,155.3,139.4,138.0,137.8,136.1,135.0$, $127.7,124.7,120.5,118.2,118.0,98.6,95.6,94.9,81.8,80.7,80.3,79.3,79.2,73.9$, 68.3, 68.2, 67.7, 67.1, 65.3, 56.6, 56.0, 52.5, 49.5, 45.7, 41.1, 40.3, 39.7, 38.6, 36.6, 34.6, 34.3, 31.6, 31.4, 30.0, 26.9, 26.6, 26.4, 25.5, 23.9, 20.2, 19.9, 18.2, 17.2, 16.6, 15.1 .

S-13: The ${ }^{1} \mathrm{H}-\mathrm{NMR}$ and ${ }^{13} \mathrm{C}-\mathrm{NMR}$ data of $\mathbf{5 m}$ :

${ }^{1} \mathrm{H}$ NMR (400 MHz, $\mathrm{CDCl}_{3}$ ) $\delta$ 8.78-8.69 (m, 2H, CONHCO, CONH), 5.92-5.85 (m, 1H, H9), 5.82-5.67 (m, 3H, H10, H11, H23), 5.54 (dd, J = 9.9, 2.5 Hz, 1H, H22), 5.42 (dt, $\left.J=6.9,4.8 \mathrm{~Hz}, 3 \mathrm{H}, \mathrm{H} 3, \mathrm{H} 19, \mathrm{H} 1^{\prime \prime}\right), 5.00$ (d, $\left.J=11.0 \mathrm{~Hz}, 1 \mathrm{H}, \mathrm{H} 15\right), 4.78$ (d, $\left.J=3.9 \mathrm{~Hz}, 1 \mathrm{H}, \mathrm{H} 1^{\prime}\right), 4.73-4.62$ (m, 2H, H8a), 4.36 - 4.25 (m, 2H, H4", H5), 4.15-4.03 (m, 2H, H7-OH, H5"), 3.97 (d, $J=6.2$ Hz, 1H, H6), 3.93 (s, 1H, H13), 3.91-3.77 (m, 2H, H17, H5'), 3.69 (dt, $\left.J=12.2,4.4 \mathrm{~Hz}, 1 \mathrm{H}, \mathrm{H} 3{ }^{\prime \prime}\right), 3.59$ (ddd, $J=11.1$, 8.6, 4.7 Hz, 1H, H3'), 3.41 (s, 6H, H3'-OMe, H3"'-OMe), 3.33-3.17 (m, 3H, H2, H25, H4'), 2.52 (t, $J=7.4 \mathrm{~Hz}, 1 \mathrm{H}, \mathrm{H} 12), 2.42-2.17$ (m, 7H, H5-OH, H16, H24, H2"'a, $\left.\underline{\mathrm{CH}}_{2} \mathrm{CH}_{3}\right), 2.06-1.96\left(\mathrm{~m}, 2 \mathrm{H}, \mathrm{H}_{2}^{\prime} \mathrm{a}, \mathrm{H} 20 \mathrm{a}\right), 1.87\left(\mathrm{t}, J=2.0 \mathrm{~Hz}, 3 \mathrm{H}, \mathrm{H} 4 \mathrm{a}-\mathrm{CH}_{3}\right)$, 1.83-1.75 (m, 3H, H27a, H30a, H31a), 1.73-1.68 (m, 2H, H18a, H28a), 1.56 (d, $J=$ $8.2 \mathrm{~Hz}, 3 \mathrm{H}, \mathrm{H} 26, \mathrm{H} 29), 1.48$ (d, J = 7.4 Hz, 4H, H14a-CH 3 , H20b), 1.37-1.03 (m, 18H, H2' 'b, H27b, H28b, H30b, H31b, H2'b, H5'-Me, H5"'-Me, H12a-CH $\left.\mathrm{CH}_{2} \underline{\mathrm{CH}}_{3}\right), 0.92$ $\left(\mathrm{d}, J=7.1 \mathrm{~Hz}, 3 \mathrm{H}, \mathrm{H} 24 \mathrm{a}-\mathrm{CH}_{3}\right), 0.88-0.82$ (m, 1H, H18b). ${ }^{13} \mathrm{C} \mathrm{NMR}\left(100 \mathrm{MHz}, \mathrm{CDCl}_{3}\right)$ $\delta 175.7,173.6,155.2,139.4,138.0,137.8,136.1,135.0,127.7,124.7,120.5,118.2$, 118.0, 98.5, 95.6, 94.9, 81.8, 80.7, 80.3, 79.2(2-C), 73.9, 68.3, 68.3, 68.2, 67.7, 67.1, 65.4, 56.6, 56.0, 52.5, 49.5, 45.7, 41.1, 40.3, 39.7, 38.6, 36.6, 34.6, 34.3, 31.7, 31.4, 29.9, 26.9, 26.6, 26.5, 25.5, 20.2, 19.9, 18.3, 17.2, 16.6, 15.1, 8.6.

S-14: The ${ }^{1} \mathrm{H}-\mathrm{NMR}$ and ${ }^{13} \mathrm{C}-\mathrm{NMR}$ data of 5n:

${ }^{1} \mathrm{H}$ NMR (400 MHz, $\mathrm{CDCl}_{3}$ ) $\delta$ 8.78-8.69 (m, 2H, CONHCO, CONH), 5.92-5.85 
(m, 1H, H9), 5.82-5.67 (m, 3H, H10, H11, H23), 5.54 (dd, $J=9.9,2.5$ Hz, 1H, H22), $5.42\left(\mathrm{dt}, J=6.9,4.8 \mathrm{~Hz}, 3 \mathrm{H}, \mathrm{H} 3, \mathrm{H} 19, \mathrm{H} 1^{\prime \prime}\right), 5.00$ (d, $\left.J=11.0 \mathrm{~Hz}, 1 \mathrm{H}, \mathrm{H} 15\right), 4.78$ (d, $\left.J=3.9 \mathrm{~Hz}, 1 \mathrm{H}, \mathrm{H} 1^{\prime}\right), 4.73-4.62(\mathrm{~m}, 2 \mathrm{H}, \mathrm{H} 8 \mathrm{a}), 4.36-4.25\left(\mathrm{~m}, 2 \mathrm{H}, \mathrm{H} 4{ }^{\prime \prime}, \mathrm{H} 5\right)$, 4.15-4.03 (m, 2H, H7-OH, H5"), 3.97 (d, $J=6.2 \mathrm{~Hz}, 1 \mathrm{H}, \mathrm{H} 6), 3.93$ (s, 1H, H13), 3.91-3.77 (m, 2H, H17, H5'), 3.69 (dt, $J=12.2,4.4$ Hz, 1H, H3'), 3.59 (ddd, $J=11.1$, 8.6, 4.7 Hz, 1H, H3'), 3.41 (s, 6H, H3'-OMe, H3'--OMe), 3.33-3.17 (m, 3H, H2, H25, H4'), 2.52 (t, $J=7.4 \mathrm{~Hz}, 1 \mathrm{H}, \mathrm{H} 12$ ), 2.42-2.17 (m, 7H, H5-OH, H16, H24, H2"a, $\left.\mathrm{CH}_{2} \mathrm{CH}_{3}\right), 2.06-1.96\left(\mathrm{~m}, 2 \mathrm{H}, \mathrm{H} 2^{\prime} \mathrm{a}, \mathrm{H} 20 \mathrm{a}\right), 1.87\left(\mathrm{t}, J=2.0 \mathrm{~Hz}, 3 \mathrm{H}, \mathrm{H} 4 \mathrm{a}-\mathrm{CH}_{3}\right)$, 1.83-1.75 (m, 3H, H27a, H30a, H31a), 1.73-1.68 (m, 2H, H18a, H28a), 1.56 (d, $J=$ $8.2 \mathrm{~Hz}, 3 \mathrm{H}, \mathrm{H} 26, \mathrm{H} 29), 1.48$ (d, J = 7.4 Hz, 4H, H14a-CH, $\mathrm{H} 20 \mathrm{~b}), 1.37-1.03$ (m, 18H, H2 'b, H27b, H28b, H30b, H31b, H2'b, H5'-Me, H5"'-Me, H12a-CH $\mathrm{CH}_{2} \underline{\mathrm{CH}}_{3}$ ), 0.92 $\left(\mathrm{d}, J=7.1 \mathrm{~Hz}, 3 \mathrm{H}, \mathrm{H} 24 \mathrm{a}-\mathrm{CH}_{3}\right), 0.88-0.82$ (m, 1H, H18b). ${ }^{13} \mathrm{C} \mathrm{NMR}\left(100 \mathrm{MHz}, \mathrm{CDCl}_{3}\right)$ $\delta 175.7,173.6,155.2,139.4,138.0,137.8,136.1,135.0,127.7,124.7,120.5,118.2$, 118.0, 98.5, 95.6, 94.9, 81.8, 80.7, 80.3, 79.2(2-C), 73.9, 68.3, 68.3, 68.2, 67.7, 67.1, 65.4, 56.6, 56.0, 52.5, 49.5, 45.7, 41.1, 40.3, 39.7, 38.6, 36.6, 34.6, 34.3, 31.7, 31.4, 29.9, 26.9, 26.6, 26.5, 25.5, 20.2, 19.9, 18.3, 17.2, 16.6, 15.1, 8.6.

S-15: The ${ }^{1} \mathrm{H}-\mathrm{NMR}$ and ${ }^{13} \mathrm{C}-\mathrm{NMR}$ data of 5o:

${ }^{1} \mathrm{H}$ NMR (400 MHz, $\left.\mathrm{CDCl}_{3}\right) \delta 9.40(\mathrm{~s}, 1 \mathrm{H}, \mathrm{CONHCO}), 8.83(\mathrm{~d}, J=9.9 \mathrm{~Hz}, 1 \mathrm{H}$, CONH), 5.92-5.86 (m, 1H, H9), 5.83-5.66 (m, 3H, H10, H11, H23), 5.53 (dd, J = 9.9, $2.5 \mathrm{~Hz}, 1 \mathrm{H}, \mathrm{H} 22), 5.44-5.34$ (m, 3H, H3, H19, H1'), 4.99 (d, J = 10.7 Hz, 1H, H15), $4.77\left(\mathrm{~d}, J=3.8 \mathrm{~Hz}, 1 \mathrm{H}, \mathrm{H} 1^{\prime}\right), 4.73-4.61(\mathrm{~m}, 2 \mathrm{H}, \mathrm{H} 8 \mathrm{a}), 4.31(\mathrm{dd}, J=10.0,4.2 \mathrm{~Hz}, 2 \mathrm{H}$, H4", H5), 4.11-4.02 (m, 1H, H5"), 3.97 (d, J = 6.2 Hz, 1H, H6), 3.93 (s, 1H, H13), 3.90-3.78 (m, 2H, H17, H5'), 3.68 (dt, $\left.J=12.3,4.3 \mathrm{~Hz}, 1 \mathrm{H}, \mathrm{H} 3{ }^{\prime \prime}\right), 3.64-3.55$ (m, 2H, H3', $\underline{\mathrm{C}}_{3} \underline{\mathrm{H}}_{5}$ ), 3.40 (d, J = $1.8 \mathrm{~Hz}, 6 \mathrm{H}, \mathrm{H}^{\prime}{ }^{\prime}-\mathrm{OMe}, \mathrm{H} 3^{\prime \prime}-\mathrm{OMe}$ ), 3.34-3.17 (m, 3H, H2, H25, H4'), 2.59-2.47 (m, 2H, H12, H5-OH), 2.42 (t, $J=7.1 \mathrm{~Hz}, 1 \mathrm{H}, \underline{\mathrm{C}}_{3} \underline{\mathrm{H}}_{5}$ ), 2.34-2.18 (m, 4H, H16, H24, H2'”a), 2.15-2.08 (m, 1H, $\underline{\mathrm{C}}_{3} \underline{\mathrm{H}}_{5}$ ), 2.06-1.91 (m, 2H, H2'a, H20a), 1.86 (d, $J=2.0 \mathrm{~Hz}, 3 \mathrm{H}, \mathrm{H} 4 \mathrm{a}-\mathrm{CH}_{3}$ ), 1.83-1.73 (m, 3H, H27a, H30a, H31a), 1.72-1.64 (m, 2H, H18a, H28a), 1.55 (d, $J=3.6$ Hz, 3H, H26, H29), 1.46 (d, $J=18.7$ Hz, 4H, H14a- $\left.\mathrm{CH}_{3}, \mathrm{H} 20 \mathrm{~b}\right), 1.34-1.03$ (m, 15H, H2'b, H27b, H28b, H30b, H31b, H2'b, H5'-Me, H5"'-Me, H12a-CH 3 ), 0.92 (d, $\left.J=7.2 \mathrm{~Hz}, 3 \mathrm{H}, \mathrm{H} 24 \mathrm{a}-\mathrm{CH}_{3}\right), 0.89-0.78$ (m, 3H, 
$\left.\mathrm{H} 18 \mathrm{~b}, \underline{\mathrm{C}}_{2} \underline{\mathrm{H}}_{5}\right) .{ }^{13} \mathrm{C} \mathrm{NMR}\left(100 \mathrm{MHz}, \mathrm{CDCl}_{3}\right) \delta 175.8,173.6,155.2,139.4,138.0,137.8$, 136.1, 135.0, 127.7, 124.7, 120.5, 118.2, 118.1, 98.5, 95.7, 94.9, 81.8, 80.7, 80.3, 79.2, 73.8, 68.3, 68.3, 68.1, 67.7, 67.1, 65.4, 56.6, 55.9, 52.5, 49.5, 45.7, 44.1, 41.1, 40.3, 39.7, 38.6, 36.6, 34.5, 34.3, 31.7, 31.4, 30.0, 27.0, 26.9, 26.6, 26.5, 25.5, 20.2, 19.9, 18.3, 17.2, 16.6, 15.2, 15.1 .

S-16: The ${ }^{1} \mathrm{H}-\mathrm{NMR}$ and ${ }^{13} \mathrm{C}-\mathrm{NMR}$ data of 5p:

${ }^{1} \mathrm{H}$ NMR (400 MHz, $\left.\mathrm{CDCl}_{3}\right) \delta 8.79(\mathrm{~d}, J=9.9 \mathrm{~Hz}, 1 \mathrm{H}, \mathrm{CONHCO}), 8.60(\mathrm{~s}, 1 \mathrm{H}$, CONH), 5.93-5.85 (m, 1H, H9), 5.83-5.66 (m, 3H, H10, H11, H23), 5.54 (dd, $J=9.9$, 2.5 Hz, 1H, H22), 5.48-5.36 (m, 3H, H3, H19, H1"), 5.04-4.97 (m, 1H, H15), 4.78 (d, $\left.J=4.0 \mathrm{~Hz}, 1 \mathrm{H}, \mathrm{H1} 1^{\prime}\right), 4.73-4.64$ (m, 2H, H8a), 4.35-4.26 (m, 2H, H4", H5), 4.14-4.03 (m, 2H, H7-OH, H5"), 3.97 (d, $J=6.2 \mathrm{~Hz}, 1 \mathrm{H}, \mathrm{H6}$ ), 3.93 (s, 1H, H13), 3.90-3.78 (m, 2H, H17, H5'), 3.69 (dt, $J=12.2,4.4$ Hz, 1H, H3"), 3.64-3.55 (m, 1H, H3'), 3.41 (d, $J$ $=3.6 \mathrm{~Hz}, 6 \mathrm{H}, \mathrm{H} 3{ }^{\prime}-\mathrm{OMe}, \mathrm{H} 3{ }^{\prime \prime}-\mathrm{OMe}$ ), 3.34-3.16 (m, 3H, H2, H25, H4), 2.52 (t, $J=7.2$ $\mathrm{Hz}, 1 \mathrm{H}, \mathrm{H} 12), 2.42-2.15$ (m, 6H, H5-OH, H16, H24, H2"a, $\left.\underline{\mathrm{C}}_{6} \underline{\mathrm{H}}_{11}\right), 2.06-1.97$ (m, 2H, H2'a, H20a), 1.94-1.84 (m, 5H, H4a-CH $\underline{\mathrm{C}}_{6} \underline{\mathrm{H}_{11}}$ ), 1.84-1.74 (m, 5H, H27a, H30a, H31a, $\left.\underline{\mathrm{C}}_{6} \underline{\mathrm{H}}_{11}\right), 1.73-1.65$ (m, 2H, H18a, H28a), 1.55 (d, $\left.J=7.6 \mathrm{~Hz}, 3 \mathrm{H}, \mathrm{H} 26, \mathrm{H} 29\right)$, 1.51-1.40 (m, 6H, H14a-CH $\mathrm{CH}_{3}, \mathrm{H} 20 \mathrm{~b}, \underline{\mathrm{C}}_{6} \underline{H}_{11}$ ), 1.35-1.10 (m, 19H, H2"b, H27b, H28b, H30b, H31b, H2'b, H5'-Me, H5"'-Me, H12a- $\left.\mathrm{CH}_{3}, \underline{\mathrm{C}}_{6} \underline{H}_{11}\right), 0.92$ (d, $J=7.2 \mathrm{~Hz}, 3 \mathrm{H}$, $\left.\mathrm{H} 24 \mathrm{a}-\mathrm{CH}_{3}\right), 0.88-0.81$ (m, 1H, H18b). ${ }^{13} \mathrm{C}$ NMR $\left(100 \mathrm{MHz}, \mathrm{CDCl}_{3}\right) \delta 178.0,173.6$, 155.3, 139.4, 138.1, 137.9, 136.2, 135.0, 127.7, 124.7, 120.5, 118.2, 118.0, 98.6, 95.7, $94.9,81.8,80.8,80.3,79.2,73.9,68.3,68.3,68.1,67.7,67.1,65.4,56.6,56.0,52.5$, 49.5, 45.7, 45.6, 41.1, 40.3, 39.7, 38.6, 36.6, 34.5, 34.3, 31.7, 31.4, 30.0, 29.0, 28.9, 26.9, 26.6, 26.5, 25.5, 25.5, 25.5, 25.5, 20.2, 19.9, 18.3, 17.3, 16.6, 15.1 .

S-17: The ${ }^{1} \mathrm{H}-\mathrm{NMR}$ and ${ }^{13} \mathrm{C}-\mathrm{NMR}$ data of 5q:

${ }^{1} \mathrm{H}$ NMR $\left(400 \mathrm{MHz}, \mathrm{CDCl}_{3}\right) \delta 8.76(\mathrm{t}, J=5.0 \mathrm{~Hz}, 2 \mathrm{H}, \mathrm{CONHCO}, \mathrm{CONH})$, 5.93-5.85 (m, 1H, H9), 5.82-5.67 (m, 3H, H10, H11, H23), 5.54 (dd, $J=9.9,2.5 \mathrm{~Hz}$, 1H, H22), 5.47-5.35 (m, 3H, H3, H19, H1"), 5.00 (d, $J=10.6 \mathrm{~Hz}, 1 \mathrm{H}, \mathrm{H} 15$ ), 4.80-4.76 (m, 1H, H1'), 4.73-4.62 (m, 2H, H8a), 4.31 (d, J=12.5 Hz, 2H, H4", H5), 4.13-4.04 (m, 2H, H7-OH, H5"), 3.97 (d, $J=6.2$ Hz, 1H, H6), 3.93 (s, 1H, H13), 3.84 (ddd, $J=15.6,9.5,5.4 \mathrm{~Hz}, 2 \mathrm{H}, \mathrm{H17}, \mathrm{H} 5^{\prime}$ ), 3.69 (dt, $\left.J=12.3,4.3 \mathrm{~Hz}, 1 \mathrm{H}, \mathrm{H} 3^{\prime \prime}\right), 3.59$ 
(ddd, $J=11.2,8.6,4.7 \mathrm{~Hz}, 1 \mathrm{H}, \mathrm{H} 3$ '), 3.41 (s, 6H, H3'-OMe, H3"'-OMe), 3.33-3.18 (m, 3H, H2, H25, H4), 2.52 (t, $J=7.6 \mathrm{~Hz}, 1 \mathrm{H}, \mathrm{H} 12), 2.37$ (d, $J=8.2 \mathrm{~Hz}, 1 \mathrm{H}, \mathrm{H} 5-\mathrm{OH})$, 2.35-2.18 (m, 6H, H16, H24, H2" a, $\underline{\mathrm{CH}}_{2} \mathrm{CH}_{2} \mathrm{CH}_{2} \mathrm{CH}_{2} \mathrm{CH}_{3}$ ), 2.01 (dt, $J=11.3,5.2 \mathrm{~Hz}$, 2H, H2' a, H20a), 1.87 (d, $\left.J=2.1 \mathrm{~Hz}, 3 \mathrm{H}, \mathrm{H} 4 \mathrm{a}-\mathrm{CH}_{3}\right), 1.83-1.75$ (m, 3H, H27a, H30a, H31a), 1.72-1.64 (m, 4H, H18a, H28a, $\mathrm{CH}_{2} \mathrm{CH}_{2} \mathrm{CH}_{2} \mathrm{CH}_{2} \mathrm{CH}_{3}$ ), 1.56 (d, $J=8.6 \mathrm{~Hz}, 3 \mathrm{H}$, H26, H29), 1.48 (d, $\left.J=7.0 \mathrm{~Hz}, 4 \mathrm{H}, \mathrm{H} 14 \mathrm{a}-\mathrm{CH}_{3}, \mathrm{H} 20 \mathrm{~b}\right), 1.35-1.11$ (m, 19H, H2'b, H27b, H28b, H30b, H31b, H2'b, H5'-Me, H5"'-Me, H12a- $\mathrm{CH}_{3}, \mathrm{CH}_{2} \mathrm{CH}_{2} \mathrm{CH}_{2} \mathrm{CH}_{2} \mathrm{CH}_{3}$ ), 0.96-0.80 (m, 7H, H24a-CH $\left.3, \mathrm{H} 18 \mathrm{~b}, \mathrm{CH}_{2} \mathrm{CH}_{2} \mathrm{CH}_{2} \mathrm{CH}_{2} \mathrm{CH}_{3}\right) .{ }^{13} \mathrm{C}$ NMR (100 MHz, $\left.\mathrm{CDCl}_{3}\right) \delta 175.2,173.6,155.2,139.4,138.0,137.8,136.1,135.0,127.7,124.6,120.5$, 118.2, 118.0, 98.5, 95.7, 94.9, 81.8, 80.7, 80.3, 79.2, 73.9, 68.3, 68.2, 67.7, 67.1, 65.4, 56.6, 56.0, 52.5, 49.5, 45.7, 41.1, 40.3, 39.7, 38.6, 36.8, 36.6, 35.9, 34.6, 34.3, 31.7, $31.4,31.2,30.0,26.9,26.6,26.5,25.5,24.3,22.3,20.2$, 19.9, 18.3, 17.2, 16.6, 15.1, 13.8.

S-18: The ${ }^{1} \mathrm{H}-\mathrm{NMR}$ and ${ }^{13} \mathrm{C}-\mathrm{NMR}$ data of 5r:

${ }^{1} \mathrm{H}$ NMR (400 MHz, $\left.\mathrm{CDCl}_{3}\right) \delta 8.80(\mathrm{~d}, J=9.9 \mathrm{~Hz}, 1 \mathrm{H}, \mathrm{CONHCO}), 8.49$ (d, $J=$ $14.1 \mathrm{~Hz}, 1 \mathrm{H}, \mathrm{CONH}), 8.13(\mathrm{td}, J=8.9,6.4 \mathrm{~Hz}, 1 \mathrm{H}, \mathrm{Ph}), 7.05$ (ddd, $J=9.3,7.4,2.5$ $\mathrm{Hz}, 1 \mathrm{H}, \mathrm{Ph}), 6.94$ (ddd, $J=12.1,8.3,2.4 \mathrm{~Hz}, 1 \mathrm{H}, \mathrm{Ph}), 5.92-5.86$ (m, 1H, H9), 5.84-5.67 (m, 3H, H10, H11, H23), 5.54 (dd, $J=9.9,2.5$ Hz, 1H, H22), 5.50-5.35 (m, 3H, H3, H19, H1'), 5.00 (d, $J=10.7 \mathrm{~Hz}, 1 \mathrm{H}, \mathrm{H} 15), 4.79$ (d, $\left.J=3.8 \mathrm{~Hz}, 1 \mathrm{H}, \mathrm{H} 1{ }^{\prime}\right)$, 4.74-4.62 (m, 2H, H8a), 4.39 (dd, $\left.J=10.1,3.5 \mathrm{~Hz}, 1 \mathrm{H}, \mathrm{H} 4^{\prime \prime}\right), 4.30$ (d, $J=6.3 \mathrm{~Hz}, 1 \mathrm{H}$, H5), 4.12 (dt, $\left.J=7.5,5.9 \mathrm{~Hz}, 1 \mathrm{H}, \mathrm{H} 5^{\prime \prime}\right), 4.06$ (s, 1H, H7-OH), 3.98 (d, J=6.2 Hz, 1H, H6), 3.94 (s, 1H, H13), 3.92-3.80 (m, 2H, H17, H5), 3.74 (dt, J = 12.2, 4.4 Hz, 1H, H3"), 3.62 (ddd, $J=11.0,8.7,4.7$ Hz, 1H, H3'), 3.45 (s, 3H, H3'-OMe), 3.43 (s, 3H, H3"'-OMe), 3.34-3.22 (m, 3H, H2, H25, H4'), 2.53 (t, J = 7.6 Hz, 1H, H12), 2.40-2.18 (m, 5H, H5-OH, H16, H24, H2"a), 2.10-1.96 (m, 2H, H2'a, H20a), 1.87 (s, 3H, H4a-CH $_{3}$, 1.83-1.76 (m, 3H, H27a, H30a, H31a), 1.74-1.67 (m, 2H, H18a, H28a), 1.56 (d, $J=8.7 \mathrm{~Hz}, 3 \mathrm{H}, \mathrm{H} 26, \mathrm{H} 29), 1.49$ (d, $J=6.7 \mathrm{~Hz}, 4 \mathrm{H}, \mathrm{H} 14 \mathrm{a}-\mathrm{CH}_{3}, \mathrm{H} 20 \mathrm{~b}$ ), 1.36-1.12 (m, 15H, H2'b, H27b, H28b, H30b, H31b, H2 b, H5'-Me, H5"-Me, $\left.\mathrm{H} 12 \mathrm{a}-\mathrm{CH}_{3}\right), 0.93$ (d, $\left.J=7.1 \mathrm{~Hz}, 3 \mathrm{H}, \mathrm{H} 24 \mathrm{a}-\mathrm{CH}_{3}\right), 0.88-0.82$ (m, 1H, H18b). ${ }^{13} \mathrm{C}$ NMR $\left(100 \mathrm{MHz}, \mathrm{CDCl}_{3}\right) \delta 173.5,163.1,153.6,139.5,138.0,137.8,136.1,135.0,134.0$, 
133.9, 127.7, 124.7, 120.4, 118.2, 118.0, 116.3, 113.0, 112.8, 98.7, 95.7, 94.8, 81.8, 81.0, 80.3, 79.2, 79.2, 77.3, 73.8, 68.4, 68.3, 68.2, 67.7, 67.1, 65.4, 56.6, 56.1, 52.5, 49.9, 45.7, 40.3, 39.7, 38.6, 36.6, 34.6, 34.3, 31.8, 31.4, 30.0, 26.9, 26.6, 26.4, 25.5, 20.2, 19.9, 18.3, 17.2, 16.6, 15.1 .

S-19: The ${ }^{1} \mathrm{H}-\mathrm{NMR}$ and ${ }^{13} \mathrm{C}-\mathrm{NMR}$ data of 5s:

${ }^{1} \mathrm{H}$ NMR (400 MHz, $\left.\mathrm{CDCl}_{3}\right) \delta 8.76(\mathrm{~d}, J=9.8 \mathrm{~Hz}, 1 \mathrm{H}, \mathrm{CONHCO}), 8.59(\mathrm{~d}, J=$ $14.7 \mathrm{~Hz}, 1 \mathrm{H}, \mathrm{CONH}), 7.79$ (ddd, $J=8.8,5.8,3.2 \mathrm{~Hz}, 1 \mathrm{H}, \mathrm{Ph}), 7.31-7.23(\mathrm{~m}, 1 \mathrm{H})$, 7.18 (ddd, $J=10.9,9.1,4.1 \mathrm{~Hz}, 1 \mathrm{H}, \mathrm{Ph}), 5.94-5.86$ (m, 1H, H9), 5.83-5.67 (m, 3H, H10, H11, H23), 5.54 (dd, $J=9.9,2.6 \mathrm{~Hz}, 1 \mathrm{H}, \mathrm{H} 22), 5.50-5.37$ (m, 3H, H3, H19, H1' ${ }^{\prime \prime}, 5.01$ (d, $\left.J=10.7 \mathrm{~Hz}, 1 \mathrm{H}, \mathrm{H} 15\right), 4.79$ (d, $\left.J=3.7 \mathrm{~Hz}, 1 \mathrm{H}, \mathrm{H} 1^{\prime}\right), 4.75-4.62$ (m, 2H, H8a), 4.39 (dd, $\left.J=10.0,3.5 \mathrm{~Hz}, 1 \mathrm{H}, \mathrm{H} 4{ }^{\prime \prime}\right), 4.33-4.26$ (m, 1H, H5), 4.17 - 4.08 (m, 1H, H5"), 4.05 (s, 1H, H7-OH), 3.98 (d, J = 6.2 Hz, 1H, H6), 3.94 (s, 1H, H13), 3.91-3.81 (m, 2H, H17, H5'), 3.74 (dt, $J=12.2,4.4$ Hz, 1H, H3'), 3.62 (ddd, $J=11.2$, 8.5, 4.7 Hz, 1H, H3'), 3.45 (s, 3H, H3'-OMe), 3.44 (s, 3H, H3"'-OMe), 3.35-3.21 (m, 3H, H2, H25, H4), 2.53 (t, $J=7.8 \mathrm{~Hz}, 1 \mathrm{H}, \mathrm{H} 12$ ), 2.37-2.18 (m, 5H, H5-OH, H16, H24, H2"a), 2.12-1.96 (m, 2H, H2'a, H20a), 1.88 (m, 3H, H4a-CH $)$, 1.83-1.75 (m, 3H, H27a, H30a, H31a), 1.73-1.66 (m, 2H, H18a, H28a), 1.55 (d, J=3.5 Hz, 3H, H26, H29), 1.49 (d, $J=7.1 \mathrm{~Hz}, 4 \mathrm{H}, \mathrm{H} 14 \mathrm{a}-\mathrm{CH}_{3}, \mathrm{H} 20 \mathrm{~b}$ ), 1.38-1.08 (m, 15H, H2'b, H27b, H28b, H30b, H31b, H2 b, H5'-Me, H5"-Me, H12a-CH $), 0.93$ (d, J = $7.2 \mathrm{~Hz}, 3 \mathrm{H}$, $\left.\mathrm{H} 24 \mathrm{a}-\mathrm{CH}_{3}\right), 0.88-0.81$ (m, 1H, H18b). ${ }^{13} \mathrm{C} \mathrm{NMR}\left(100 \mathrm{MHz}, \mathrm{CDCl}_{3}\right) \delta 173.5,162.8$, 160.0 , 157.6, 157.6, 155.2, 153.4, 139.5, 138.0, 137.8, 136.1, 135.0, 127.7, 124.7, 120.4, 118.2, 118.0, 117.9, 98.7, 95.7, 94.8, 81.8, 81.0, 80.3, 79.2, 79.2, 77.3, 73.8, 68.3, 68.2, 68.1, 67.7, 67.0, 65.3, 56.6, 56.1, 52.5, 49.9, 45.7, 40.3, 39.7, 38.6, 36.6, 34.6, 34.3, 31.7, 31.4, 30.0, 26.9, 26.6, 26.5, 25.5, 20.2, 19.9, 18.3, 17.2, 16.6, 15.1.

S-20: The ${ }^{1} \mathrm{H}-\mathrm{NMR}$ and ${ }^{13} \mathrm{C}-\mathrm{NMR}$ data of $\mathbf{5 t}$ :

${ }^{1} \mathrm{H}$ NMR (400 MHz, $\left.\mathrm{CDCl}_{3}\right) \delta 8.58(\mathrm{~d}, J=9.8 \mathrm{~Hz}, 1 \mathrm{H}, \mathrm{CONHCO}), 8.24(\mathrm{~s}, 1 \mathrm{H}$, CONH), 7.47 (ddd, $J=14.6,8.4,6.2 \mathrm{~Hz}, 1 \mathrm{H}, \mathrm{Ph}), 7.01$ (t, $J=8.5 \mathrm{~Hz}, 2 \mathrm{H}, \mathrm{Ph}), 5.89$ (d, $J=10.3 \mathrm{~Hz}, 1 \mathrm{H}, \mathrm{H} 9), 5.83-5.67$ (m, 3H, H10, H11, H23), 5.54 (dd, $J=9.9,2.5 \mathrm{~Hz}$, 1H, H22), 5.49-5.36 (m, 3H, H3, H19, H1 "), 5.01 (d, J= 10.7 Hz, 1H, H15), 4.79 (d, $\left.J=3.8 \mathrm{~Hz}, 1 \mathrm{H}, \mathrm{H} 1{ }^{\prime}\right), 4.73-4.62$ (m, 2H, H8a), 4.36 (d, $\left.J=10.2 \mathrm{~Hz}, 1 \mathrm{H}, \mathrm{H} 4^{\prime \prime}\right), 4.30$ (s, 
1H, H5), 4.18-4.05 (m, 2H, H5", H7-OH), 3.97 (d, J=6.2 Hz, 1H, H6), 3.94 (s, 1H, H13), 3.91-3.80 (m, 2H, H17, H5'), 3.73 (dt, $J=12.3,4.4$ Hz, 1H, H3"), 3.65-3.56 (m, 1H, H3'), 3.43 (d, J = 1.4 Hz, 6H, H3'-OMe, H3"'-OMe), 3.35-3.22 (m, 3H, H2, H25, H4'), 2.58-2.48 (m, 1H, H12), 2.39-2.19 (m, 5H, H5-OH, H16, H24, H2"a), 2.06-1.94 (m, 2H, H2' a, H20a), 1.88 (d, $J=2.2 \mathrm{~Hz}, 3 \mathrm{H}, \mathrm{H} 4 \mathrm{a}-\mathrm{CH}_{3}$ ), 1.83-1.76 (m, 3H, H27a, H30a, H31a), 1.72-1.65 (m, 2H, H18a, H28a), 1.55 (d, J = 9.1 Hz, 3H, H26, H29), 1.48 (d, $J=10.3 \mathrm{~Hz}, 4 \mathrm{H}, \mathrm{H} 14 \mathrm{a}-\mathrm{CH}_{3}, \mathrm{H} 20 \mathrm{~b}$ ), 1.34-1.12 (m, 15H, H2'”b, H27b, H28b, $\mathrm{H} 30 \mathrm{~b}, \mathrm{H} 31 \mathrm{~b}, \mathrm{H} 2$ 'b, H5'-Me, H5"'-Me, H12a-CH3), $0.93(\mathrm{~d}, J=7.2 \mathrm{~Hz}, 3 \mathrm{H}$, $\left.\mathrm{H} 24 \mathrm{a}-\mathrm{CH}_{3}\right), 0.89-0.82$ (m, 1H, H18b). ${ }^{13} \mathrm{C} \mathrm{NMR}\left(100 \mathrm{MHz}, \mathrm{CDCl}_{3}\right) \delta 173.6,161.5$, $161.3,158.7,153.8,139.4,138.0,137.8,136.1,135.0,133.0,127.7,124.7,120.5$, 118.2, 118.0, 112.3, 112.1, 98.6, 95.7, 94.9, 81.8, 80.9, 80.2, 79.3, 79.2, 73.8, 68.4, 68.2, 67.7, 67.1, 65.3, 56.6, 56.0, 52.5, 49.9, 45.8, 41.1, 40.3, 39.7, 38.6, 36.6, 34.5, 34.3, 31.7, 31.4, 30.0, 29.6, 27.0, 26.6, 26.4, 25.5, 20.2, 19.8, 18.3, 17.3, 16.6, 15.1.

S-21: The ${ }^{1} \mathrm{H}-\mathrm{NMR}$ and ${ }^{13} \mathrm{C}-\mathrm{NMR}$ data of 5u:

${ }^{1} \mathrm{H}$ NMR (400 MHz, $\left.\mathrm{CDCl}_{3}\right) \delta 8.91(\mathrm{~d}, J=9.8 \mathrm{~Hz}, 1 \mathrm{H}, \mathrm{CONHCO}), 8.44(\mathrm{~s}, 1 \mathrm{H}$, CONH), 6.98 (d, $J=2.3 \mathrm{~Hz}, 2 \mathrm{H}, \mathrm{Ph}), 6.64(\mathrm{t}, J=2.3 \mathrm{~Hz}, 1 \mathrm{H}, \mathrm{Ph}), 5.93-5.86$ (m, 1H, H9), 5.83-5.67 (m, 3H, H10, H11, H23), 5.54 (dd, J = 9.9, $2.6 \mathrm{~Hz}, 1 \mathrm{H}, \mathrm{H} 22)$, 5.50-5.37 (m, 3H, H3, H19, H1 "), 5.01 (d, $J=10.6$ Hz, 1H, H15), 4.81-4.76 (m, 1H, H1'), 4.73-4.63 (m, 2H, H8a), 4.43-4.35 (m, 1H, H4"), 4.30 (s, 1H, H5), 4.16-4.04 (m, 2H, H5", H7-OH), 3.98 (d, J=6.2 Hz, 1H, H6), 3.94 (s, 1H, H13), 3.91-3.78 (m, 2H, H17, H5'), $3.83\left(\mathrm{~s}, 6 \mathrm{H},\left(\mathrm{OCH}_{3}\right)_{2}\right), 3.73\left(\mathrm{dt}, J=12.2,4.4 \mathrm{~Hz}, 1 \mathrm{H}, \mathrm{H} 3{ }^{\prime \prime}\right), 3.61$ (ddd, $J=$ 11.2, 8.6, 4.8 Hz, 1H, H3'), 3.43 (d, $J=2.4$ Hz, 6H, H3'-OMe, H3"'-OMe), 3.34-3.21 (m, 3H, H2, H25, H4'), 2.53 (t, $J=7.7$ Hz, 1H, H12), 2.41-2.19 (m, 5H, H5-OH, H16, H24, H2"a), 2.11-1.96 (m, 2H, H2'a, H20a), 1.87 (s, 3H, H4a-CH ${ }_{3}$ ) 1.83-1.74 (m, 3H, H27a, H30a, H31a), 1.72-1.64 (m, 2H, H18a, H28a), 1.56 (d, $J=10.0$ Hz, 3H, H26, H29), 1.49 (d, $J=7.4$ Hz, 4H, H14a-CH, $\mathrm{H} 20 \mathrm{~b}$ ), 1.35-1.12 (m, 15H, H2'b, H27b, H28b, H30b, H31b, H2'b, H5'-Me, H5"-Me, H12a-CH $), 0.92$ (d, J = $7.1 \mathrm{~Hz}, 3 \mathrm{H}$, $\left.\mathrm{H} 24 \mathrm{a}-\mathrm{CH}_{3}\right), 0.86(\mathrm{~d}, J=12.2 \mathrm{~Hz}, 1 \mathrm{H}, \mathrm{H} 18 \mathrm{~b}) .{ }^{13} \mathrm{C} \mathrm{NMR}\left(100 \mathrm{MHz}, \mathrm{CDCl}_{3}\right) \delta 173.6$, 167.9, 160.9(2-C), 154.6, 139.5, 138.0, 137.8, 136.2, 135.0, 134.5, 127.7, 124.7, 120.5, 118.2, 118.1, 105.4(2-C), 105.3, 98.7, 95.7, 94.9, 81.8, 81.0, 80.4, 79.2, 73.9, 
68.4, 68.3, 68.2, 67.7, 67.1, 65.5, 56.6, 56.0, 55.6(2-C), 52.5, 49.8, 45.7, 41.1, 40.3, 39.7, 38.6, 36.6, 34.6, 34.3, 31.8, 31.4, 30.0, 26.9, 26.6, 26.5, 25.5, 20.2, 19.9, 18.3, $17.2,16.6,15.1$.

S-22: The ${ }^{1} \mathrm{H}-\mathrm{NMR}$ and ${ }^{13} \mathrm{C}-\mathrm{NMR}$ data of $\mathbf{5 v}$ :

${ }^{1} \mathrm{H}$ NMR (400 MHz, $\left.\mathrm{CDCl}_{3}\right) \delta 8.93(\mathrm{~d}, J=9.8 \mathrm{~Hz}, 1 \mathrm{H}, \mathrm{CONHCO}), 8.28(\mathrm{~s}, 1 \mathrm{H}$, CONH), 7.07 (d, $J=1.5 \mathrm{~Hz}, 2 \mathrm{H}, \mathrm{Ph}), 5.90$ (d, $J=9.7 \mathrm{~Hz}, 1 \mathrm{H}, \mathrm{H} 9), 5.83-5.67$ (m, 3H, H10, H11, H23), 5.54 (dd, $J=9.9,2.5$ Hz, 1H, H22), 5.49-5.33 (m, 3H, H3, H19, H1" ), 5.01 (d, $J=10.7 \mathrm{~Hz}, 1 \mathrm{H}, \mathrm{H} 15), 4.79$ (d, $\left.J=3.8 \mathrm{~Hz}, 1 \mathrm{H}, \mathrm{H} 1^{\prime}\right), 4.68$ (d, $J=3.1$ Hz, 2H, H8a), 4.37 (d, $\left.J=9.7 \mathrm{~Hz}, 1 \mathrm{H}, \mathrm{H} 4{ }^{\prime \prime}\right), 4.30$ (t, $\left.J=7.3 \mathrm{~Hz}, 1 \mathrm{H}, \mathrm{H} 5\right), 4.15-4.09$ (m, 1H, H5"), 4.07 (s, 1H, H7-OH), 3.98 (d, J = 6.3 Hz, 1H, H6), 3.97-3.79 (m, 3H, H13, H17, H5'), 3.91 (d, $\left.J=1.6 \mathrm{~Hz}, 9 \mathrm{H},\left(\mathrm{OCH}_{3}\right)_{3}\right), 3.77-3.70$ (m, 1H, H3'), 3.65-3.57 (m, 1H, H3'), 3.43 (d, $J=2.0$ Hz, 6H, H3'-OMe, H3"'-OMe), 3.34-3.21 (m, 3H, H2, H25, H4'), 2.53 (t, $J=7.7$ Hz, 1H, H12), 2.36 (d, $J=8.2 \mathrm{~Hz}, 1 \mathrm{H}, \mathrm{H} 5-\mathrm{OH}), 2.33-2.19$ (m, 4H, H16, H24, H2"a), 2.11-1.98 (m, 2H, H2'a, H20a), 1.88 (s, 3H, H4a-CH ${ }_{3}$ ), 1.83-1.74 (m, 3H, H27a, H30a, H31a), 1.73-1.63 (m, 2H, H18a, H28a), 1.57 (d, $J=$ $13.9 \mathrm{~Hz}, 3 \mathrm{H}, \mathrm{H} 26, \mathrm{H} 29), 1.49$ (d, $\left.J=7.1 \mathrm{~Hz}, 4 \mathrm{H}, \mathrm{H} 14 \mathrm{a}-\mathrm{CH}_{3}, \mathrm{H} 20 \mathrm{~b}\right), 1.34-1.14$ (m, 15H, H2'b, H27b, H28b, H30b, H31b, H2'b, H5'-Me, H5"-Me, H12a-CH 3 ), 0.93 (d, $\left.J=7.2 \mathrm{~Hz}, 3 \mathrm{H}, \mathrm{H} 24 \mathrm{a}-\mathrm{CH}_{3}\right), 0.86(\mathrm{~d}, J=12.9 \mathrm{~Hz}, 1 \mathrm{H}, \mathrm{H} 18 \mathrm{~b}) .{ }^{13} \mathrm{C} \mathrm{NMR}(100 \mathrm{MHz}$, $\left.\mathrm{CDCl}_{3}\right) \delta 173.6,167.8,154.8,153.1(2-\mathrm{C}), 142.1,139.61,138.0,137.8,136.2,135.1$, $127.7,127.5,124.7,120.5,118.2,118.1,105.2,98.8,95.6,94.9,81.8,81.2,80.4,79.2$, $79.2,73.8,68.4,68.3,68.2,67.7,67.1,65.4,60.9,56.6,56.4(2-\mathrm{C}), 55.9,52.5,49.9$, $45.8,41.1,40.3,39.7,38.6,36.6,34.6,34.3,31.7,31.4,30.0,26.9,26.6,26.5,25.5$, 20.2, 19.9, 18.3, 17.2, 16.6, 15.1 .

S-23: The ${ }^{1} \mathrm{H}-\mathrm{NMR}$ and ${ }^{13} \mathrm{C}-\mathrm{NMR}$ data of $\mathbf{6 b}$ :

${ }^{1} \mathrm{H}$ NMR (400 MHz, CDC 3 ) $\delta 11.04(\mathrm{~d}, J=9.9 \mathrm{~Hz}, 1 \mathrm{H}, \mathrm{CONHCS}), 9.60$ (d, $J=$ $14.6 \mathrm{~Hz}, 1 \mathrm{H}, \mathrm{CSNH}), 8.07$ (td, $J=7.9,1.9 \mathrm{~Hz}, 1 \mathrm{H}, \mathrm{Ph}), 7.60$ (dt, $J=8.0,2.4 \mathrm{~Hz}, 1 \mathrm{H}$, $\mathrm{Ph}), 7.32$ (t, $J=7.6 \mathrm{~Hz}, 1 \mathrm{H}, \mathrm{Ph}), 7.20$ (dd, $J=12.2,8.3 \mathrm{~Hz}, 1 \mathrm{H}, \mathrm{Ph}), 5.93-5.85(\mathrm{~m}, 1 \mathrm{H}$, H9), 5.83-5.67 (m, 3H, H10, H11, H23), 5.58-5.49 (m, 2H, H22, H1"), 5.47-5.31 (m, 2H, H3, H19), 5.10 (dd, $\left.J=9.9,3.6 \mathrm{~Hz}, 1 \mathrm{H}, \mathrm{H} 4^{\prime \prime}\right), 5.00$ (d, $\left.J=10.6 \mathrm{~Hz}, 1 \mathrm{H}, \mathrm{H} 15\right)$, $\left.4.79(\mathrm{~d}, J=3.8 \mathrm{~Hz}, 1 \mathrm{H}, \mathrm{H1})^{\prime}\right), 4.74-4.62(\mathrm{~m}, 2 \mathrm{H}, \mathrm{H} 8 \mathrm{a}), 4.29$ (d, $\left.J=6.1 \mathrm{~Hz}, 1 \mathrm{H}, \mathrm{H} 5\right)$, 
4.18 (q, $\left.J=6.5 \mathrm{~Hz}, 1 \mathrm{H}, \mathrm{H} 5^{\prime \prime}\right), 4.06$ (s, 1H, H7-OH), 3.97 (d, $\left.J=6.2 \mathrm{~Hz}, 1 \mathrm{H}, \mathrm{H} 6\right)$, 3.94 (s, 1H, H13), 3.91-3.77 (m, 3H, H17, H5', H3'), 3.62 (ddd, J = 11.3, 8.4, 4.7 Hz, 1H, H3'), 3.51 (s, 3H, H3"-OMe), 3.43 (s, 3H, H3'-OMe), 3.34-3.21 (m, 3H, H2, H25, H4'), 2.57-2.47 (m, 1H, H12), 2.39 (s, 1H, H5-OH), 2.35-2.18 (m, 4H, H16, H24, H2'a), 2.14 (dd, $J=13.7,4.9$ Hz, 1H, H2'a), 2.04-1.96 (m, 1H, H20a), 1.87 (t, $J=2.0$ $\mathrm{Hz}, 3 \mathrm{H}, \mathrm{H} 4 \mathrm{a}-\mathrm{CH}_{3}$ ), 1.82-1.75 (m, 3H, H27a, H30a, H31a), 1.73-1.65 (m, 2H, H18a, H28a), 1.56 (d, $J=8.4$ Hz, 3H, H26, H29), 1.48 (d, J=7.7 Hz, 4H, H14a-CH, H20b), 1.36-1.10 (m, 15H, H2'b, H27b, H28b, H30b, H31b, H2'b, H5'-Me, H5"'-Me, $\left.\mathrm{H} 12 \mathrm{a}-\mathrm{CH}_{3}\right), 0.92$ (d, $\left.J=7.1 \mathrm{~Hz}, 3 \mathrm{H}, \mathrm{H} 24 \mathrm{a}-\mathrm{CH}_{3}\right), 0.85$ (d, $\left.J=12.4 \mathrm{~Hz}, 1 \mathrm{H}, \mathrm{H} 18 \mathrm{~b}\right) .{ }^{13} \mathrm{C}$ NMR (100 MHz, $\left.\mathrm{CDCl}_{3}\right) \delta 181.0,173.6,162.8,162.8,161.7,159.2,139.5,138.1$, 137.9, 136.2, 135.0, 132.2, 127.7, 125.3, 124.7, 120.5, 118.2, 118.0, 116.7, 116.5, 98.7, 95.7, 94.8, 81.8, 81.1, 80.4, 79.2, 79.1, 73.8, 68.4, 68.2, 68.2, 67.7, 67.0, 65.7, 57.2, 56.6, 55.7, 45.7, 40.3, 39.7, 38.6, 36.7, 34.6, 34.3, 32.4, 31.4, 30.0, 27.0, 26.6, 26.5, 25.5, 20.2, 20.0, 18.3, 17.2, 16.6, 15.2 .

S-24: The ${ }^{1} \mathrm{H}-\mathrm{NMR}$ and ${ }^{13} \mathrm{C}-\mathrm{NMR}$ data of $\mathbf{6 c}$ :

${ }^{1} \mathrm{H}$ NMR (400 MHz, $\mathrm{CDCl}_{3}$ ) $\delta 10.87$ (d, $\left.J=9.9 \mathrm{~Hz}, 1 \mathrm{H}, \mathrm{CONHCS}\right), 9.37$ (s, 1H, CSNH), 7.78-7.66 (m, 1H, Ph), 7.45 (d, J=4.1 Hz, 2H, Ph), 7.38 (dd, $J=8.0,4.2 \mathrm{~Hz}$, 1H, Ph), 5.87 (d, $J=9.9$ Hz, 1H, H9), 5.81-5.64 (m, 3H, H10, H11, H23), 5.57-5.46 (m, 2H, H22, H1"), 5.40 (s, 2H, H3, H19), 5.13-5.04 (m, 1H, H4" ), 4.99 (dd, J= 10.9, $3.7 \mathrm{~Hz}, 1 \mathrm{H}, \mathrm{H} 15), 4.77$ (d, J=3.8 Hz, 1H, H1'), 4.73-4.58 (m, 2H, H8a), 4.27 (t, $J=$ $6.8 \mathrm{~Hz}, 1 \mathrm{H}, \mathrm{H} 5), 4.16$ (d, J = $\left.6.7 \mathrm{~Hz}, 1 \mathrm{H}, \mathrm{H} 5{ }^{\prime \prime}\right), 4.08$ (s, 1H, H7-OH), 3.97-3.90 (m, 2H, H6, H13), 3.89-3.74 (m, 3H, H17, H5', H3'), 3.61 (ddd, $J=12.0,8.7,4.6$ Hz, 1H, H3'), 3.50 (s, 3H, H3"'-OMe), 3.41 (s, 3H, H3'-OMe), 3.34-3.19 (m, 3H, H2, H25, H4'), 2.50 (dt, $J=10.3,6.1 \mathrm{~Hz}, 2 \mathrm{H}, \mathrm{H} 12, \mathrm{H} 5-\mathrm{OH}$ ), 2.38-2.18 (m, 4H, H16, H24, H2"a), 2.12 (dd, $\left.J=13.8,4.9 \mathrm{~Hz}, 1 \mathrm{H}, \mathrm{H} 2^{\prime} \mathrm{a}\right), 1.98$ (dd, $\left.J=12.1,4.8 \mathrm{~Hz}, 1 \mathrm{H}, \mathrm{H} 20 \mathrm{a}\right)$, 1.84 (s, 3H, H4a-CH $)$, 1.81-1.71 (m, 3H, H27a, H30a, H31a), 1.69-1.61 (m, 2H, H18a, H28a), 1.54 (d, J = 8.5 Hz, 3H, H26, H29), 1.46 (d, J = 11.9 Hz, 4H, H14a-CH H20b), 1.35-1.10 (m, 15H, H2'b, H27b, H28b, H30b, H31b, H2'b, H5'-Me, H5"-Me, $\left.\mathrm{H} 12 \mathrm{a}-\mathrm{CH}_{3}\right), 0.91$ (d, $\left.J=7.1 \mathrm{~Hz}, 3 \mathrm{H}, \mathrm{H} 24 \mathrm{a}-\mathrm{CH}_{3}\right), 0.84$ (d, J=12.2 Hz, $\left.1 \mathrm{H}, \mathrm{H} 18 \mathrm{~b}\right) .{ }^{13} \mathrm{C}$ NMR $\left(100 \mathrm{MHz}, \mathrm{CDCl}_{3}\right) \delta 180.7,173.6,165.8,139.5,138.0,137.8,136.1,135.0$, 
133.1, 132.1, 131.1, 130.8, 130.6, 127.7, 127.4, 124.7, 120.4, 118.2, 118.0, 98.6, 95.7, $94.8,81.8,81.0,80.3,79.2,79.1,77.3,73.8,68.4,68.2,68.2,67.7,67.0,65.7,57.2$, 56.6, 55.7, 45.7, 40.3, 39.7, 38.6, 36.7, 34.6, 34.3, 32.3, 31.4, 30.0, 26.9, 26.6, 26.5, 25.5, 20.2, 19.9, 18.3, 17.2, 16.6, 15.1 .

S-25: The ${ }^{1} \mathrm{H}-\mathrm{NMR}$ and ${ }^{13} \mathrm{C}-\mathrm{NMR}$ data of $\mathbf{6 d}$ :

${ }^{1} \mathrm{H}$ NMR $\left(400 \mathrm{MHz}, \mathrm{CDCl}_{3}\right) \delta 10.72(\mathrm{~d}, J=9.9 \mathrm{~Hz}, 1 \mathrm{H}, \mathrm{CONHCS}), 8.88(\mathrm{~s}, 1 \mathrm{H}$, CSNH), 7.82-7.72 (m, 1H, Ph), 7.71-7.57 (m, 3H, Ph), 5.92-5.85 (m, 1H, H9), 5.83-5.67 (m, 3H, H10, H11, H23), 5.58-5.48 (m, 2H, H22, H1"), 5.47-5.33 (m, 2H, H3, H19), 5.07 (dd, $\left.J=10.0,3.5 \mathrm{~Hz}, 1 \mathrm{H}, \mathrm{H} 4{ }^{\prime \prime}\right), 5.04-4.96$ (m, 1H, H15), 4.78 (d, $J=$ $\left.3.8 \mathrm{~Hz}, 1 \mathrm{H}, \mathrm{H1})^{\prime}\right), 4.73-4.62$ (m, 2H, H8a), 4.28 (d, $\left.J=6.6 \mathrm{~Hz}, 1 \mathrm{H}, \mathrm{H} 5\right), 4.18$ (q, $J=$ $\left.6.5 \mathrm{~Hz}, 1 \mathrm{H}, \mathrm{H} 5{ }^{\prime \prime}\right), 4.07$ (s, 1H, H7-OH), 3.96 (d, J = 6.4 Hz, 1H, H6), 3.94 (s, 1H, H13), 3.91-3.76 (m, 3H, H17, H5', H3'), 3.62 (ddd, $J=11.3,8.7,4.7$ Hz, 1H, H3'), 3.52 (s, 3H, H3"'-OMe), 3.42 (s, 3H, H3'-OMe), 3.34-3.22 (m, 3H, H2, H25, H4'), 2.59-2.48 (m, 1H, H12), 2.40 (d, $J=8.1 \mathrm{~Hz}, 1 \mathrm{H}, \mathrm{H} 5-\mathrm{OH}), 2.35-2.18$ (m, 4H, H16, H24, H2"a), 2.13 (dd, $J=13.7,4.9 \mathrm{~Hz}, 1 \mathrm{H}, \mathrm{H} 2^{\prime} \mathrm{a}$ ), 1.99 (dd, $J=11.8,4.7 \mathrm{~Hz}, 1 \mathrm{H}$, H20a), 1.86 (t, $\left.J=2.0 \mathrm{~Hz}, 3 \mathrm{H}, \mathrm{H} 4 \mathrm{a}-\mathrm{CH}_{3}\right), 1.83-1.72$ (m, 3H, H27a, H30a, H31a), 1.72-1.64 (m, 2H, H18a, H28a), 1.58-1.53 (m, 3H, H26, H29), 1.51-1.44 (m, 4H, $\left.\mathrm{H} 14 \mathrm{a}-\mathrm{CH}_{3}, \mathrm{H} 20 \mathrm{~b}\right), 1.37-1.11$ (m, 15H, H2'b, H27b, H28b, H30b, H31b, H2 b, H5'-Me, H5"-Me, H12a-CH $), 0.92$ (d, $\left.J=7.1 \mathrm{~Hz}, 3 \mathrm{H}, \mathrm{H} 24 \mathrm{a}-\mathrm{CH}_{3}\right), 0.88-0.82(\mathrm{~m}, 1 \mathrm{H}$, H18b). ${ }^{13} \mathrm{C}$ NMR (100 MHz, $\left.\mathrm{CDCl}_{3}\right) \delta 180.7,173.6,167.2,139.5,138.1,137.9,136.2$, 135.0, 132.8, 132.2, 131.4, 128.0, 127.7, 127.0, 126.9, 124.7, 120.5, 118.2, 118.0, 98.6, 95.7, 94.9, 81.8, 80.9, 80.4, 79.2, 79.1, 73.7, 68.4, 68.3, 68.2, 67.7, 67.0, 65.6, 57.2, 56.6, 55.8, 45.7, 40.3, 39.7, 38.6, 36.7, 34.6, 34.3, 32.3, 31.4, 30.0, 29.3, 27.2, $26.9,26.6,26.5,25.5,20.2,19.9,18.3,17.1,16.6,15.1$.

S-26: The ${ }^{1} \mathrm{H}-\mathrm{NMR}$ and ${ }^{13} \mathrm{C}-\mathrm{NMR}$ data of $\mathbf{6 e}$ :

${ }^{1} \mathrm{H}$ NMR (400 MHz, $\mathrm{CDCl}_{3}$ ) $\delta 11.12$ (d, $\left.J=9.9 \mathrm{~Hz}, 1 \mathrm{H}, \mathrm{CONHCS}\right), 9.01$ (s, 1H, CSNH), 7.68-7.60 (m, 2H, Ph), 7.45-7.34 (m, 2H, Ph), 5.90 (d, $J=10.2 \mathrm{~Hz}, 1 \mathrm{H}, \mathrm{H} 9)$, 5.83-5.67 (m, 3H, H10, H11, H23), 5.57-5.49 (m, 2H, H22, H1"), 5.48-5.36 (m, 2H, H3, H19), 5.09 (dd, $\left.J=10.0,3.6 \mathrm{~Hz}, 1 \mathrm{H}, \mathrm{H} 4^{\prime \prime}\right), 5.01$ (d, $\left.J=10.6 \mathrm{~Hz}, 1 \mathrm{H}, \mathrm{H} 15\right), 4.79$ (d, $\left.J=3.8 \mathrm{~Hz}, 1 \mathrm{H}, \mathrm{H1} 1^{\prime}\right), 4.69$ (t, $\left.J=2.9 \mathrm{~Hz}, 2 \mathrm{H}, \mathrm{H} 8 \mathrm{a}\right), 4.30$ (d, $\left.J=6.3 \mathrm{~Hz}, 1 \mathrm{H}, \mathrm{H} 5\right)$, 
4.23-4.14 (m, 1H, H5"), 4.04 (s, 1H, H7-OH), 3.98 (d, J = 6.2 Hz, 1H, H6), 3.95 (s, 1H, H13), 3.91-3.78 (m, 3H, H17, H5', H3"'), 3.66-3.58 (m, 1H, H3'), 3.52 (s, 3H, H3"-OMe), 3.43 (s, 3H, H3'-OMe), 3.33-3.22 (m, 3H, H2, H25, H4'), 2.53 (t, J = 7.4 Hz, 1H, H12), 2.42 (s, 3H, $\mathrm{CH}_{3}$ ), 2.37-2.20 (m, 5H, H5-OH, H16, H24, H2"a), 2.14 (dd, $\left.J=13.8,5.0 \mathrm{~Hz}, 1 \mathrm{H}, \mathrm{H} 2^{\prime} \mathrm{a}\right), 2.00$ (dd, $\left.J=12.2,4.8 \mathrm{~Hz}, 1 \mathrm{H}, \mathrm{H} 20 \mathrm{a}\right), 1.88$ (s, 3H,

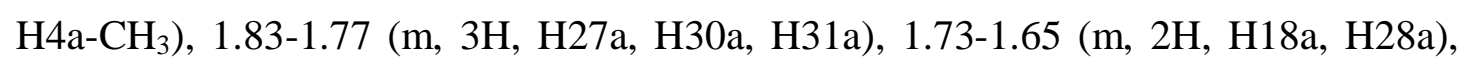
1.56 (s, 3H, H26, H29), 1.49 (d, $J=7.5$ Hz, 4H, H14a-CH, $\mathrm{H} 20 \mathrm{~b}$ ), 1.32-1.14 (m, 15H, H2'b, H27b, H28b, H30b, H31b, H2'b, H5'-Me, H5"'-Me, H12a-CH ${ }_{3}$ ), 0.93 (d, $\left.J=7.1 \mathrm{~Hz}, 3 \mathrm{H}, \mathrm{H} 24 \mathrm{a}-\mathrm{CH}_{3}\right), 0.88-0.83(\mathrm{~m}, 1 \mathrm{H}, \mathrm{H} 18 \mathrm{~b}) .{ }^{13} \mathrm{C} \mathrm{NMR}\left(100 \mathrm{MHz}, \mathrm{CDCl}_{3}\right) \delta$ 181.2, 173.6, 167.1, 139.5, 139.0, 138.1, 137.9, 136.2, 135.0, 134.2, 131.7, 128.9, 128.1, 127.7, 124.7, 124.6, 120.5, 118.2, 118.1, 98.6, 95.7, 94.8, 81.8, 81.0, 80.4, 79.2, 79.1, 73.8, 68.4, 68.2, 68.2, 67.7, 67.0, 65.7, 57.1, 56.6, 55.7, 45.7, 40.3, 39.7, 38.6, $36.7,34.6,34.3,32.3,31.4,30.0,26.9,26.6,26.5,25.5,21.3,20.2$, 19.9, 18.3, 17.2, 16.6, 15.1 .

S-27: The ${ }^{1} \mathrm{H}-\mathrm{NMR}$ and ${ }^{13} \mathrm{C}-\mathrm{NMR}$ data of $6 \mathbf{6}$ :

${ }^{1} \mathrm{H}$ NMR (400 MHz, $\mathrm{CDCl}_{3}$ ) $\delta 11.02$ (d, $\left.J=9.8 \mathrm{~Hz}, 1 \mathrm{H}, \mathrm{CONHCS}\right), 9.27$ (d, $J$ $=2.9 \mathrm{~Hz}, 1 \mathrm{H}, \mathrm{CSNH}), 8.16(\mathrm{~s}, 1 \mathrm{H}, \mathrm{Ph}), 8.04(\mathrm{~d}, J=7.9 \mathrm{~Hz}, 1 \mathrm{H}, \mathrm{Ph}), 7.86(\mathrm{~d}, J=7.7$ $\mathrm{Hz}, 1 \mathrm{H}, \mathrm{Ph}), 7.65$ (t, J = 7.8 Hz, 1H, Ph), 5.92-5.84 (m, 1H, H9), 5.82-5.64 (m, 3H, H10, H11, H23), 5.57-5.49 (m, 2H, H22, H1'), 5.41 (s, 2H, H3, H19), 5.07 (dd, J = 10.0, 3.5 Hz, 1H, H4"), 4.99 (dd, $J=10.9,3.7 \mathrm{~Hz}, 1 \mathrm{H}, \mathrm{H} 15$ ), 4.78 (d, $J=3.9 \mathrm{~Hz}, 1 \mathrm{H}$, H1') $4.72-4.59$ (m, 2H, H8a), 4.29 (t, $J=6.8 \mathrm{~Hz}, 1 \mathrm{H}, \mathrm{H} 5), 4.18$ (q, $J=6.5 \mathrm{~Hz}, 1 \mathrm{H}$, H5"), 4.07 (s, 1H, H7-OH), 3.95 (d, $J=6.3$ Hz, 1H, H6), 3.93 (s, 1H, H13), 3.91-3.76 (m, 3H, H17, H5', H3"), 3.61 (ddd, $J=11.2,8.6,4.7 \mathrm{~Hz}, 1 \mathrm{H}, \mathrm{H} 3$ '), 3.49 (s, 3H, H3"-OMe), 3.42 (s, 3H, H3'-OMe), 3.33-3.20 (m, 3H, H2, H25, H4'), 2.59-2.47 (m, 2H, H12, H5-OH), 2.36-2.20 (m, 4H, H16, H24, H2"a), 2.14 (dd, J = 13.8, 5.0 Hz, 1H, H2'a), 2.03-1.95 (m, 1H, H20a), 1.85 (s, 3H, H4a-CH ${ }_{3}$ ) 1.82-1.73 (m, 3H, H27a, H30a, H31a), 1.71-1.61 (m, 2H, H18a, H28a), 1.55 (d, J = 8.3 Hz, 3H, H26, H29), 1.47 (d, $J=9.6 \mathrm{~Hz}, 4 \mathrm{H}, \mathrm{H} 14 \mathrm{a}-\mathrm{CH}_{3}, \mathrm{H} 20 \mathrm{~b}$ ), 1.37-1.09 (m, 15H, H2'b, H27b, H28b, H30b, H31b, H2'b, H5'-Me, H5"-Me, H12a-CH3), 0.91 (d, J = $7.1 \mathrm{~Hz}, 3 \mathrm{H}$, $\left.\mathrm{H} 24 \mathrm{a}-\mathrm{CH}_{3}\right), 0.84(\mathrm{~d}, J=12.2 \mathrm{~Hz}, 1 \mathrm{H}, \mathrm{H} 18 \mathrm{~b}) .{ }^{13} \mathrm{C} \mathrm{NMR}\left(100 \mathrm{MHz}, \mathrm{CDCl}_{3}\right) \delta 180.9$, 
173.6, 165.5, 139.5, 138.0, 137.9, 136.2, 135.0, 132.8, 131.9, 131.6, 130.5, 129.7, 127.7, 124.9, 124.9, 124.7, 121.9, 120.5, 118.2, 118.1, 98.6, 95.7, 94.8, 81.8, 81.1, 80.4, 79.2, 79.1, 73.8, 68.4, 68.2, 68.2, 67.7, 67.0, 65.6, 57.2, 56.6, 55.9, 45.7, 40.3, 39.7, 38.6, 36.7, 34.6, 34.3, 32.3, 31.4, 30.0, 26.9, 26.6, 26.5, 25.5, 20.2, 19.9, 18.3, $17.2,16.6,15.1$.

S-28: The ${ }^{1} \mathrm{H}-\mathrm{NMR}$ and ${ }^{13} \mathrm{C}-\mathrm{NMR}$ data of $\mathbf{6 g}$ :

${ }^{1} \mathrm{H}$ NMR (400 MHz, $\mathrm{CDCl}_{3}$ ) $\delta 11.06$ (d, $\left.J=9.8 \mathrm{~Hz}, 1 \mathrm{H}, \mathrm{CONHCS}\right), 9.06$ (s, 1H, CSNH), 7.94-7.84 (m, 2H, Ph), 7.19 (t, $J=8.5 \mathrm{~Hz}, 2 \mathrm{H}, \mathrm{Ph}), 5.93-5.86$ (m, 1H, H9), 5.82-5.68 (m, 3H, H10, H11, H23), 5.57-5.48 (m, 2H, H22, H1'), 5.47-5.31 (m, 2H, H3, H19), 5.08 (dd, $\left.J=10.3,3.6 \mathrm{~Hz}, 1 \mathrm{H}, \mathrm{H} 4{ }^{\prime \prime}\right), 5.01$ (d, $\left.J=10.6 \mathrm{~Hz}, 1 \mathrm{H}, \mathrm{H} 15\right), 4.79$ (d, $\left.J=3.8 \mathrm{~Hz}, 1 \mathrm{H}, \mathrm{H1} 1^{\prime}\right), 4.68(\mathrm{t}, J=3.1 \mathrm{~Hz}, 2 \mathrm{H}, \mathrm{H} 8 \mathrm{a}), 4.30$ (d, $\left.J=6.2 \mathrm{~Hz}, 1 \mathrm{H}, \mathrm{H} 5\right)$, 4.17 (p, $\left.J=6.1 \mathrm{~Hz}, 1 \mathrm{H}, \mathrm{H} 5^{\prime \prime}\right), 4.06$ (s, 1H, H7-OH), 3.97 (d, $\left.J=6.2 \mathrm{~Hz}, 1 \mathrm{H}, \mathrm{H} 6\right)$, 3.94 (s, 1H, H13), 3.91-3.75 (m, 3H, H17, H5', H3"), 3.62 (ddd, $J=11.2,8.6,4.8 \mathrm{~Hz}$, 1H, H3), 3.51 (s, 3H, H3"'-OMe), 3.43 (s, 3H, H3'-OMe), 3.34-3.20 (m, 3H, H2, H25, H4'), 2.53 (t, $J=7.7$ Hz, 1H, H12), 2.45-2.20 (m, 5H, H5-OH, H16, H24, H2"a), 2.14 (dd, $\left.J=13.6,5.0 \mathrm{~Hz}, 1 \mathrm{H}, \mathrm{H} 2^{\prime} \mathrm{a}\right), 2.00$ (dd, $\left.J=12.3,5.0 \mathrm{~Hz}, 1 \mathrm{H}, \mathrm{H} 20 \mathrm{a}\right), 1.87$ (s, 3H, H4a-CH $_{3}$ 1.83-1.75 (m, 3H, H27a, H30a, H31a), 1.73-1.67 (m, 2H, H18a, H28a), 1.56 (d, $J=8.2 \mathrm{~Hz}, 3 \mathrm{H}, \mathrm{H} 26, \mathrm{H} 29), 1.49$ (d, $J=8.5 \mathrm{~Hz}, 4 \mathrm{H}, \mathrm{H} 14 \mathrm{a}-\mathrm{CH}_{3}, \mathrm{H} 20 \mathrm{~b}$ ), 1.31-1.13 (m, 15H, H2"b, H27b, H28b, H30b, H31b, H2'b, H5'-Me, H5"-Me, $\left.\mathrm{H} 12 \mathrm{a}-\mathrm{CH}_{3}\right), 0.92\left(\mathrm{~d}, J=7.2 \mathrm{~Hz}, 3 \mathrm{H}, \mathrm{H} 24 \mathrm{a}-\mathrm{CH}_{3}\right), 0.86(\mathrm{~d}, J=12.5 \mathrm{~Hz}, 1 \mathrm{H}, \mathrm{H} 18 \mathrm{~b}) .{ }^{13} \mathrm{C}$ NMR $\left(100 \mathrm{MHz}, \mathrm{CDCl}_{3}\right) \delta 181.2,173.5,167.1,165.9,164.5,139.4,138.0,137.8$, 136.2, 135.0, 130.3, 130.2, 128.0, 128.0, 127.7, 124.7, 120.5, 118.2, 118.1, 116.4, 116.1, 98.6, 95.7, 94.8, 81.8, 81.1, 80.4, 79.2, 73.8, 68.3, 68.2, 68.2, 67.7, 67.0, 65.7, 57.1, 56.6, 55.7, 45.7, 40.3, 39.7, 38.6, 36.6, 34.6, 34.3, 32.3, 31.4, 30.0, 26.9, 26.6, $26.5,25.5,20.2,19.9,18.3,17.2,16.6,15.1$.

S-29: The ${ }^{1} \mathrm{H}-\mathrm{NMR}$ and ${ }^{13} \mathrm{C}-\mathrm{NMR}$ data of $\mathbf{6 h}$ :

${ }^{1} \mathrm{H}$ NMR (400 MHz, $\mathrm{CDCl}_{3}$ ) $\delta 11.03$ (d, $\left.J=9.8 \mathrm{~Hz}, 1 \mathrm{H}, \mathrm{CONHCS}\right), 9.01$ (s, 1H, CSNH), $7.80(\mathrm{~d}, J=8.6 \mathrm{~Hz}, 2 \mathrm{H}, \mathrm{Ph}), 7.49$ (d, $J=8.6 \mathrm{~Hz}, 2 \mathrm{H}, \mathrm{Ph}), 5.93-5.85$ (m, 1H, H9), 5.83-5.67 (m, 3H, H10, H11, H23), 5.59-5.48 (m, 2H, H22, H1"), 5.48-5.32 (m, 2H, H3, H19), 5.08 (dd, $\left.J=9.8,3.5 \mathrm{~Hz}, 1 \mathrm{H}, \mathrm{H} 4^{\prime \prime}\right), 5.01$ (d, $J=10.6 \mathrm{~Hz}, 1 \mathrm{H}, \mathrm{H} 15$ ), 
$4.79\left(\mathrm{~d}, J=3.8 \mathrm{~Hz}, 1 \mathrm{H}, \mathrm{H} 1^{\prime}\right), 4.74-4.62$ (m, 2H, H8a), 4.30 (d, $\left.J=6.2 \mathrm{~Hz}, 1 \mathrm{H}, \mathrm{H} 5\right)$, $4.22-4.14$ (m, 1H, H5"), 4.05 (s, 1H, H7-OH), 3.97 (d, J=6.3 Hz, 1H, H6), 3.94 (s, 1H, H13), 3.91-3.78 (m, 3H, H17, H5', H3"), 3.62 (ddd, $J=11.1,8.6,4.8 \mathrm{~Hz}, 1 \mathrm{H}$, H3'), 3.51 (s, 3H, H3'"-OMe), 3.43 (s, 3H, H3'-OMe), 3.33-3.22 (m, 3H, H2, H25, H4'), 2.53 (t, $J=7.0 \mathrm{~Hz}, 1 \mathrm{H}, \mathrm{H} 12), 2.37$ (s, 1H, H5-OH), 2.33-2.20 (m, 4H, H16, H24, H2"a), 2.14 (dd, $\left.J=13.7,5.0 \mathrm{~Hz}, 1 \mathrm{H}, \mathrm{H} 2^{\prime} \mathrm{a}\right), 2.03-1.96$ (m, 1H, H20a), 1.87 (s, 3H,

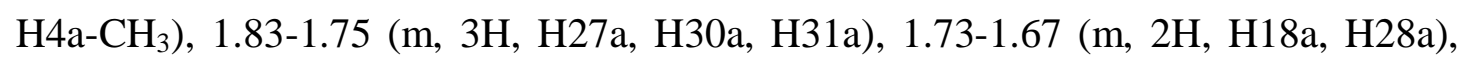
$1.56(\mathrm{~d}, J=8.3 \mathrm{~Hz}, 3 \mathrm{H}, \mathrm{H} 26, \mathrm{H} 29), 1.49$ (d, $\left.J=7.6 \mathrm{~Hz}, 4 \mathrm{H}, \mathrm{H} 14 \mathrm{a}-\mathrm{CH}_{3}, \mathrm{H} 20 \mathrm{~b}\right)$, 1.35-1.13 (m, 15H, H2'b, H27b, H28b, H30b, H31b, H2'b, H5'-Me, H5"-Me, $\left.\mathrm{H} 12 \mathrm{a}-\mathrm{CH}_{3}\right), 0.93$ (d, $\left.J=7.1 \mathrm{~Hz}, 3 \mathrm{H}, \mathrm{H} 24 \mathrm{a}-\mathrm{CH}_{3}\right), 0.88-0.82(\mathrm{~m}, 1 \mathrm{H}, \mathrm{H} 18 \mathrm{~b}) .{ }^{13} \mathrm{C} \mathrm{NMR}$ $\left(100 \mathrm{MHz}, \mathrm{CDCl}_{3}\right) \delta 181.1,173.5,165.9,140.0,139.5,138.0,137.8,136.2,135.0$, 130.2, 129.3(2-C), 129.0(2-C), 127.7, 124.7, 120.5, 118.2, 118.1, 98.6, 95.7, 94.8, 81.8, 81.1, 80.4, 79.2, 77.3, 73.8, 68.3, 68.2, 68.2, 67.7, 67.0, 65.7, 57.2, 56.6, 55.7, $45.7,40.3,39.7,38.6,36.6,34.6,34.3,32.3,31.4,30.0,26.9,26.6,26.5,25.5,20.2$, $19.9,18.3,17.2,16.6,15.1$.

S-30: The ${ }^{1} \mathrm{H}-\mathrm{NMR}$ and ${ }^{13} \mathrm{C}-\mathrm{NMR}$ data of $6 \mathbf{i}$ :

${ }^{1} \mathrm{H}$ NMR (400 MHz, $\left.\mathrm{CDCl}_{3}\right) \delta 11.02$ (d, $\left.J=9.8 \mathrm{~Hz}, 1 \mathrm{H}, \mathrm{CONHCS}\right), 9.01$ (s, 1H, CSNH), 7.73 (d, $J=8.7 \mathrm{~Hz}, 2 \mathrm{H}, \mathrm{Ph}), 7.66$ (d, $J=8.6 \mathrm{~Hz}, 2 \mathrm{H}, \mathrm{Ph}), 5.91-5.87$ (m, 1H, H9), 5.83-5.67 (m, 3H, H10, H11, H23), 5.57-5.48 (m, 2H, H22, H1"), 5.48-5.35 (m, 2H, H3, H19), 5.10-5.04 (m, 1H, H4"'), 5.01 (d, $J=10.5 \mathrm{~Hz}, 1 \mathrm{H}, \mathrm{H} 15), 4.79$ (d, $J=$ $3.8 \mathrm{~Hz}, 1 \mathrm{H}, \mathrm{H1}$ ), $4.74-4.63$ (m, 2H, H8a), 4.30 (t, $J=6.7 \mathrm{~Hz}, 1 \mathrm{H}, \mathrm{H} 5), 4.18$ (q, $J=$ $\left.6.4 \mathrm{~Hz}, 1 \mathrm{H}, \mathrm{H} 5{ }^{\prime \prime}\right), 4.05$ (s, 1H, H7-OH), 3.97 (d, J = 6.2 Hz, 1H, H6), 3.94 (s, 1H, H13), 3.92-3.77 (m, 3H, H17, H5', H3"'), 3.62 (ddd, $J=11.2,8.6,4.8$ Hz, 1H, H3'), 3.51 (s, 3H, H3"'-OMe), 3.43 (s, 3H, H3'-OMe), 3.35-3.21 (m, 3H, H2, H25, H4'), $2.53(\mathrm{t}, J=7.3 \mathrm{~Hz}, 1 \mathrm{H}, \mathrm{H} 12), 2.38$ (d, $J=8.0 \mathrm{~Hz}, 1 \mathrm{H}, \mathrm{H} 5-\mathrm{OH}), 2.34-2.20$ (m, 4H, H16, H24, H2"a), 2.14 (dd, $\left.J=13.7,5.0 \mathrm{~Hz}, 1 \mathrm{H}, \mathrm{H} 2{ }^{\prime} \mathrm{a}\right), 2.03-1.97$ (m, 1H, H20a), 1.87 (s, 3H, H4a-CH $)$, 1.82-1.73 (m, 3H, H27a, H30a, H31a), 1.72-1.67 (m, 2H, H18a, H28a), 1.56 (d, $J=8.4$ Hz, 3H, H26, H29), 1.49 (d, $J=7.5 \mathrm{~Hz}, 4 \mathrm{H}, \mathrm{H} 14 \mathrm{a}-\mathrm{CH}_{3}$, H20b), 1.34-1.11 (m, 15H, H2'b, H27b, H28b, H30b, H31b, H2'b, H5'-Me, H5"-Me, $\left.\mathrm{H} 12 \mathrm{a}-\mathrm{CH}_{3}\right), 0.93\left(\mathrm{~d}, J=7.1 \mathrm{~Hz}, 3 \mathrm{H}, \mathrm{H} 24 \mathrm{a}-\mathrm{CH}_{3}\right), 0.86(\mathrm{~d}, J=13.7 \mathrm{~Hz}, 1 \mathrm{H}, \mathrm{H} 18 \mathrm{~b}) .{ }^{13} \mathrm{C}$ 
NMR $\left(100 \mathrm{MHz}, \mathrm{CDCl}_{3}\right) \delta 181.0,173.6,166.0,139.5,138.0,137.8,136.2,135.0$, 132.3(2-C), 130.6, 129.1(2-C), 128.6, 127.7, 124.7, 120.5, 118.2, 118.1, 98.6, 95.7, 94.8, 81.8, 81.1, 80.4, 79.2, 79.2, 73.8, 68.4, 68.2, 68.2, 67.7, 67.0, 65.7, 57.2, 56.6, 55.8, 45.7, 40.3, 39.7, 38.6, 36.7, 34.6, 34.3, 32.3, 31.4, 30.0, 29.6, 27.0, 26.6, 26.5, $25.5,20.2,19.9,18.3,17.2,16.6,15.1$.

S-31: The ${ }^{1} \mathrm{H}-\mathrm{NMR}$ and ${ }^{13} \mathrm{C}-\mathrm{NMR}$ data of $\mathbf{6 j}$ :

${ }^{1} \mathrm{H}$ NMR (400 MHz, $\mathrm{CDCl}_{3}$ ) $\delta 11.17$ (d, $\left.J=9.8 \mathrm{~Hz}, 1 \mathrm{H}, \mathrm{CONHCS}\right), 8.97$ (s, 1H, CSNH), 7.82 (d, J=8.9 Hz, 2H, Ph), 6.98 (d, $J=8.9 \mathrm{~Hz}, 2 \mathrm{H}, \mathrm{Ph}), 5.89$ (d, $J=10.0$ Hz, 1H, H9), 5.82-5.67 (m, 3H, H10, H11, H23), 5.57-5.50 (m, 2H, H22, H1"), 5.48-5.35 (m, 2H, H3, H19), 5.13-5.05 (m, 1H, H4"'), 5.01 (d, J = 10.7 Hz, 1H, H15), $4.79\left(\mathrm{~d}, J=4.0 \mathrm{~Hz}, 1 \mathrm{H}, \mathrm{H} 1^{\prime}\right), 4.69$ (t, $\left.J=3.0 \mathrm{~Hz}, 2 \mathrm{H}, \mathrm{H} 8 \mathrm{a}\right), 4.30$ (t, $J=7.0 \mathrm{~Hz}, 1 \mathrm{H}$, H5), 4.21-4.14 (m, 1H, H5"), 4.04 (s, 1H, H7-OH), 3.98 (d, J=6.2 Hz, 1H, H6), 3.94 (s, 1H, H13), 3.92-3.77 (m, 3H, H17, H5', H3'), 3.88 (s, 3H, $\left.\mathrm{OCH}_{3}\right), 3.67-3.58$ (m, 1H, H3'), 3.52 (s, 3H, H3"-OMe), 3.43 (s, 3H, H3'-OMe), 3.34-3.21 (m, 3H, H2, H25, H4'), 2.53 (t, $J=7.8 \mathrm{~Hz}, 1 \mathrm{H}, \mathrm{H} 12), 2.37$ (d, $J=7.9 \mathrm{~Hz}, 1 \mathrm{H}, \mathrm{H} 5-\mathrm{OH}), 2.33-2.20$ (m, 4H, H16, H24, H2"'a), 2.13 (dd, $J=13.6,4.9$ Hz, 1H, H2'a), 2.03-1.97 (m, 1H, H20a), 1.88 (s, 3H, H4a-CH $)$, 1.83-1.74 (m, 3H, H27a, H30a, H31a), 1.72-1.65 (m, 2H, H18a, H28a), 1.56 (t, $J=5.2 \mathrm{~Hz}, 3 \mathrm{H}, \mathrm{H} 26, \mathrm{H} 29), 1.49$ (d, $J=7.6 \mathrm{~Hz}, 4 \mathrm{H}, \mathrm{H} 14 \mathrm{a}-\mathrm{CH}_{3}$, H20b), 1.36-1.12 (m, 15H, H2'b, H27b, H28b, H30b, H31b, H2'b, H5'-Me, H5"-Me, $\left.\mathrm{H} 12 \mathrm{a}-\mathrm{CH}_{3}\right), 0.93\left(\mathrm{~d}, J=7.1 \mathrm{~Hz}, 3 \mathrm{H}, \mathrm{H} 24 \mathrm{a}-\mathrm{CH}_{3}\right), 0.88-0.83$ (m, 1H, H18b). ${ }^{13} \mathrm{C}$ NMR $\left(100 \mathrm{MHz}, \mathrm{CDCl}_{3}\right) \delta 181.4,173.4,166.4,163.7,139.5,138.0,137.7,136.1,135.0$, 129.7(2-C), 127.7, 124.7, 123.7, 120.4, 118.2, 118.1, 114.2(2-C), 98.6, 95.7, 94.8, 81.8, 81.0, 80.4, 79.2(2-C), 77.3, 73.8, 68.3, 68.2, 68.2, 67.7, 67.0, 65.7, 57.1, 56.6, 55.6, 55.5, 45.7, 40.3, 39.7, 38.6, 36.6, 34.6, 34.3, 32.3, 31.4, 30.0, 26.9, 26.6, 26.4, 25.5, 20.2, 19.9, 18.3, 17.2, 16.6, 15.1 .

S-32: The ${ }^{1} \mathrm{H}-\mathrm{NMR}$ and ${ }^{13} \mathrm{C}-\mathrm{NMR}$ data of $\mathbf{6 k}$ :

${ }^{1} \mathrm{H}$ NMR $\left(400 \mathrm{MHz}, \mathrm{CDCl}_{3}\right) \delta 10.98(\mathrm{~d}, J=9.9 \mathrm{~Hz}, 1 \mathrm{H}, \mathrm{CONHCS}), 9.11(\mathrm{~s}, 1 \mathrm{H}$, CSNH), 7.99 (d, $J=8.2 \mathrm{~Hz}, 2 \mathrm{H}, \mathrm{Ph}), 7.79$ (d, $J=8.1 \mathrm{~Hz}, 2 \mathrm{H}, \mathrm{Ph}), 5.93-5.85$ (m, 1H, H9), 5.83-5.68 (m, 3H, H10, H11, H23), 5.58-5.50 (m, 2H, H22, H1"), 5.48-5.32 (m, 2H, H3, H19), 5.08 (dd, $\left.J=9.5,3.4 \mathrm{~Hz}, 1 \mathrm{H}, \mathrm{H} 4{ }^{\prime \prime}\right), 5.01$ (d, $\left.J=10.7 \mathrm{~Hz}, 1 \mathrm{H}, \mathrm{H} 15\right)$, 
$4.79\left(\mathrm{~d}, J=3.8 \mathrm{~Hz}, 1 \mathrm{H}, \mathrm{H} 1^{\prime}\right), 4.69$ (t, $\left.J=3.0 \mathrm{~Hz}, 2 \mathrm{H}, \mathrm{H} 8 \mathrm{a}\right), 4.30$ (t, $J=7.0 \mathrm{~Hz}, 1 \mathrm{H}$, H5), 4.20 (q, $\left.J=6.5 \mathrm{~Hz}, 1 \mathrm{H}, \mathrm{H} 5^{\prime \prime}\right), 4.05$ (s, 1H, H7-OH), 3.97 (d, J=6.2 Hz, 1H, H6), 3.95 (s, 1H, H13), 3.93-3.79 (m, 3H, H17, H5', H3"'), 3.63 (td, $J=10.2,8.4,4.6 \mathrm{~Hz}$, 1H, H3'), 3.52 (s, 3H, H3"'-OMe), 3.44 (s, 3H, H3'-OMe), 3.35-3.22 (m, 3H, H2, H25, H4), 2.53 (t, $J=7.6 \mathrm{~Hz}, 1 \mathrm{H}, \mathrm{H} 12), 2.38$ (d, $J=8.1 \mathrm{~Hz}, 1 \mathrm{H}, \mathrm{H} 5-\mathrm{OH}), 2.34-2.19$ (m, 4H, H16, H24, H2"a), 2.15 (dd, $\left.J=13.8,4.9 \mathrm{~Hz}, 1 \mathrm{H}, \mathrm{H} 2{ }^{\prime} \mathrm{a}\right), 2.01$ (dd, $J=12.0,4.8$ Hz, 1H, H20a), 1.88 (s, 3H, H4a-CH $)$ 1.83-1.75 (m, 3H, H27a, H30a, H31a), 1.73-1.67 (m, 2H, H18a, H28a), 1.56 (d, $J=8.5$ Hz, 3H, H26, H29), 1.49 (d, $J=8.2$ Hz, 4H, H14a-CH3, $\mathrm{H} 20 \mathrm{~b}$ ), 1.37-1.13 (m, 15H, H2"'b, H27b, H28b, H30b, H31b, H2'b, H5'-Me, H5"'-Me, H12a-CH $), 0.93$ (d, $\left.J=7.1 \mathrm{~Hz}, 3 \mathrm{H}, \mathrm{H} 24 \mathrm{a}-\mathrm{CH}_{3}\right), 0.86$ (d, $J=$ $12.4 \mathrm{~Hz}, 1 \mathrm{H}, \mathrm{H} 18 \mathrm{~b}) .{ }^{13} \mathrm{C} \mathrm{NMR}\left(100 \mathrm{MHz}, \mathrm{CDCl}_{3}\right) \delta 180.9,173.5,165.7,139.5,138.0$, 137.8, 136.2, 135.2, 135.0, 128.1(2-C), 127.7, 126.0, 126.0, 124.7,121.9, 120.5, 118.2, 118.1, 98.6, 95.7, 94.8, 81.8, 81.1, 80.4, 79.2, 79.2, 73.8, 68.3, 68.2, 68.2, 67.7, 67.0, 65.6, 57.2, 56.6, 55.8, 45.7, 40.3, 39.7, 38.6, 36.7, 34.6, 34.3, 32.3, 31.4, 30.0, 26.9, $26.6,26.5,25.5,20.2,19.9,18.3,17.2,16.6,15.1$.

S-33: The ${ }^{1} \mathrm{H}-\mathrm{NMR}$ and ${ }^{13} \mathrm{C}-\mathrm{NMR}$ data of $6 \mathbf{0}$

${ }^{1} \mathrm{H}$ NMR $\left(400 \mathrm{MHz}, \mathrm{CDCl}_{3}\right) \delta 10.82(\mathrm{~d}, J=9.8 \mathrm{~Hz}, 1 \mathrm{H}, \mathrm{CONHCS}), 8.79(\mathrm{~d}, J=$ $4.1 \mathrm{~Hz}, 1 \mathrm{H}, \mathrm{CSNH}), 5.89$ (d, J = 9.9 Hz, 1H, H9), 5.82-5.65 (m, 3H, H10, H11, H23), 5.54 (dd, $J=9.9,2.4 \mathrm{~Hz}, 1 \mathrm{H}, \mathrm{H} 22), 5.49-5.30$ (m, 3H, H1", H3, H19), 5.01 (dd, $J=$ 10.5, 4.2 Hz, 2H, H4", H15), 4.80 (d, J = 3.7 Hz, 1H, H1'), 4.68 (t, $J=2.9 \mathrm{~Hz}, 2 \mathrm{H}$, H8a), 4.30 (d, $J=6.3 \mathrm{~Hz}, 1 \mathrm{H}, \mathrm{H} 5), 4.12$ (t, $\left.J=6.7 \mathrm{~Hz}, 1 \mathrm{H}, \mathrm{H} 5^{\prime \prime}\right), 4.05$ (s, 1H, H7-OH), 3.97 (d, J = 6.4 Hz, 1H, H6), 3.93 (s, 1H, H13), 3.90-3.70 (m, 3H, H17, H5', H3"), 3.65-3.55 (m, 1H, H3'), 3.48 (s, 3H, H3"-OMe), 3.41 (s, 3H, H3'-OMe), 3.34-3.12 (m, 3H, H2, H25, H4'), 2.58-2.48 (m, 1H, H12), 2.39 (s, 1H, H5-OH), 2.37-2.18 (m, 4H, H16, H24, H2"a), 2.14 (s, 3H, $\mathrm{CH}_{3}$ ), 2.07-1.95 (m, 2H, H2'a, H20a), 1.87 (s, 3H, H4a-CH $), 1.83-1.73$ (m, 3H, H27a, H30a, H31a), 1.73-1.66 (m, 2H, H18a, H28a), 1.55 (s, 3H, H26, H29), 1.48 (d, J = 8.0 Hz, 4H, H14a-CH, H2Ob), 1.32-1.12 (m, 15H, H2'b, H27b, H28b, H30b, H31b, H2'b, H5'-Me, H5"-Me, $\left.\mathrm{H} 12 \mathrm{a}-\mathrm{CH}_{3}\right), 0.92\left(\mathrm{~d}, J=7.1 \mathrm{~Hz}, 3 \mathrm{H}, \mathrm{H} 24 \mathrm{a}-\mathrm{CH}_{3}\right), 0.85$ (d, $\left.J=12.1 \mathrm{~Hz}, 1 \mathrm{H}, \mathrm{H} 18 \mathrm{~b}\right) .{ }^{13} \mathrm{C}$ NMR $\left(100 \mathrm{MHz}, \mathrm{CDCl}_{3}\right) \delta 181.0,173.6,171.1,139.4,138.0,137.8,136.2,135.0$, 
$127.7,124.7,120.5,118.2,118.1,98.5,95.7,94.8,81.8,80.9,80.4,79.2(2-\mathrm{C}), 73.7$, 68.4, 68.3, 68.2, 67.7, 67.0, 65.6, 57.1, 56.6, 55.4, 45.7, 40.3, 39.7, 38.6, 36.6, 34.6, $34.3,32.2,31.4,30.0,26.9,26.6,26.5,25.5,24.3,20.2,19.9,18.3,17.7,17.1,16.6$, 15.1 .

S-34: The ${ }^{1} \mathrm{H}-\mathrm{NMR}$ and ${ }^{13} \mathrm{C}-\mathrm{NMR}$ data of $\mathbf{6 m}$ :

${ }^{1} \mathrm{H}$ NMR $\left(400 \mathrm{MHz}, \mathrm{CDCl}_{3}\right) \delta 10.86(\mathrm{~d}, J=9.9 \mathrm{~Hz}, 1 \mathrm{H}, \mathrm{CONHCS}), 8.66(\mathrm{~s}, 1 \mathrm{H}$, CSNH), 5.92-5.85 (m, 1H, H9), 5.83-5.68 (m, 3H, H10, H11, H23), 5.54 (dd, $J=9.9$, $2.5 \mathrm{~Hz}, 1 \mathrm{H}, \mathrm{H} 22), 5.49-5.36$ (m, 3H, H1" $, \mathrm{H} 3, \mathrm{H} 19), 5.01$ (td, $J=9.8,3.5 \mathrm{~Hz}, 2 \mathrm{H}$, H4", H15), 4.78 (d, J = 3.9 Hz, 1H, H1'), 4.75-4.62 (m, 2H, H8a), 4.30 (s, 1H, H5), $4.13\left(\mathrm{~d}, J=6.8,6.4 \mathrm{~Hz}, 1 \mathrm{H}, \mathrm{H} 5^{\prime \prime}\right), 4.06$ (s, 1H, H7-OH), 3.97 (d, J=6.2 Hz, 1H, H6), 3.93 (s, 1H, H13), 3.89-3.73 (m, 3H, H17, H5', H3'), 3.60 (ddd, $J=11.4,8.6,4.8 \mathrm{~Hz}$, 1H, H3'), 3.48 (s, 3H, H3"'-OMe), 3.41 (s, 3H, H3'-OMe), 3.34-3.20 (m, 3H, H2, H25, H4'), 2.57-2.50 (m, 1H, H12), 2.42-2.18 (m, 7H, H5-OH, H16, H24, H2"'a, $\underline{\mathrm{CH}_{2}} \mathrm{CH}_{3}$ ), $2.09\left(\mathrm{dd}, J=13.6,4.9 \mathrm{~Hz}, 1 \mathrm{H}, \mathrm{H} 2^{\prime} \mathrm{a}\right), 2.00$ (dd, $\left.J=12.5,5.3 \mathrm{~Hz}, 1 \mathrm{H}, \mathrm{H} 20 \mathrm{a}\right), 1.87$ (s, 3H, H4a-CH ) $^{1.83-1.74 ~(m, ~ 3 H, ~ H 27 a, ~ H 30 a, ~ H 31 a), ~ 1.73-1.66 ~(s, ~ 2 H, ~ H 18 a, ~ H 28 a), ~}$ $1.56(\mathrm{~d}, J=7.8 \mathrm{~Hz}, 3 \mathrm{H}, \mathrm{H} 26, \mathrm{H} 29), 1.46$ (d, $\left.J=29.3 \mathrm{~Hz}, 4 \mathrm{H}, \mathrm{H} 14 \mathrm{a}-\mathrm{CH}_{3}, \mathrm{H} 20 \mathrm{~b}\right)$, 1.32-1.12 (m, 15H, H2"b, H27b, H28b, H30b, H31b, H2'b, H5'-Me, H5"-Me, $\left.\mathrm{H} 12 \mathrm{a}-\mathrm{CH}_{3}\right), 0.92\left(\mathrm{~d}, J=7.1 \mathrm{~Hz}, 3 \mathrm{H}, \mathrm{H} 24 \mathrm{a}-\mathrm{CH}_{3}\right), 0.89-0.82(\mathrm{~m}, 1 \mathrm{H}, \mathrm{H} 18 \mathrm{~b}) .{ }^{13} \mathrm{C} \mathrm{NMR}$ $\left(100 \mathrm{MHz}, \mathrm{CDCl}_{3}\right) \delta 181.0,174.2,173.7,139.5,138.1,137.9,136.2,135.0,127.7$, 124.7, 120.5, 118.2, 118.1, 98.6, 95.7, 94.8, 81.8, 80.9, 80.4, 79.2, 79.1, 73.7, 68.4, 68.2, 68.1, 67.7, 67.0, 65.6, 57.1, 56.6, 55.5, 45.7, 40.3, 39.7, 38.6, 36.7, 34.6, 34.3, $31.9,31.4,30.4,30.0,29.3,27.2,27.0,26.6,25.5,20.2,20.0,18.3,17.1,16.6,15.1$, 8.4 .

S-35: The ${ }^{1} \mathrm{H}-\mathrm{NMR}$ and ${ }^{13} \mathrm{C}-\mathrm{NMR}$ data of $\mathbf{6 n}$ :

${ }^{1} \mathrm{H}$ NMR $\left(400 \mathrm{MHz}, \mathrm{CDCl}_{3}\right) \delta 10.86(\mathrm{~d}, J=9.9 \mathrm{~Hz}, 1 \mathrm{H}, \mathrm{CONHCS}), 8.55$ (s, 1H, CSNH), 5.92-5.86 (m, 1H, H9), 5.82-5.67 (m, 3H, H10, H11, H23), 5.54 (dd, J = 9.9, $2.6 \mathrm{~Hz}, 1 \mathrm{H}, \mathrm{H} 22), 5.50-5.34$ (m, 3H, H1", H3, H19), 5.01 (t, $J=7.0 \mathrm{~Hz}, 2 \mathrm{H}, \mathrm{H} 4{ }^{\prime \prime}$, H15), 4.78 (d, $\left.J=3.7 \mathrm{~Hz}, 1 \mathrm{H}, \mathrm{H} 1^{\prime}\right), 4.68$ (t, $\left.J=2.9 \mathrm{~Hz}, 2 \mathrm{H}, \mathrm{H} 8 \mathrm{a}\right), 4.30$ (t, $J=7.1 \mathrm{~Hz}$, 1H, H5), 4.17-4.10 (m, 1H, H5"), 4.04 (s, 1H, H7-OH), 3.97 (d, J = 6.2 Hz, 1H, H6), 3.94 (s, 1H, H13), 3.91-3.73 (m, 3H, H17, H5', H3"), 3.61 (ddd, $J=11.1,8.4,4.7$ Hz, 
1H, H3'), 3.49 (s, 3H, H3"-OMe), 3.42 (s, 3H, H3'-OMe), 3.34-3.18 (m, 3H, H2, H25, H4'), 2.58-2.48 (m, 1H, H12), 2.37 (d, $J=8.1 \mathrm{~Hz}, 1 \mathrm{H}, \mathrm{H} 5-\mathrm{OH}), 2.33-2.19$ (m, 6H, H16, H24, H2"a, $\underline{\mathrm{CH}_{2}} \mathrm{CH}_{2} \mathrm{CH}_{3}$ ), 2.13-1.96 (m, 2H, H2'a, H20a), 1.87 (t, J = 2.0 Hz,

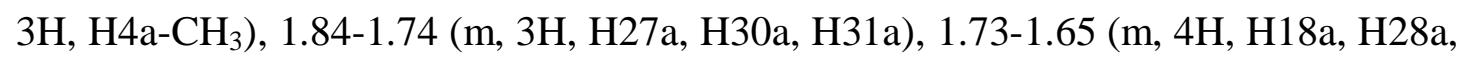
$\left.\mathrm{CH}_{2} \underline{\mathrm{CH}}_{2} \mathrm{CH}_{3}\right), 1.56$ (d, J=7.3 Hz, 3H, H26, H29), 1.49 (d, J= 7.1 Hz, 4H, H14a- $\mathrm{CH}_{3}$, H20b), 1.34-1.13 (m, 15H, H2'"b, H27b, H28b, H30b, H31b, H2'b, H5'-Me, H5"-Me, $\left.\mathrm{H} 12 \mathrm{a}-\mathrm{CH}_{3}\right), 1.02-0.80$ (m, 7H, H24a- $\left.\mathrm{CH}_{3}, \mathrm{H} 18 \mathrm{~b}, \mathrm{CH}_{2} \mathrm{CH}_{2} \underline{\mathrm{CH}}_{3}\right) .{ }^{13} \mathrm{C} \mathrm{NMR}(100 \mathrm{MHz}$, $\left.\mathrm{CDCl}_{3}\right) \delta 181.1,173.9,173.6,139.5,138.1,137.9,136.2,135.0,127.7,124.7,120.5$, 118.2, 118.1, 98.5, 95.7, 94.8, 81.8, 80.9, 80.4, 79.2, 79.1, 73.7, 68.4, 68.2, 68.2, 67.7, 67.0, 65.6, 57.1, 56.6, 55.5, 45.7, 40.3, 39.7, 38.9, 38.6, 36.7, 34.6, 34.3, 32.2, 31.4, 30.0, 29.6, 26.9, 26.6, 26.5, 25.5, 20.2, 19.9, 18.3, 17.9, 17.1, 16.6, 15.1, 13.5 .

S-36: The ${ }^{1} \mathrm{H}-\mathrm{NMR}$ and ${ }^{13} \mathrm{C}-\mathrm{NMR}$ data of $\mathbf{6 0}$

${ }^{1} \mathrm{H}$ NMR $\left(400 \mathrm{MHz}, \mathrm{CDCl}_{3}\right) \delta 10.89(\mathrm{~d}, J=9.9 \mathrm{~Hz}, 1 \mathrm{H}, \mathrm{CONHCS}), 8.92(\mathrm{~s}, 1 \mathrm{H}$, CSNH), 5.94-5.85 (m, 1H, H9), 5.83-5.66 (m, 3H, H10, H11, H23), 5.54 (dd, $J=9.9$, $2.5 \mathrm{~Hz}, 1 \mathrm{H}, \mathrm{H} 22), 5.47-5.33$ (m, 3H, H1", H3, H19), 5.01 (td, $J=10.6,9.9,5.6 \mathrm{~Hz}$, 2H, H4", H15), 4.77 (d, J = 3.8 Hz, 1H, H1) , 4.75-4.56 (m, 2H, H8a), 4.30 (s, 1H, H5), 4.16-4.09 (m, 1H, H5"), 4.05 (s, 1H, H7-OH), 3.97 (d, J=6.2 Hz, 1H, H6), 3.93 (s, 1H, H13), 3.90-3.73 (m, 3H, H17, H5', H3"'), 3.59 (ddd, J=11.2, 8.5, 4.7 Hz, 1H, H3'), 3.48 (s, 3H, H3"'-OMe), 3.40 (s, 3H, H3'-OMe), 3.33-3.18 (m, 3H, H2, H25, H4'), 2.52 (t, $J=7.7 \mathrm{~Hz}, 1 \mathrm{H}, \mathrm{H} 12), 2.38$ (d, $J=8.1 \mathrm{~Hz}, 1 \mathrm{H}, \mathrm{H} 5-\mathrm{OH}), 2.34-2.19$ (m, 4H, H16, H24, H2"a), 2.12-1.97 (m, 2H, H2'a, H20a), 1.87 (t, $J=2.0 \mathrm{~Hz}, 3 \mathrm{H}$,

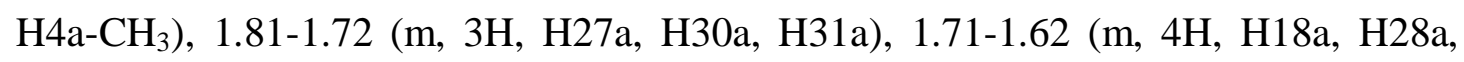
$\mathrm{C}_{3} \mathrm{H}_{5}$ ), 1.55 (s, 3H, H26, H29), 1.51-1.44 (m, 4H, H14a-CH 3 , H20b), 1.35-1.09 (m, 16H, H2'”b, H27b, H28b, H30b, H31b, H2'b, H5'-Me, H5"'-Me, H12a-CH $\mathrm{CH}_{3}, \mathrm{C}_{3}$ ), 0.94 (dd, $J=16.3,5.7 \mathrm{~Hz}, 5 \mathrm{H}, \mathrm{H} 24 \mathrm{a}-\mathrm{CH}_{3}, \mathrm{C}_{3} \mathrm{H}_{5}$ ), 0.85 (d, $\left.J=12.0 \mathrm{~Hz}, 1 \mathrm{H}, \mathrm{H} 18 \mathrm{~b}\right)$. ${ }^{13} \mathrm{C} \mathrm{NMR}\left(100 \mathrm{MHz}, \mathrm{CDCl}_{3}\right) \delta 180.8,174.8,173.6,139.5,138.0,137.8,136.2,135.0$, $127.7,124.7,120.5,118.2,118.1,98.5,95.7,94.8,81.8,81.0,80.4,79.2(2-\mathrm{C}), 77.3$, 73.7, 68.4, 68.2, 68.2, 67.7, 67.0, 65.7, 57.0, 56.6, 55.4, 45.7, 40.3, 39.7, 38.6, 36.6, $34.5,34.3,32.3,31.4,30.0,26.9,26.6,26.5,25.5,20.2,19.9,18.3,17.1,16.6,15.5$, 15.1, 9.7. 
S-37: The ${ }^{1} \mathrm{H}-\mathrm{NMR}$ and ${ }^{13} \mathrm{C}-\mathrm{NMR}$ data of $6 \mathrm{p}$ :

${ }^{1} \mathrm{H}$ NMR (400 MHz, $\mathrm{CDCl}_{3}$ ) $\delta 10.94$ (d, $\left.J=9.9 \mathrm{~Hz}, 1 \mathrm{H}, \mathrm{CONHCS}\right), 8.78$ (s, 1H, CSNH), 5.92-5.85 (m, 1H, H9), 5.81-5.65 (m, 3H, H10, H11, H23), 5.53 (dd, $J=9.9$, $2.5 \mathrm{~Hz}, 1 \mathrm{H}, \mathrm{H} 22), 5.49-5.34$ (m, 3H, H1", H3, H19), 5.00 (td, $J=10.8,10.3,3.7 \mathrm{~Hz}$, 2H, H4", H15), 4.77 (d, J = 3.9 Hz, 1H, H1'), 4.73-4.60 (m, 2H, H8a), 4.29 (d, J=6.3 Hz, 1H, H5), 4.17-4.04 (m, 2H, H5", H7-OH), 3.96 (d, J = 6.2 Hz, 1H, H6), 3.93 (s, 1H, H13), 3.89-3.71 (m, 3H, H17, H5', H3"), 3.60 (ddd, $J=11.2,8.6,4.8 \mathrm{~Hz}, 1 \mathrm{H}$, H3'), 3.48 (s, 3H, H3'--OMe), 3.40 (s, 3H, H3'-OMe), 3.33-3.19 (m, 3H, H2, H25, H4), 2.58-2.48 (m, 1H, H12), 2.42 (s, 1H, H5-OH), 2.34-2.15 (m, 6H, H16, H24, H2"'a, $\left.\mathrm{C}_{6} \mathrm{H}_{11}\right), 2.08\left(\mathrm{dd}, J=13.6,4.9 \mathrm{~Hz}, 1 \mathrm{H}, \mathrm{H}^{\prime} \mathrm{a}\right), 2.00(\mathrm{dd}, J=12.1,4.8 \mathrm{~Hz}, 1 \mathrm{H}$, H20a), 1.86 (s, 3H, H4a-CH $)$ 1.84-1.73 (m, 5H, H27a, H30a, H31a, $\mathrm{C}_{6} \mathrm{H}_{11}$ ), 1.71-1.64 (m, 2H, H18a, H28a), 1.55 (t, J=5.1 Hz, 3H, H26, H29), 1.52-1.39 (m, 6H, H14a- $\mathrm{CH}_{3}, \mathrm{H} 20 \mathrm{~b}, \mathrm{C}_{6} \mathrm{H}_{11}$ ), 1.35-1.09 (m, 20H, H2'b, H27b, H28b, H30b, H31b, H2 b, H5'-Me, H5"-Me, H12a-CH ${ }_{3}, \mathrm{C}_{6} \mathrm{H}_{11}$ ), 0.92 (d, $J=7.1 \mathrm{~Hz}, 3 \mathrm{H}, \mathrm{H} 24 \mathrm{a}-\mathrm{CH}_{3}$ ), 0.87-0.81 (m, 1H, H18b). ${ }^{13} \mathrm{C}$ NMR (100 MHz, $\left.\mathrm{CDCl}_{3}\right) \delta 181.3,176.8,173.6,139.5,138.1$, 137.9, 136.2, 135.0, 127.7, 124.7, 120.5, 118.2, 118.1, 98.5, 95.7, 94.8, 81.8, 80.9, 80.4, 79.2, 79.1, 77.2, 73.8, 68.4, 68.2, 68.1, 67.7, 67.0, 65.7, 57.1, 56.6, 55.5, 45.8, 45.7, 40.3, 39.7, 38.6, 36.7, 34.6, 34.3, 32.3, 31.4, 30.0, 29.0, 28.9, 26.9, 26.6, 26.5, 25.5, 25.4, 25.3, 25.3, 20.2, 20.0, 18.3, 17.1, 16.6, 15.1 .

S-38: The ${ }^{1} \mathrm{H}-\mathrm{NMR}$ and ${ }^{13} \mathrm{C}-\mathrm{NMR}$ data of $\mathbf{6 q}$ :

${ }^{1} \mathrm{H}$ NMR (400 MHz, $\left.\mathrm{CDCl}_{3}\right) \delta 10.92(\mathrm{~d}, J=9.9 \mathrm{~Hz}, 1 \mathrm{H}, \mathrm{CONHCS}), 9.13$ (d, $J=$ $8.4 \mathrm{~Hz}, 1 \mathrm{H}, \mathrm{CSNH}), 5.87$ (d, $J=9.8 \mathrm{~Hz}, 1 \mathrm{H}, \mathrm{H} 9), 5.80-5.65$ (m, 3H, H10, H11, H23), 5.52 (dd, $J=9.9,2.4 \mathrm{~Hz}, 1 \mathrm{H}, \mathrm{H} 22), 5.47-5.30$ (m, 3H, H1" , H3, H19), 4.99 (td, $J=$ 9.6, 3.5 Hz, 2H, H4", H15), 4.76 (d, $J=3.9 \mathrm{~Hz}, 1 \mathrm{H}, \mathrm{H} 1$ '), 4.68 (t, $J=10.1 \mathrm{~Hz}, 2 \mathrm{H}$, H8a), 4.29 (d, $J=6.2 \mathrm{~Hz}, 1 \mathrm{H}, \mathrm{H} 5), 4.14-4.05$ (m, 2H, H5", H7-OH), 3.95 (dd, $J=6.3$, $1.9 \mathrm{~Hz}, 1 \mathrm{H}, \mathrm{H6}), 3.92$ (s, 1H, H13), 3.88-3.70 (m, 3H, H17, H5', H3"), 3.59 (td, $J=$ 11.6, 10.2, $4.9 \mathrm{~Hz}, 1 \mathrm{H}, \mathrm{H} 3$ ), 3.46 (s, 3H, H3"-OMe), 3.40 (s, 3H, H3'-OMe), 3.32-3.17 (m, 3H, H2, H25, H4), 2.51 (t, $J=8.1 \mathrm{~Hz}, 1 \mathrm{H}, \mathrm{H} 12), 2.37-2.15$ (m, 7H, H5-OH, H16, H24, H2"a, $\underline{\mathrm{CH}}_{2} \mathrm{CH}_{2} \mathrm{CH}_{2} \mathrm{CH}_{2} \mathrm{CH}_{3}$ ), 2.07 (dd, $J=13.8,5.0 \mathrm{~Hz}, 1 \mathrm{H}$, $\left.\mathrm{H}_{2}^{\prime} \mathrm{a}\right), 2.03-1.95$ (m, 1H, H20a), 1.85 (s, 3H, H4a-CH $), 1.81-1.72$ (m, 3H, H27a, 
H30a, H31a), 1.70-1.63 (m, 2H, H18a, H28a), 1.54 (d, J = 7.6 Hz, 3H, H26, H29), 1.44 (d, $\left.J=29.1 \mathrm{~Hz}, 4 \mathrm{H}, \mathrm{H} 14 \mathrm{a}-\mathrm{CH}_{3}, \mathrm{H} 20 \mathrm{~b}\right), 1.36-1.10$ (m, 19H, H2'”b, H27b, H28b, $\mathrm{H} 30 \mathrm{~b}, \mathrm{H} 31 \mathrm{~b}, \mathrm{H} 2$ 'b, H5'-Me, H5"'-Me, H12a- $\left.\mathrm{CH}_{3}, \mathrm{CH}_{2} \underline{\mathrm{CH}}_{2}{ }_{2} \mathrm{CH}_{2} \mathrm{CH}_{2} \mathrm{CH}_{3}\right), 0.93-0.85$ (m, 8H, H24a- $\mathrm{CH}_{3}, \mathrm{CH}_{2} \mathrm{CH}_{2} \mathrm{CH}_{2} \mathrm{CH}_{2} \underline{\mathrm{CH}_{3}}$ ), 0.83-0.71 (m, 1H, H18b). ${ }^{13} \mathrm{C}$ NMR (100 $\left.\mathrm{MHz}, \mathrm{CDCl}_{3}\right) \delta 181.1,174.2,173.6,139.4,138.0,137.8,136.1,135.0,127.7,124.7$, $120.5,118.2,118.1,98.5,95.7,94.8,81.8,80.9,80.3,79.2,73.7,68.4,68.3,68.2$, 67.7, 67.0, 65.6, 57.1, 56.6, 55.4, 45.7, 40.3, 39.7, 38.6, 37.0, 36.6, 34.5, 34.3, 32.2, $31.4,31.2,31.1,30.0,26.9,26.6,26.5,25.8,25.5,24.1,22.3,20.2,19.9$, 18.3, 17.1, $16.6,15.1,13.8$.

S-39: The ${ }^{1} \mathrm{H}-\mathrm{NMR}$ and ${ }^{13} \mathrm{C}-\mathrm{NMR}$ data of 6r:

${ }^{1} \mathrm{H}$ NMR (400 MHz, $\left.\mathrm{CDCl}_{3}\right) \delta 10.98(\mathrm{~d}, J=9.9 \mathrm{~Hz}, 1 \mathrm{H}, \mathrm{CONHCS}), 9.50$ (d, $J=$ $14.7 \mathrm{~Hz}, 1 \mathrm{H}, \mathrm{CSNH}), 8.11$ (td, $J=8.9,6.3 \mathrm{~Hz}, 1 \mathrm{H}, \mathrm{Ph}), 7.06$ (ddd, $J=9.2,7.5,2.4 \mathrm{~Hz}$, 1H, Ph), 6.95 (ddd, $J=11.1,8.3,2.4 \mathrm{~Hz}, 1 \mathrm{H}, \mathrm{Ph}$ ), 5.91-5.85 (m, 1H, H9), 5.83-5.66 (m, 3H, H10, H11, H23), 5.59-5.48 (m, 2H, H22, H1"), 5.46-5.34 (m, 2H, H3, H19), $5.09\left(\mathrm{dd}, J=10.1,3.6 \mathrm{~Hz}, 1 \mathrm{H}, \mathrm{H} 4^{\prime \prime}\right), 5.00$ (d, $\left.J=10.5 \mathrm{~Hz}, 1 \mathrm{H}, \mathrm{H} 15\right), 4.79$ (d, $J=3.9$ Hz, 1H, H1'), 4.75-4.62 (m, 2H, H8a), 4.29 (d, $J=6.1 \mathrm{~Hz}, 1 \mathrm{H}, \mathrm{H} 5), 4.18$ (q, $J=6.5$ Hz, 1H, H5"), 4.05 (s, 1H, H7-OH), 3.97 (d, J = 6.3 Hz, 1H, H6), 3.94 (s, 1H, H13), 3.91-3.75 (m, 3H, H17, H5', H3'"), 3.62 (ddd, $J=11.4,8.6,4.8$ Hz, 1H, H3'), 3.50 (s, 3H, H3"'-OMe), 3.43 (s, 3H, H3'-OMe), 3.33-3.20 (m, 3H, H2, H25, H4'), 2.57-2.49 (m, 1H, H12), 2.40 (s, 1H, H5-OH), 2.35-2.20 (m, 4H, H16, H24, H2" "a), 2.13 (dd, J $\left.=13.5,4.9 \mathrm{~Hz}, 1 \mathrm{H}, \mathrm{H}^{\prime} \mathrm{a}\right), 2.00(\mathrm{dd}, J=9.7,3.2 \mathrm{~Hz}, 1 \mathrm{H}, \mathrm{H} 20 \mathrm{a}), 1.87$ (t, $J=2.0 \mathrm{~Hz}$, 3H, H4a-CH $)$, 1.84-1.73 (m, 3H, H27a, H30a, H31a), 1.74-1.65 (m, 2H, H18a, H28a), 1.55 (d, $J=7.9 \mathrm{~Hz}, 3 \mathrm{H}, \mathrm{H} 26, \mathrm{H} 29), 1.48$ (d, $J=8.0 \mathrm{~Hz}, 4 \mathrm{H}, \mathrm{H} 14 \mathrm{a}-\mathrm{CH}_{3}, \mathrm{H} 20 \mathrm{~b}$ ), 1.34-1.13 (m, 15H, H2'b, H27b, H28b, H30b, H31b, H2'b, H5'-Me, H5"-Me, $\left.\mathrm{H} 12 \mathrm{a}-\mathrm{CH}_{3}\right), 0.92$ (d, $\left.J=7.1 \mathrm{~Hz}, 3 \mathrm{H}, \mathrm{H} 24 \mathrm{a}-\mathrm{CH}_{3}\right), 0.85$ (d, $\left.J=12.4 \mathrm{~Hz}, 1 \mathrm{H}, \mathrm{H} 18 \mathrm{~b}\right) .{ }^{13} \mathrm{C}$ NMR (100 MHz, $\left.\mathrm{CDCl}_{3}\right) \delta 180.9,173.6,164.8,161.8,159.7,139.5,138.1,137.9$, 136.2, 135.0, 134.2, 127.7, 124.7, 120.5, 118.2, 118.0, 113.3, 113.1, 98.7, 95.7, 94.8, 81.8, 81.1, 80.4, 79.2, 79.1, 73.7, 68.4, 68.2, 68.2, 67.7, 67.0, 65.7, 60.4, 57.2, 56.6, 55.8, 45.7, 40.3, 39.7, 38.7, 36.7, 34.6, 34.3, 32.4, 31.4, 30.0, 27.0, 26.6, 26.5, 25.5, $20.2,19.9,18.3,17.1,16.6,15.1,14.2$. 
S-40: The ${ }^{1} \mathrm{H}-\mathrm{NMR}$ and ${ }^{13} \mathrm{C}-\mathrm{NMR}$ data of 6s:

${ }^{1} \mathrm{H}$ NMR $\left(400 \mathrm{MHz}, \mathrm{CDCl}_{3}\right) \delta 10.93(\mathrm{~d}, J=9.8 \mathrm{~Hz}, 1 \mathrm{H}, \mathrm{CONHCS}), 9.58(\mathrm{~d}, J=$ 15.4 Hz, 1H, CSNH), 7.77 (ddd, $J=8.8,5.8,3.3 \mathrm{~Hz}, 1 \mathrm{H}, \mathrm{Ph}), 7.33-7.27$ (m, 1H, Ph), 7.20 (ddd, $J=10.8,9.0,4.1 \mathrm{~Hz}, 1 \mathrm{H}, \mathrm{Ph}), 5.92-5.86$ (m, 1H, H9), 5.82-5.67 (m, 3H, H10, H11, H23), 5.59-5.48 (m, 2H, H22, H1'), 5.42 (br s, 2H, H3, H19), 5.09 (dd, J = 10.1, 3.5 Hz, 1H, H4" ), 5.00 (d, $J=10.6 \mathrm{~Hz}, 1 \mathrm{H}, \mathrm{H} 15), 4.79$ (d, $J=3.8 \mathrm{~Hz}, 1 \mathrm{H}, \mathrm{H} 1$ '), 4.74-4.63 (m, 2H, H8a), 4.29 (d, $J=6.6 \mathrm{~Hz}, 1 \mathrm{H}, \mathrm{H} 5), 4.18$ (q, $\left.J=6.4 \mathrm{~Hz}, 1 \mathrm{H}, \mathrm{H} 5{ }^{\prime \prime}\right)$, 4.05 (s, 1H, H7-OH), 3.97 (d, J = 6.2 Hz, 1H, H6), 3.94 (s, 1H, H13), 3.91-3.76 (m, 3H, H17, H5', H3"), 3.62 (ddd, $J=11.3,8.5,4.8 \mathrm{~Hz}, 1 \mathrm{H}, \mathrm{H} 3$ ), 3.51 (s, 3H, H3"'-OMe), 3.44 (s, 3H, H3'-OMe), 3.34-3.20 (m, 3H, H2, H25, H4'), 2.57-2.49 (m, 1H, H12), 2.38 (d, J = 8.8 Hz, 1H, H5-OH), 2.34-2.20 (m, 4H, H16, H24, H2"'a), 2.14 (dd, $\left.J=13.6,4.9 \mathrm{~Hz}, 1 \mathrm{H}, \mathrm{H}_{2}^{\prime} \mathrm{a}\right), 2.02-1.96$ (m, 1H, H20a), 1.87 (t, $J=2.0 \mathrm{~Hz}, 3 \mathrm{H}$,

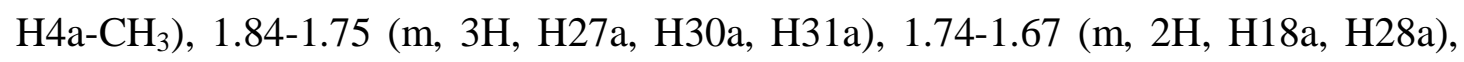
1.56 (d, $J=8.2 \mathrm{~Hz}, 3 \mathrm{H}, \mathrm{H} 26, \mathrm{H} 29$ ), 1.49 (d, $J=7.8 \mathrm{~Hz}, 4 \mathrm{H}, \mathrm{H} 14 \mathrm{a}-\mathrm{CH}_{3}, \mathrm{H} 20 \mathrm{~b}$ ), 1.34-1.13 (m, 15H, H2'b, H27b, H28b, H30b, H31b, H2'b, H5'-Me, H5"-Me, $\left.\mathrm{H} 12 \mathrm{a}-\mathrm{CH}_{3}\right), 0.92$ (d, $\left.J=7.1 \mathrm{~Hz}, 3 \mathrm{H}, \mathrm{H} 24 \mathrm{a}-\mathrm{CH}_{3}\right), 0.85$ (d, $\left.J=12.5 \mathrm{~Hz}, 1 \mathrm{H}, \mathrm{H} 18 \mathrm{~b}\right) .{ }^{13} \mathrm{C}$ NMR $\left(100 \mathrm{MHz}, \mathrm{CDCl}_{3}\right) \delta 180.7,173.6,161.5,157.7,155.2,139.5,138.1,137.9$, 136.2, 135.0, 127.7, 124.7, 122.5, 122.3, 120.5, 118.4, 118.2, 118.0, 98.7, 95.7, 94.8, 81.8, 81.1, 80.4, 79.2, 79.1, 73.7, 68.4, 68.2, 68.2, 67.7, 67.0, 65.7, 60.4, 57.2, 56.6, 55.8, 45.7, 40.3, 39.7, 38.7, 36.7, 34.6, 34.3, 32.4, 31.4, 30.0, 27.0, 26.6, 26.5, 25.5, $20.2,20.0,18.3,17.2,16.6,15.2,14.2$.

S-41: The ${ }^{1} \mathrm{H}-\mathrm{NMR}$ and ${ }^{13} \mathrm{C}-\mathrm{NMR}$ data of $\mathbf{6 t}$ :

${ }^{1} \mathrm{H}$ NMR $\left(400 \mathrm{MHz}, \mathrm{CDCl}_{3}\right) \delta 10.73(\mathrm{~d}, J=9.8 \mathrm{~Hz}, 1 \mathrm{H}, \mathrm{CONHCS}), 9.07$ (s, 1H, CSNH), 7.49 (tt, $J=8.3,6.1 \mathrm{~Hz}, 1 \mathrm{H}, \mathrm{Ph}), 7.02(\mathrm{t}, J=8.7 \mathrm{~Hz}, 2 \mathrm{H}, \mathrm{Ph}), 5.92-5.84(\mathrm{~m}$, 1H, H9), 5.83-5.67 (m, 3H, H10, H11, H23), 5.57-5.47 (m, 2H, H22, H1 "), 5.46-5.32 (m, $J=4.7 \mathrm{~Hz}, 2 \mathrm{H}, \mathrm{H} 3, \mathrm{H} 19), 5.08$ (dd, $\left.J=10.0,3.5 \mathrm{~Hz}, 1 \mathrm{H}, \mathrm{H} 4{ }^{\prime \prime}\right), 5.03-4.96$ (m, 1H, H15), 4.78 (d, J=3.8 Hz, 1H, H1'), 4.73-4.60 (m, 2H, H8a), 4.29 (d, $J=6.3 \mathrm{~Hz}, 1 \mathrm{H}$, H5), 4.16 (q, $\left.J=6.6 \mathrm{~Hz}, 1 \mathrm{H}, \mathrm{H} 5^{\prime \prime}\right), 3.96$ (d, $\left.J=6.3 \mathrm{~Hz}, 1 \mathrm{H}, \mathrm{H} 6\right), 3.94$ (s, 1H, H13), 3.91-3.76 (m, 3H, H17, H5', H3"), 3.67-3.57 (m, 1H, H3'), 3.50 (s, 3H, H3"'-OMe), 3.42 (s, 3H, H3'-OMe), 3.33-3.18 (m, 3H, H2, H25, H4), 2.52 (t, J = 8.2 Hz, 1H, 
H12), 2.38-2.18 (m, 5H, H5-OH, H16, H24, H2"a), 2.12 (dd, $J=13.7,5.0 \mathrm{~Hz}, 1 \mathrm{H}$, H2'a), 1.99 (dd, $J=12.1,4.8 \mathrm{~Hz}, 1 \mathrm{H}, \mathrm{H} 20 \mathrm{a}), 1.86$ (t, $\left.J=2.0 \mathrm{~Hz}, 3 \mathrm{H}, \mathrm{H} 4 \mathrm{a}-\mathrm{CH}_{3}\right)$, 1.83-1.71 (m, 3H, H27a, H30a, H31a), 1.72-1.61 (m, 2H, H18a, H28a), 1.55 (d, $J=$ $8.0 \mathrm{~Hz}, 3 \mathrm{H}, \mathrm{H} 26, \mathrm{H} 29), 1.48$ (d, $J=10.4 \mathrm{~Hz}, 4 \mathrm{H}, \mathrm{H} 14 \mathrm{a}-\mathrm{CH}_{3}, \mathrm{H} 20 \mathrm{~b}$ ), 1.33-1.13 (m, 15H, H2'b, H27b, H28b, H30b, H31b, H2'b, H5'-Me, H5"-Me, H12a-CH3), 0.92 (d, $\left.J=7.1 \mathrm{~Hz}, 3 \mathrm{H}, \mathrm{H} 24 \mathrm{a}-\mathrm{CH}_{3}\right), 0.88-0.82(\mathrm{~m}, 1 \mathrm{H}, \mathrm{H} 18 \mathrm{~b}) .{ }^{13} \mathrm{C} \mathrm{NMR}\left(100 \mathrm{MHz}, \mathrm{CDCl}_{3}\right) \delta$ $180.6,173.6,161.5,161.4,160.1,158.9,158.9,139.5,138.1,137.9,136.2,135.0$, $127.7,124.7,120.5,118.2,118.1,112.7,112.5,112.4,98.5,95.7,94.8,81.8,80.9$, 80.4, 79.2, 79.1, 73.8, 68.4, 68.3, 68.2, 67.7, 67.0, 65.7, 57.2, 56.6, 55.8, 45.7, 40.3, $39.7,38.6,36.7,34.6,34.3,32.3,31.4,30.0,26.9,26.6,26.5,25.5,20.2,19.9,18.3$, $17.2,16.6,15.1$.

S-42: The ${ }^{1} \mathrm{H}-\mathrm{NMR}$ and ${ }^{13} \mathrm{C}-\mathrm{NMR}$ data of $6 \mathrm{u}$ :

${ }^{1} \mathrm{H}$ NMR (400 MHz, $\mathrm{CDCl}_{3}$ ) $\delta 11.07$ (d, $\left.J=9.8 \mathrm{~Hz}, 1 \mathrm{H}, \mathrm{CONHCS}\right), 8.97(\mathrm{~s}, 1 \mathrm{H}$, CSNH), 6.94 (d, $J=2.3 \mathrm{~Hz}, 2 \mathrm{H}, \mathrm{Ph}), 6.67$ (d, $J=2.3 \mathrm{~Hz}, 1 \mathrm{H}, \mathrm{Ph}), 5.89$ (d, $J=10.0$ Hz, 1H, H9), 5.82-5.66 (m, 3H, H10, H11, H23), 5.59-5.48 (m, 2H, H22, H1'), 5.43 (br s, 2H, H3, H19), 5.08 (d, $J=9.5 \mathrm{~Hz}, 1 \mathrm{H}, \mathrm{H} 4^{\prime \prime}$ ), 5.00 (d, $J=10.7 \mathrm{~Hz}, 1 \mathrm{H}, \mathrm{H} 15$ ), $\left.4.79(\mathrm{~d}, J=3.8 \mathrm{~Hz}, 1 \mathrm{H}, \mathrm{H1})^{\prime}\right), 4.69$ (t, $\left.J=3.1 \mathrm{~Hz}, 2 \mathrm{H}, \mathrm{H} 8 \mathrm{a}\right), 4.30$ (t, $J=6.9 \mathrm{~Hz}, 1 \mathrm{H}$, H5), 4.18 (d, $\left.J=6.6 \mathrm{~Hz}, 1 \mathrm{H}, \mathrm{H} 5^{\prime \prime}\right), 4.05$ (s, 1H, H7-OH), 3.98 (d, J=6.2 Hz, 1H, H6), 3.94 (s, 1H, H13), 3.91-3.77 (m, 3H, H17, H5', H3') , 3.83 (s, 6H, 20CH3), 3.67-3.58 (m, 1H, H3'), 3.52 (s, 3H, H3"'-OMe), 3.43 (s, 3H, H3'-OMe), 3.34-3.22 (m, 3H, H2, H25, H4'), 2.53 (t, $J=7.8 \mathrm{~Hz}, 1 \mathrm{H}, \mathrm{H} 12), 2.38$ (d, $J=8.1 \mathrm{~Hz}, 1 \mathrm{H}, \mathrm{H} 5-\mathrm{OH}), 2.34-2.21$ (m, 4H, H16, H24, H2"a), 2.14 (dd, $\left.J=13.6,4.9 \mathrm{~Hz}, 1 \mathrm{H}, \mathrm{H} 2^{\prime} \mathrm{a}\right), 2.00$ (dd, $J=12.2$, $4.8 \mathrm{~Hz}, 1 \mathrm{H}, \mathrm{H} 20 \mathrm{a}), 1.87$ (t, J = 2.0 Hz, 3H, H4a-CH $), 1.83-1.74$ (m, 3H, H27a, H30a, H31a), 1.73-1.65 (m, 2H, H18a, H28a), 1.56 (d, J = 8.6 Hz, 3H, H26, H29), 1.49 (d, $J$ $\left.=6.8 \mathrm{~Hz}, 4 \mathrm{H}, \mathrm{H} 14 \mathrm{a}-\mathrm{CH}_{3}, \mathrm{H} 20 \mathrm{~b}\right), 1.36-1.14$ (m, 15H, H2' b, H27b, H28b, H30b, H31b, H2'b, H5'-Me, H5"-Me, H12a-CH ${ }_{3}$ ), 0.93 (d, $\left.J=7.1 \mathrm{~Hz}, 3 \mathrm{H}, \mathrm{H} 24 \mathrm{a}-\mathrm{CH}_{3}\right), 0.86$ (d, $J=$ $12.6 \mathrm{~Hz}, 1 \mathrm{H}, \mathrm{H} 18 \mathrm{~b}) .{ }^{13} \mathrm{C} \mathrm{NMR}\left(100 \mathrm{MHz}, \mathrm{CDCl}_{3}\right) \delta 181.1,173.6,166.8,161.1(2-\mathrm{C})$, $139.5,138.1,137.9,136.2$, 135.0, 133.9, 127.7, 124.7, 120.5, 118.2, 118.1, 105.7, 105.2(2-C), 98.6, 95.7, 94.8, 81.8, 81.0, 80.4, 79.2, 79.1,77.2, 73.8, 68.4, 68.2, 68.2, $67.7,67.0,65.7,57.2,56.6,55.8,55.7(2-\mathrm{C}), 45.7,40.3,39.7,38.6,36.7,34.6,34.3$, 
$32.3,31.4,30.0,27.0,26.6,26.5,25.5,20.2,19.9,18.3,17.2,16.6,15.1$.

S-43: The ${ }^{1} \mathrm{H}-\mathrm{NMR}$ and ${ }^{13} \mathrm{C}-\mathrm{NMR}$ data of $6 \mathbf{v}$ :

${ }^{1} \mathrm{H}$ NMR (400 MHz, $\left.\mathrm{CDCl}_{3}\right) \delta 11.15$ (d, $\left.J=9.8 \mathrm{~Hz}, 1 \mathrm{H}, \mathrm{CONHCS}\right), 9.24$ (s, 1H, CSNH), 7.06 (s, 2H, Ph), 5.90-5.81 (m, 1H, H9), 5.80-5.64 (m, 3H, H10, H11, H23), 5.56-5.46 (m, 2H, H22, H1"), 5.39 (s, 2H, H3, H19), 5.05 (d, J = 10.0 Hz, 1H, H4"'), 5.02-4.94 (m, 1H, H15), 4.82-4.73 (m, 1H, H1'), 4.70-4.57 (m, 2H, H8a), 4.27 (d, J = $6.2 \mathrm{~Hz}, 1 \mathrm{H}, \mathrm{H} 5), 4.16$ (d, J = 6.8 Hz, 1H, H5"), 4.11-4.03 (s, 1H, H7-OH), 3.97-3.74 (m, 14H, H6, H13, H17, H5', H3", 30CH $\left.{ }_{3}\right), 3.65-3.55$ (m, 1H, H3) $) 3.49$ (s, 3H, H3"'-OMe), 3.40 (s, 3H, H3'-OMe), 3.32-3.18 (m, 3H, H2, H25, H4'), 2.61-2.43 (m, 2H, H12, H5-OH), 2.34-2.18 (m, 4H, H16, H24, H2"a), 2.11 (dd, $J=14.0,4.7 \mathrm{~Hz}$, 1H, H2' a), 2.02-1.94 (m, 1H, H20a), 1.83 (s, 3H, H4a-CH $), 1.80-1.73$ (m, 3H, H27a, H30a, H31a), 1.69-1.59 (m, 2H, H18a, H28a), 1.53 (d, $J=9.0 \mathrm{~Hz}, 3 \mathrm{H}, \mathrm{H} 26, \mathrm{H} 29$ ), 1.47 (s, 4H, H14a-CH, H20b), 1.34-1.01 (m, 15H, H2'b, H27b, H28b, H30b, H31b, H2'b, H5'-Me, H5"'-Me, H12a-CH $\left.{ }_{3}\right), 0.90$ (d, $\left.J=7.5 \mathrm{~Hz}, 3 \mathrm{H}, \mathrm{H} 24 \mathrm{a}-\mathrm{CH}_{3}\right), 0.83$ (d, $J=$ $11.9 \mathrm{~Hz}, 1 \mathrm{H}, \mathrm{H} 18 \mathrm{~b}) .{ }^{13} \mathrm{C} \mathrm{NMR}\left(100 \mathrm{MHz}, \mathrm{CDCl}_{3}\right) \delta 181.2,173.6,166.8,153.3(2-\mathrm{C})$, $142.5,139.5,138.0,137.8,136.1,135.0,127.7,126.8,124.7,120.4,118.2,118.0$, 105.0(2-C), 98.6, 95.7, 94.8, 81.8, 81.1, 80.4, 79.2, 79.2, 77.4, 73.8, 68.3, 68.2, 68.1, 67.7, 67.0, 65.7, 61.0, 57.1, 56.6, 56.4(2-C), 55.7, 45.7, 40.3, 39.7, 38.6, 36.6, 34.6, $34.3,32.3,31.4,30.0,26.9,26.6,26.4,25.5,20.2,19.9,18.3,17.2,16.6,15.1$. 
S-44: The ${ }^{1} \mathrm{H}-\mathrm{NMR}$ and ${ }^{13} \mathrm{C}-\mathrm{NMR}$ spectrums of $\mathbf{5 a}$
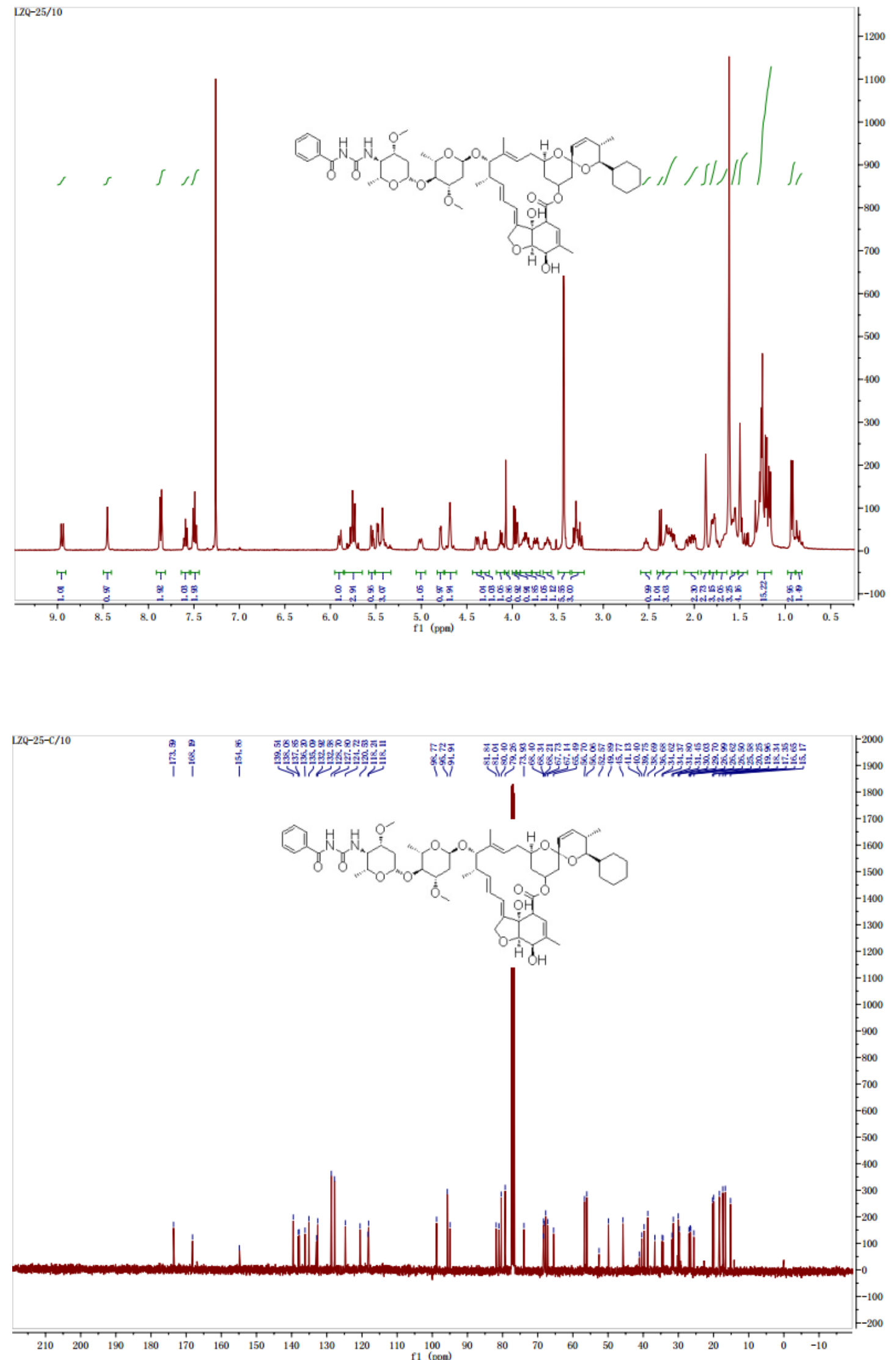
S-45: The ${ }^{1} \mathrm{H}-\mathrm{NMR}$ and ${ }^{13} \mathrm{C}-\mathrm{NMR}$ spectrums of $\mathbf{5 b}$
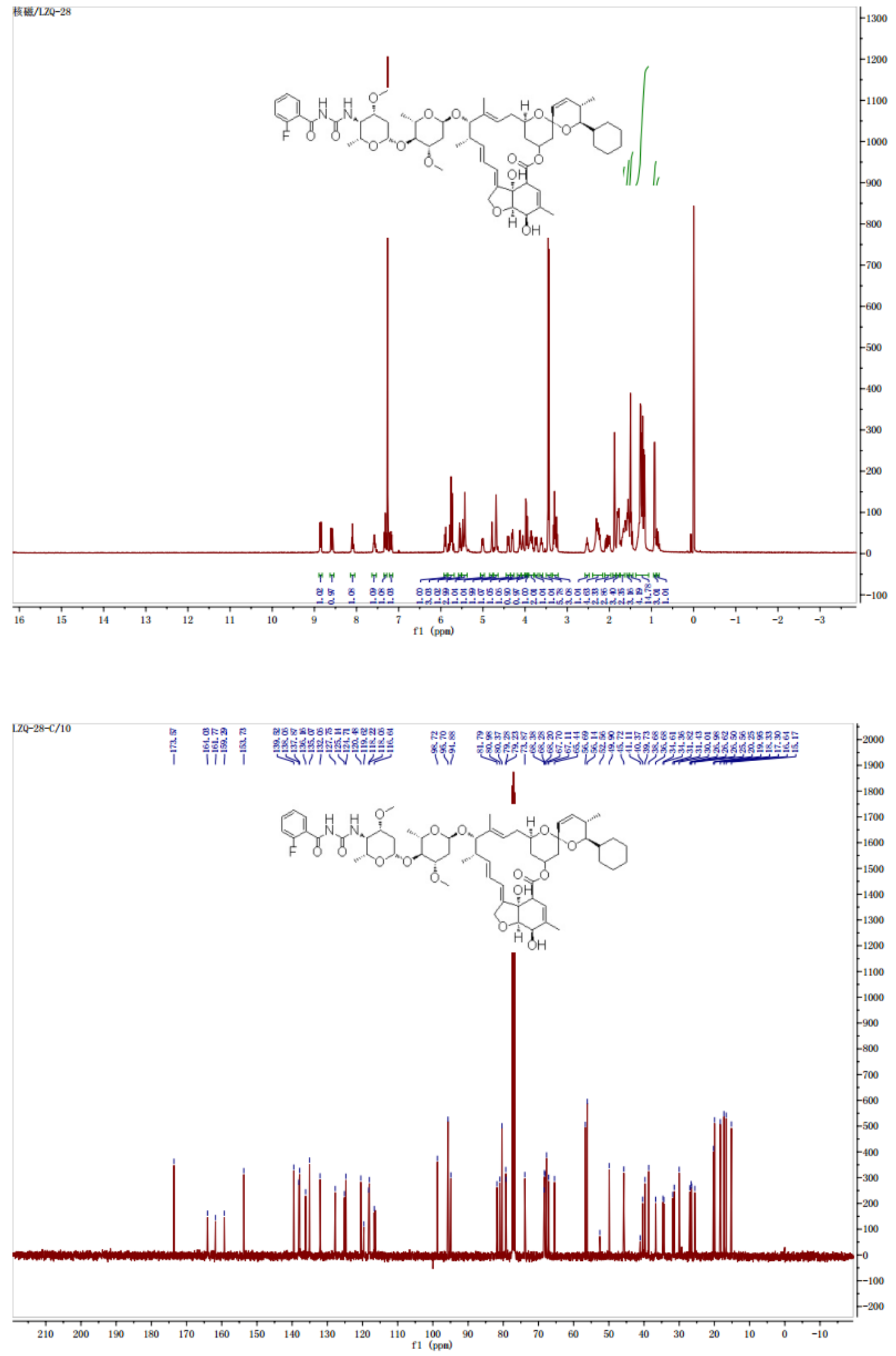
S-46: The ${ }^{1} \mathrm{H}-\mathrm{NMR}$ and ${ }^{13} \mathrm{C}-\mathrm{NMR}$ spectrums of $\mathbf{5 c}$
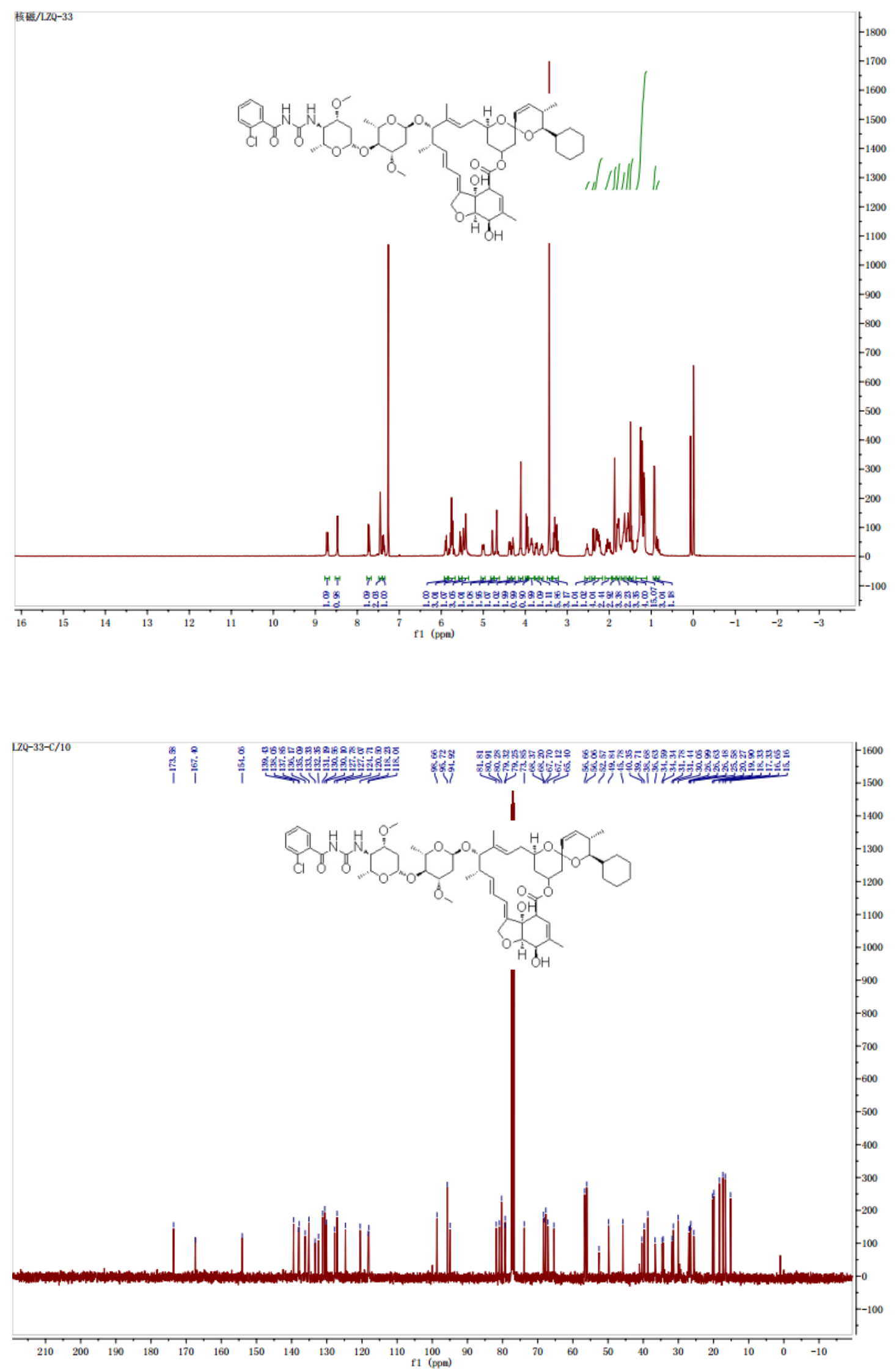
S-47: The ${ }^{1} \mathrm{H}-\mathrm{NMR}$ and ${ }^{13} \mathrm{C}-\mathrm{NMR}$ spectrums of $\mathbf{5 d}$
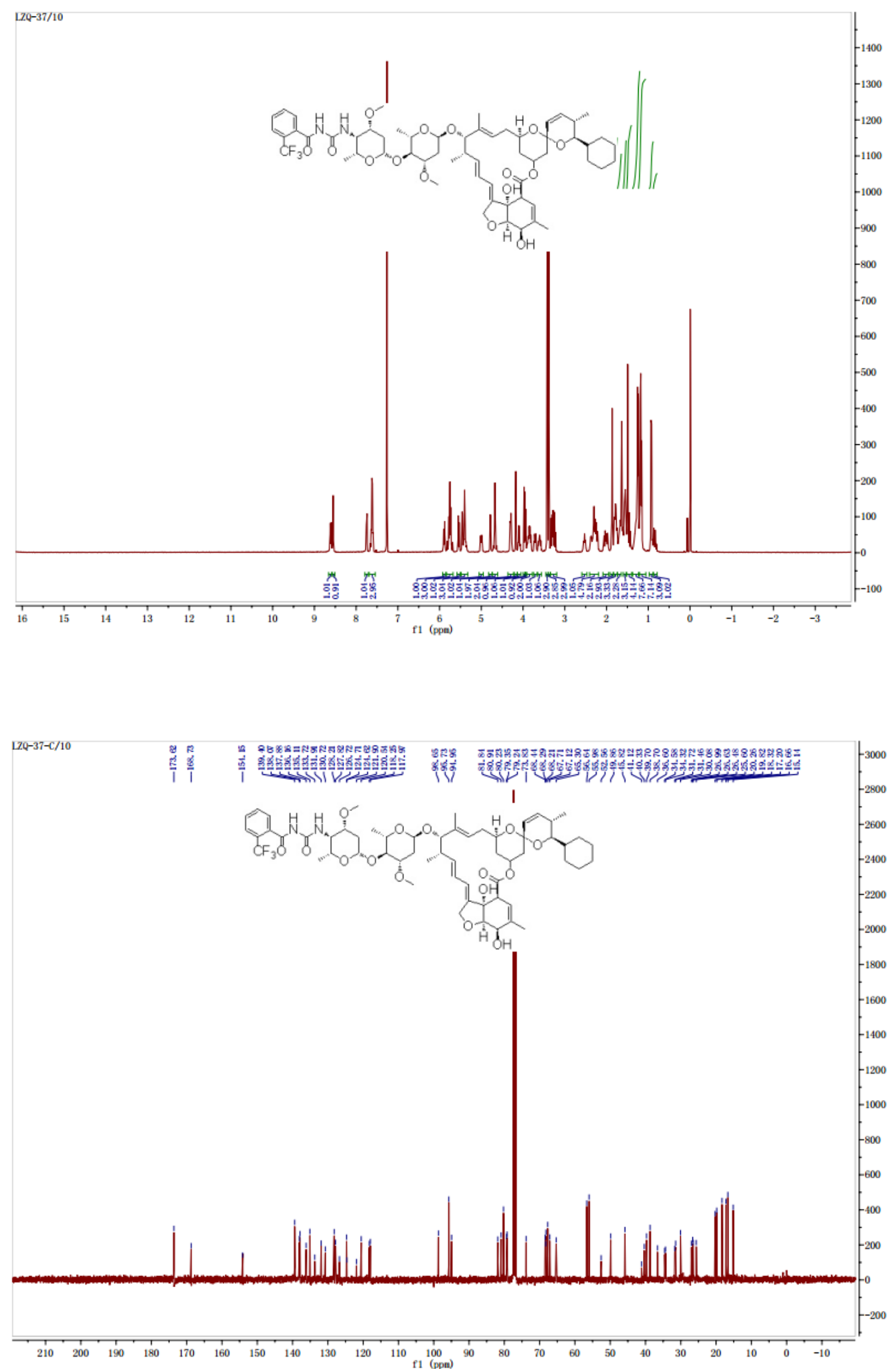
S-48: The ${ }^{1} \mathrm{H}-\mathrm{NMR}$ and ${ }^{13} \mathrm{C}-\mathrm{NMR}$ spectrums of $\mathbf{5 e}$
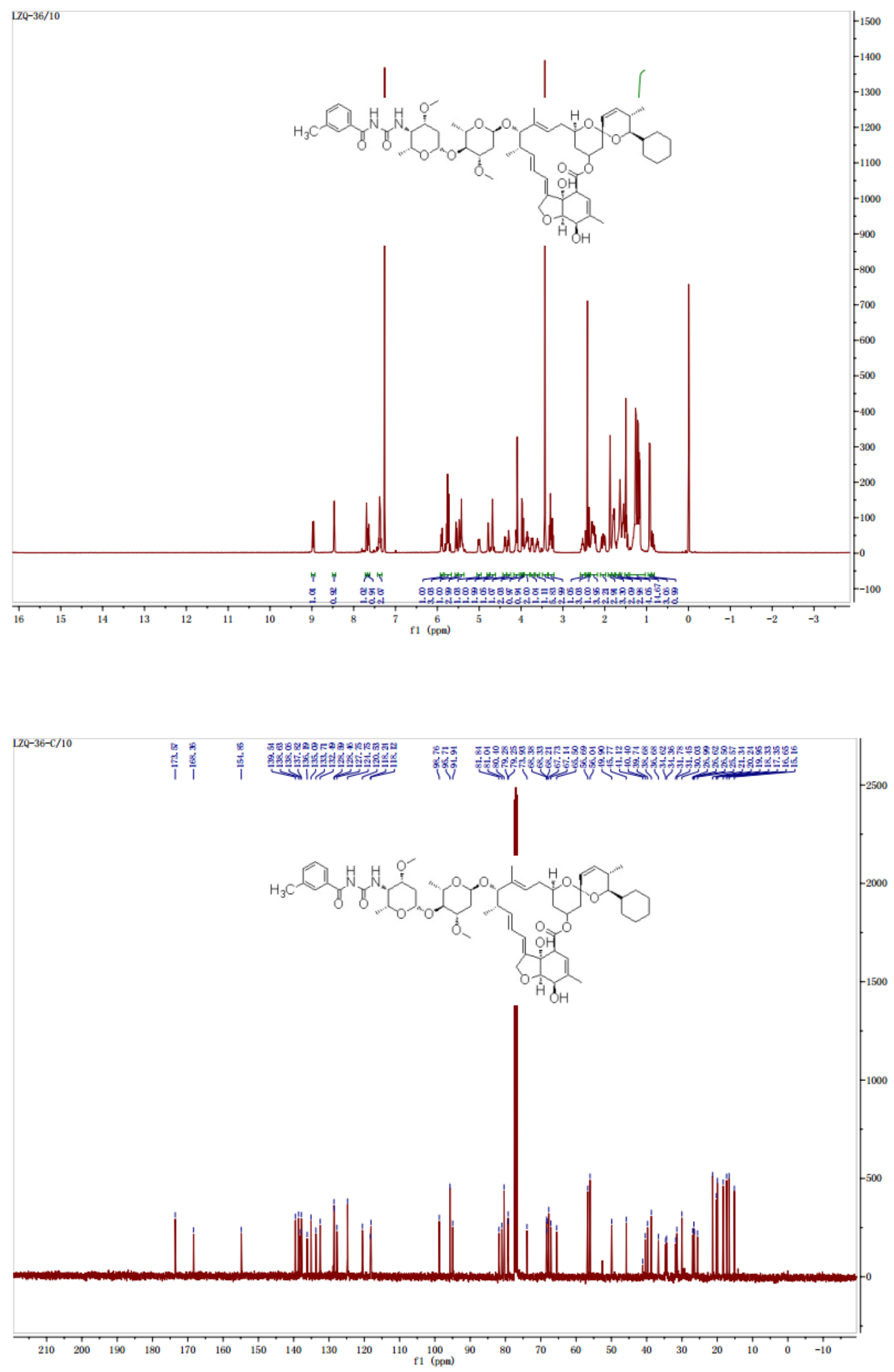
S-49: The ${ }^{1} \mathrm{H}-\mathrm{NMR}$ and ${ }^{13} \mathrm{C}-\mathrm{NMR}$ spectrums of $\mathbf{5 f}$
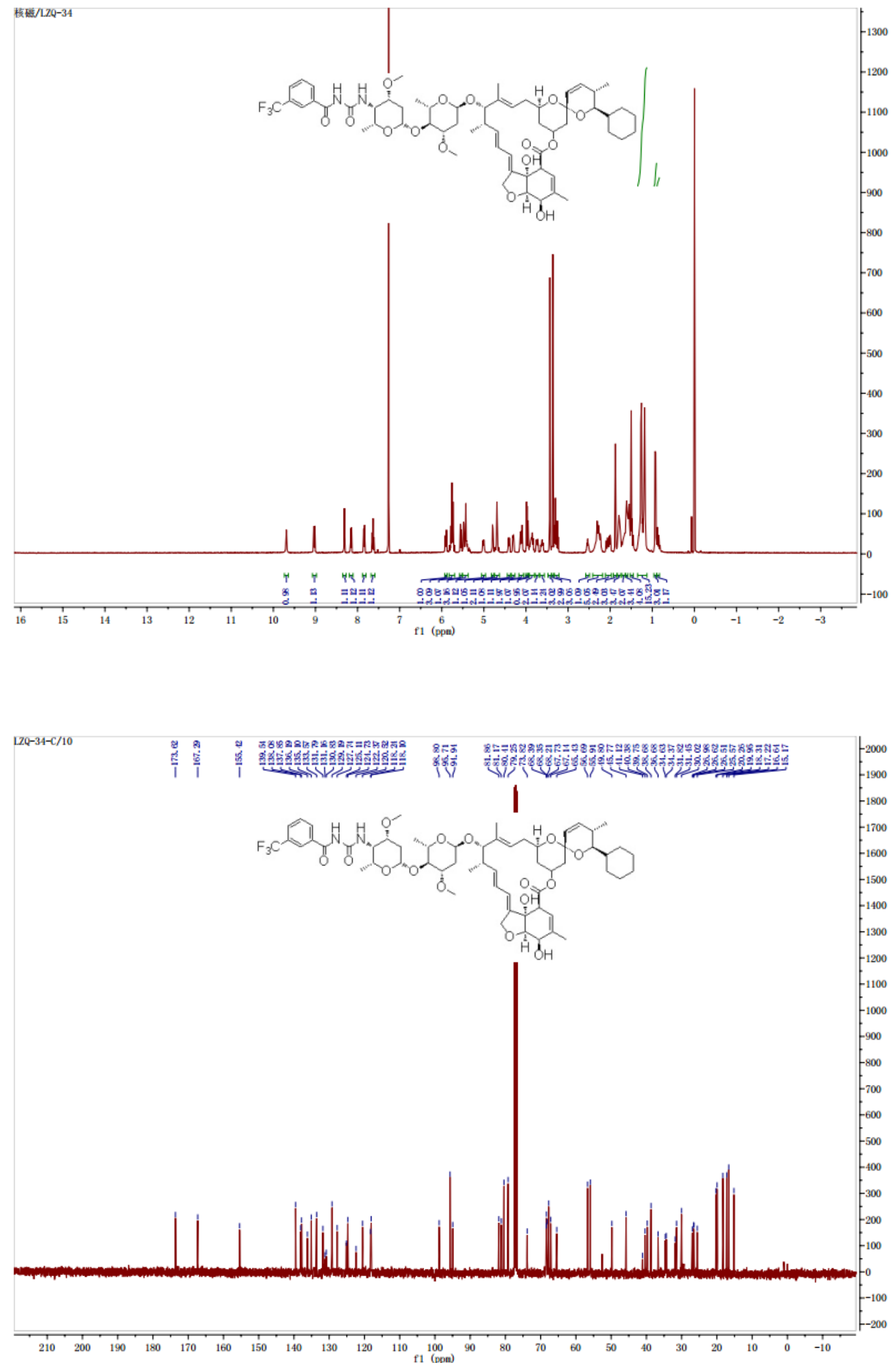
S-50: The ${ }^{1} \mathrm{H}-\mathrm{NMR}$ and ${ }^{13} \mathrm{C}-\mathrm{NMR}$ spectrums of $\mathbf{5 g}$
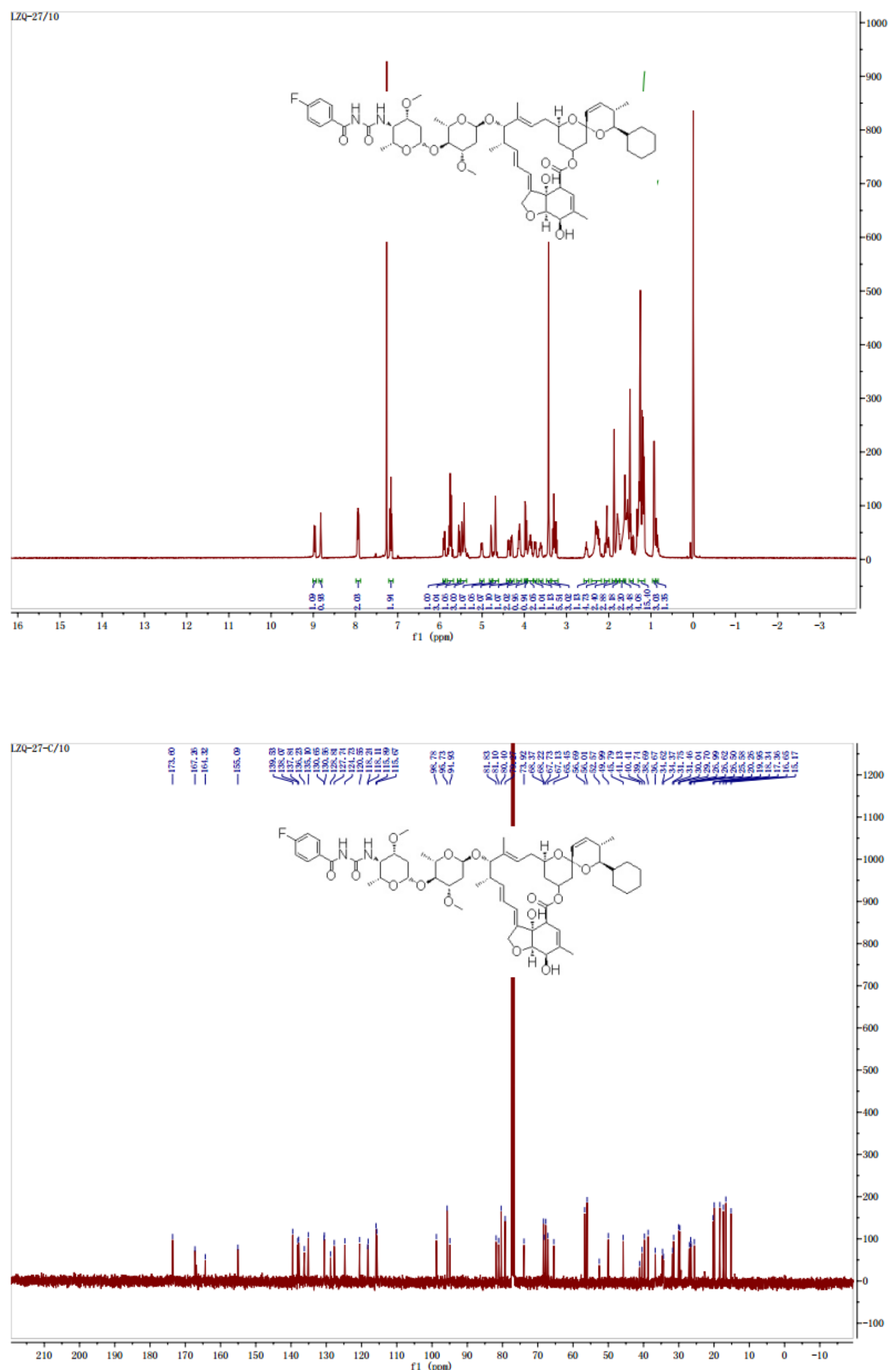
S-51: The ${ }^{1} \mathrm{H}-\mathrm{NMR}$ and ${ }^{13} \mathrm{C}-\mathrm{NMR}$ spectrums of $\mathbf{5 h}$
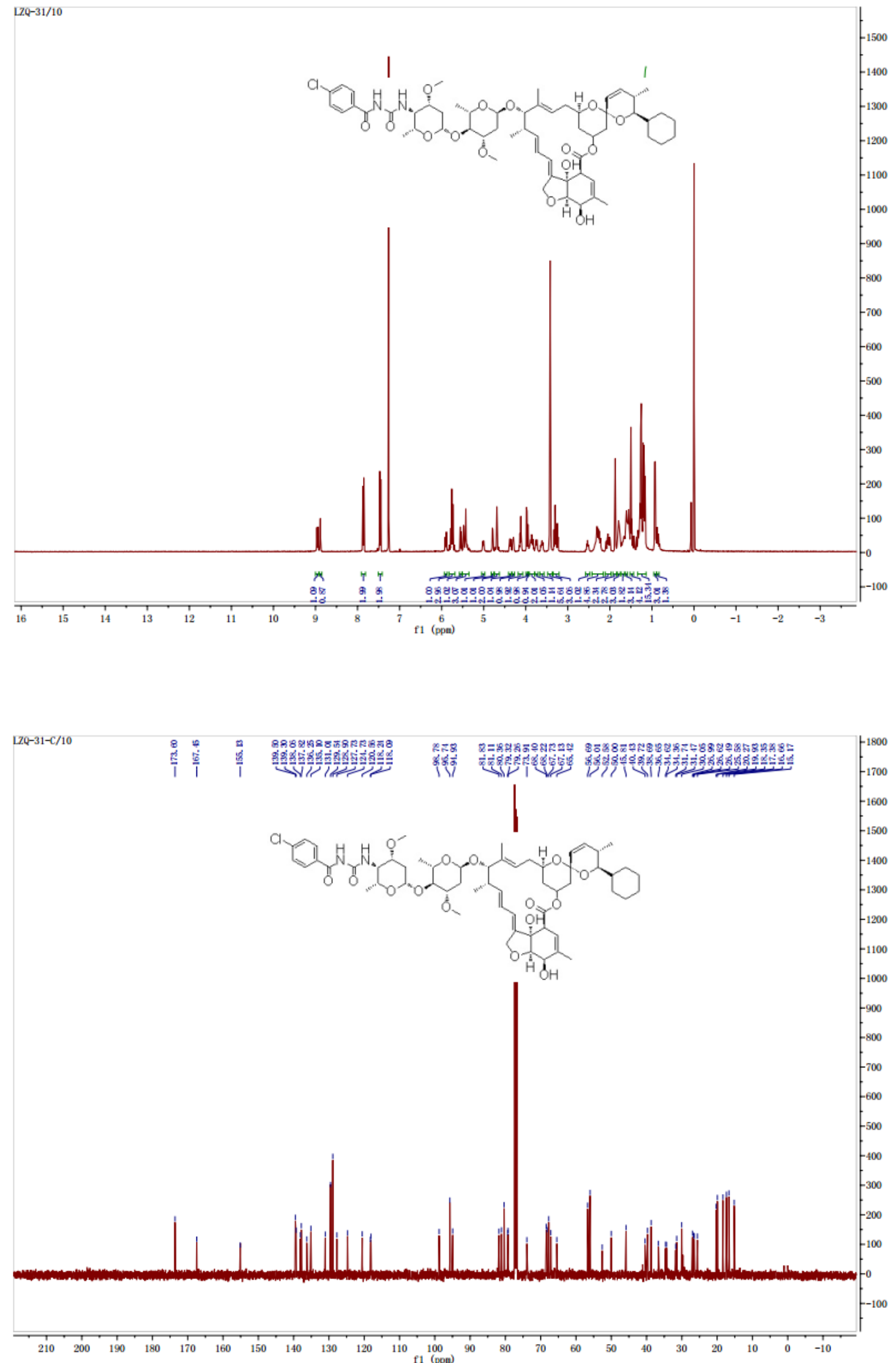
S-52: The ${ }^{1} \mathrm{H}-\mathrm{NMR}$ and ${ }^{13} \mathrm{C}-\mathrm{NMR}$ spectrums of $\mathbf{5 i}$
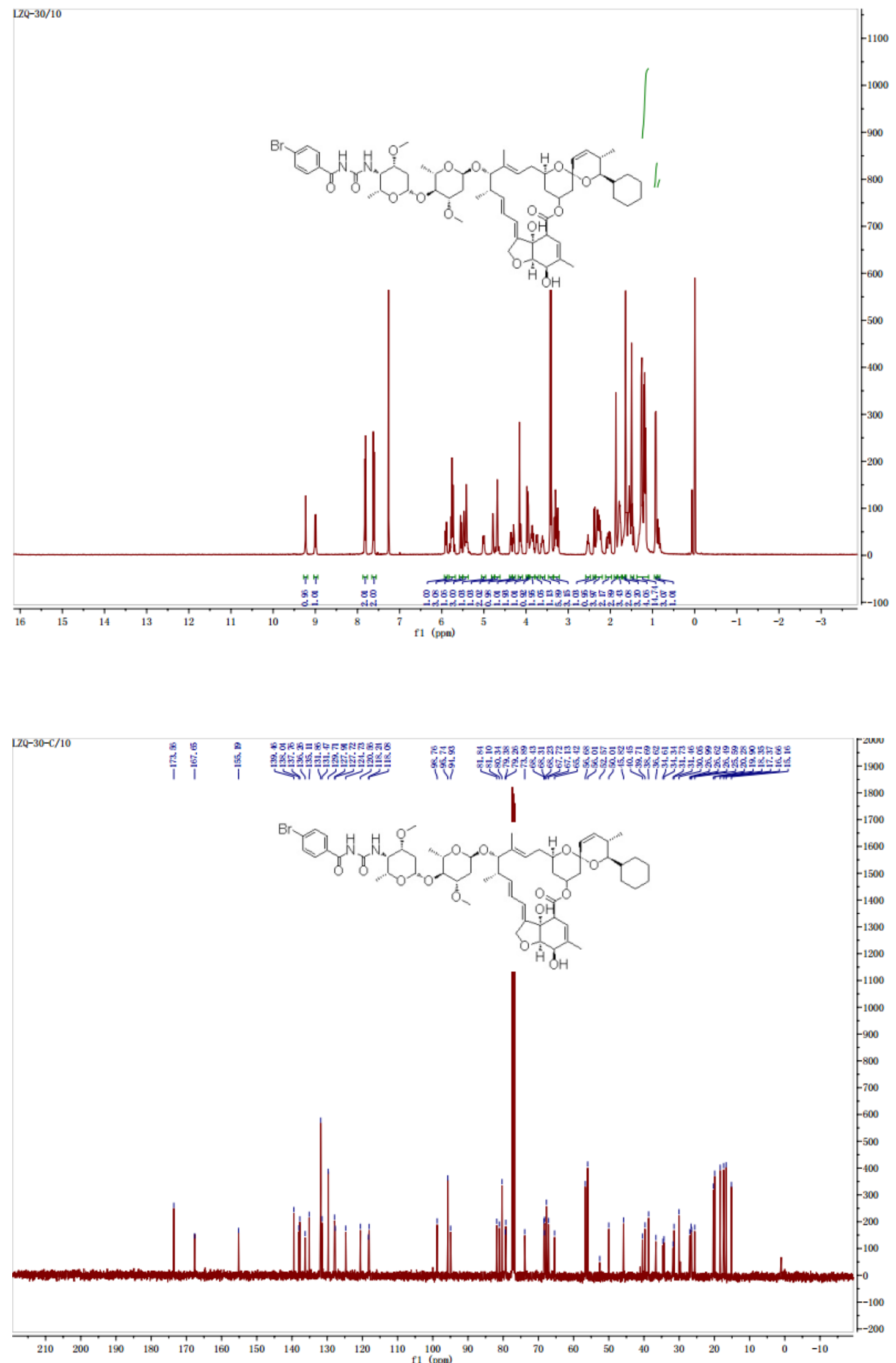
S-53: The ${ }^{1} \mathrm{H}-\mathrm{NMR}$ and ${ }^{13} \mathrm{C}-\mathrm{NMR}$ spectrums of $\mathbf{5 j}$
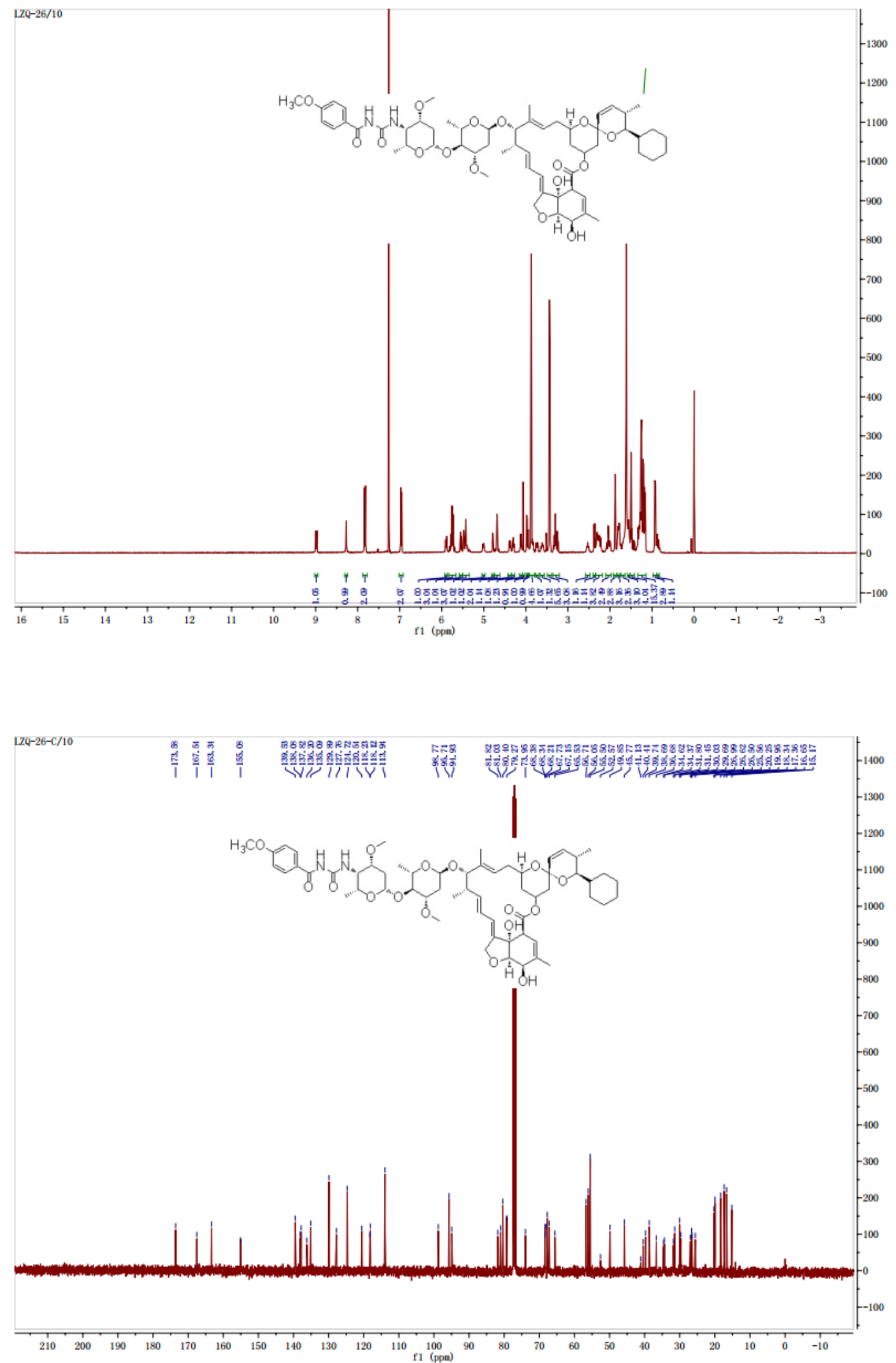
S-54: The ${ }^{1} \mathrm{H}-\mathrm{NMR}$ and ${ }^{13} \mathrm{C}-\mathrm{NMR}$ spectrums of $\mathbf{5 k}$
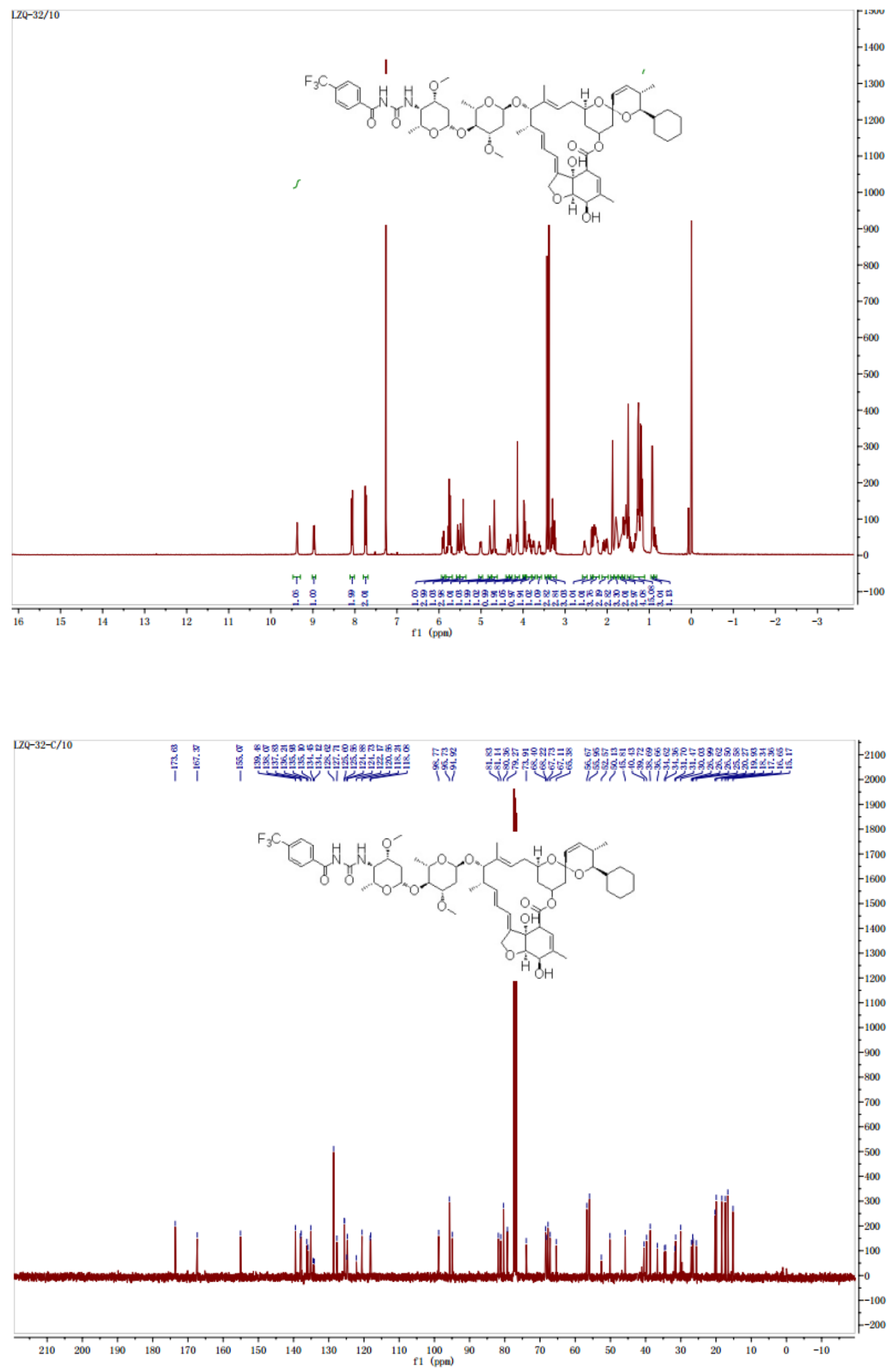
S-55: The ${ }^{1} \mathrm{H}-\mathrm{NMR}$ and ${ }^{13} \mathrm{C}-\mathrm{NMR}$ spectrums of $\mathbf{5 l}$
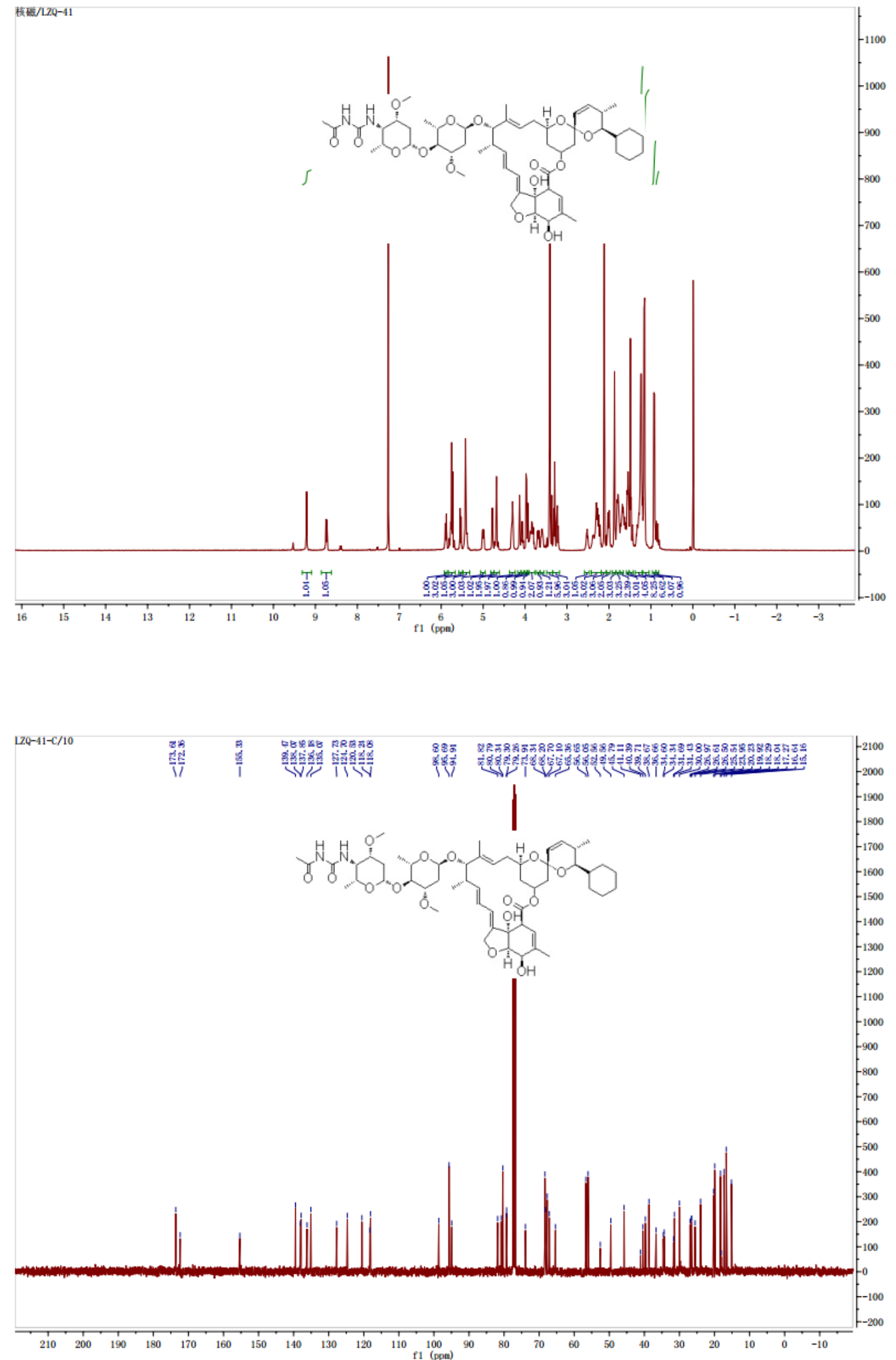
S-56: The ${ }^{1} \mathrm{H}-\mathrm{NMR}$ and ${ }^{13} \mathrm{C}-\mathrm{NMR}$ spectrums of $\mathbf{5 m}$
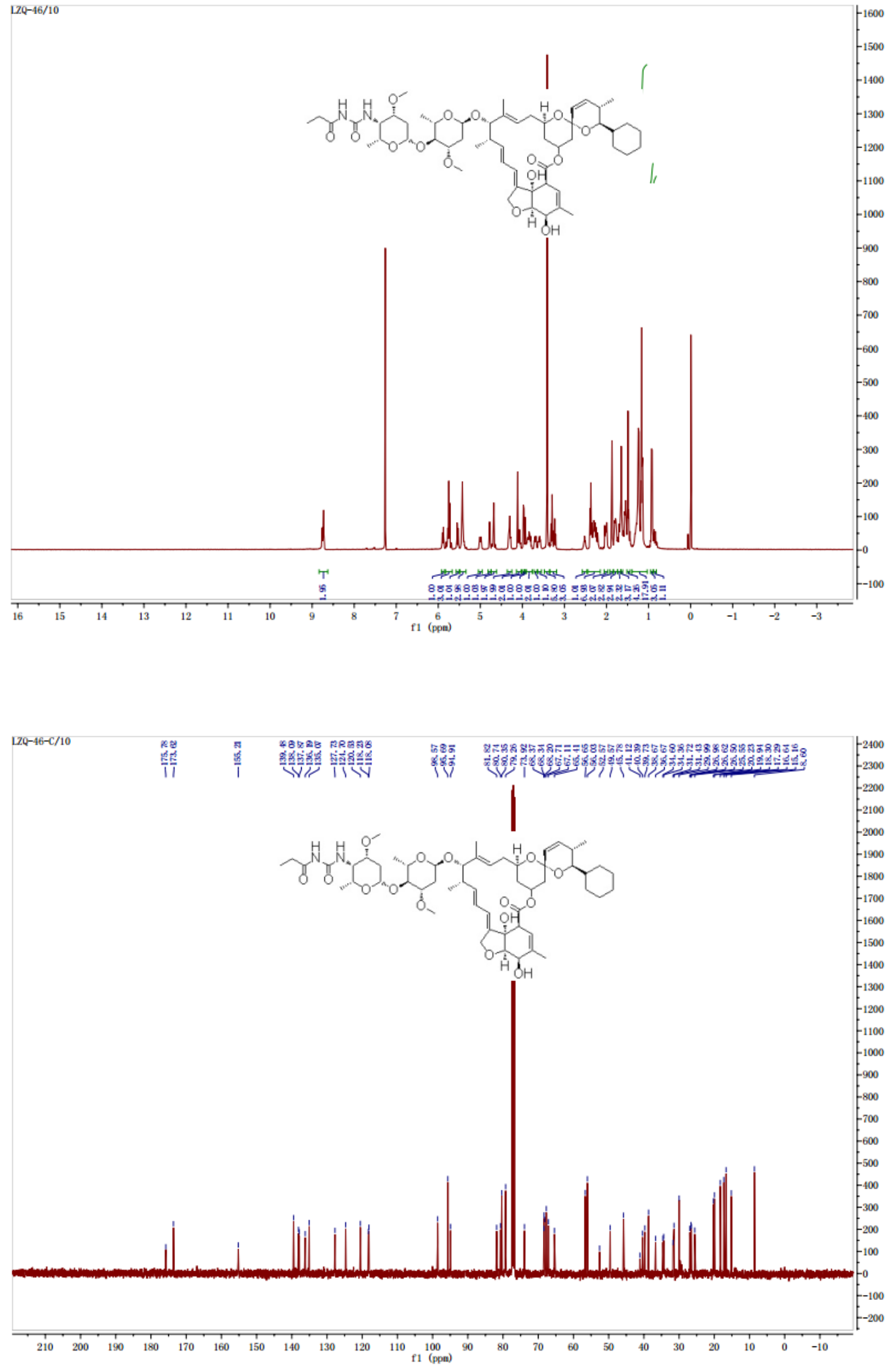
S-57: The ${ }^{1} \mathrm{H}-\mathrm{NMR}$ and ${ }^{13} \mathrm{C}-\mathrm{NMR}$ spectrums of $\mathbf{5 n}$
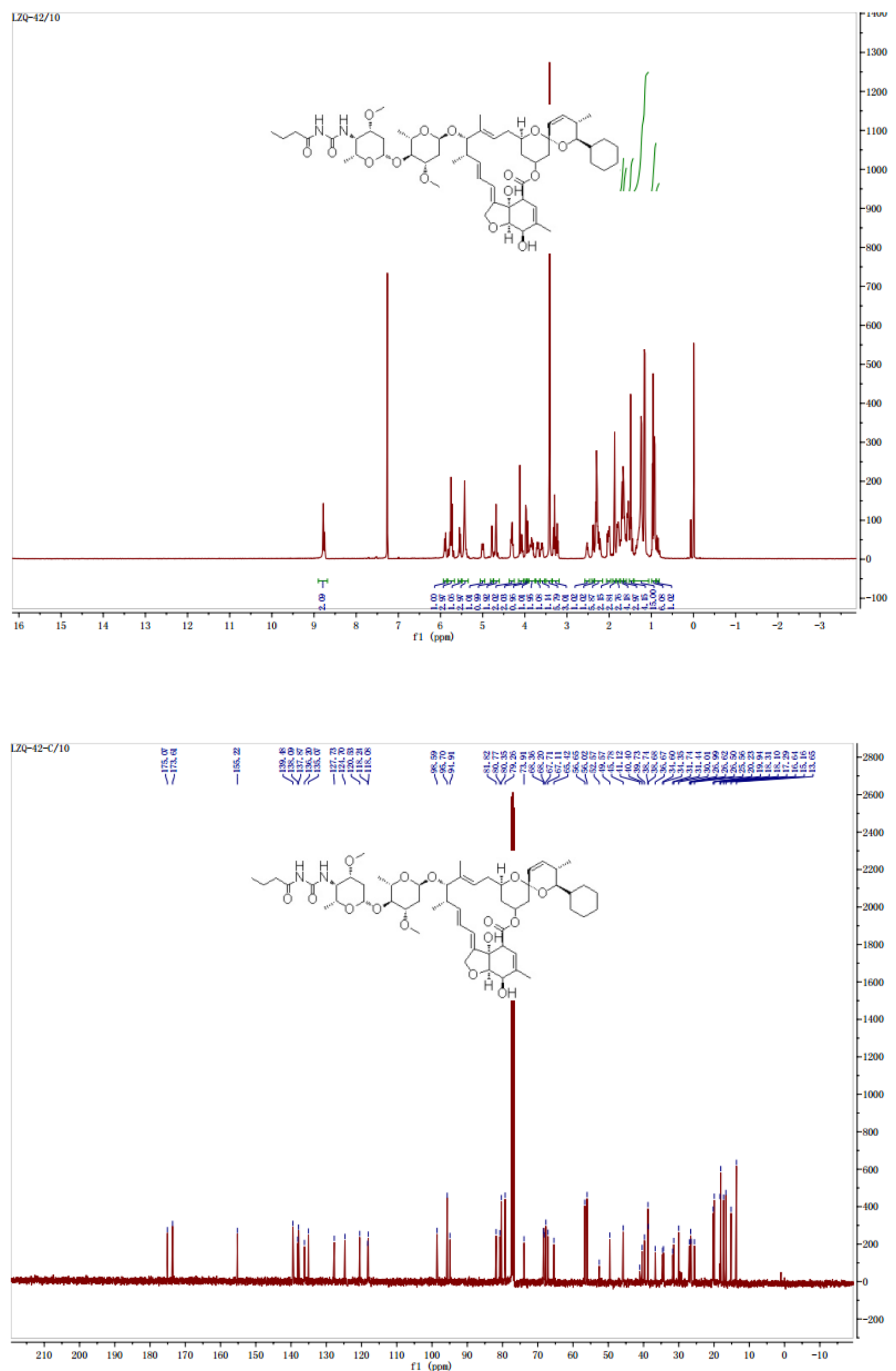
S-58: The ${ }^{1} \mathrm{H}-\mathrm{NMR}$ and ${ }^{13} \mathrm{C}-\mathrm{NMR}$ spectrums of 50
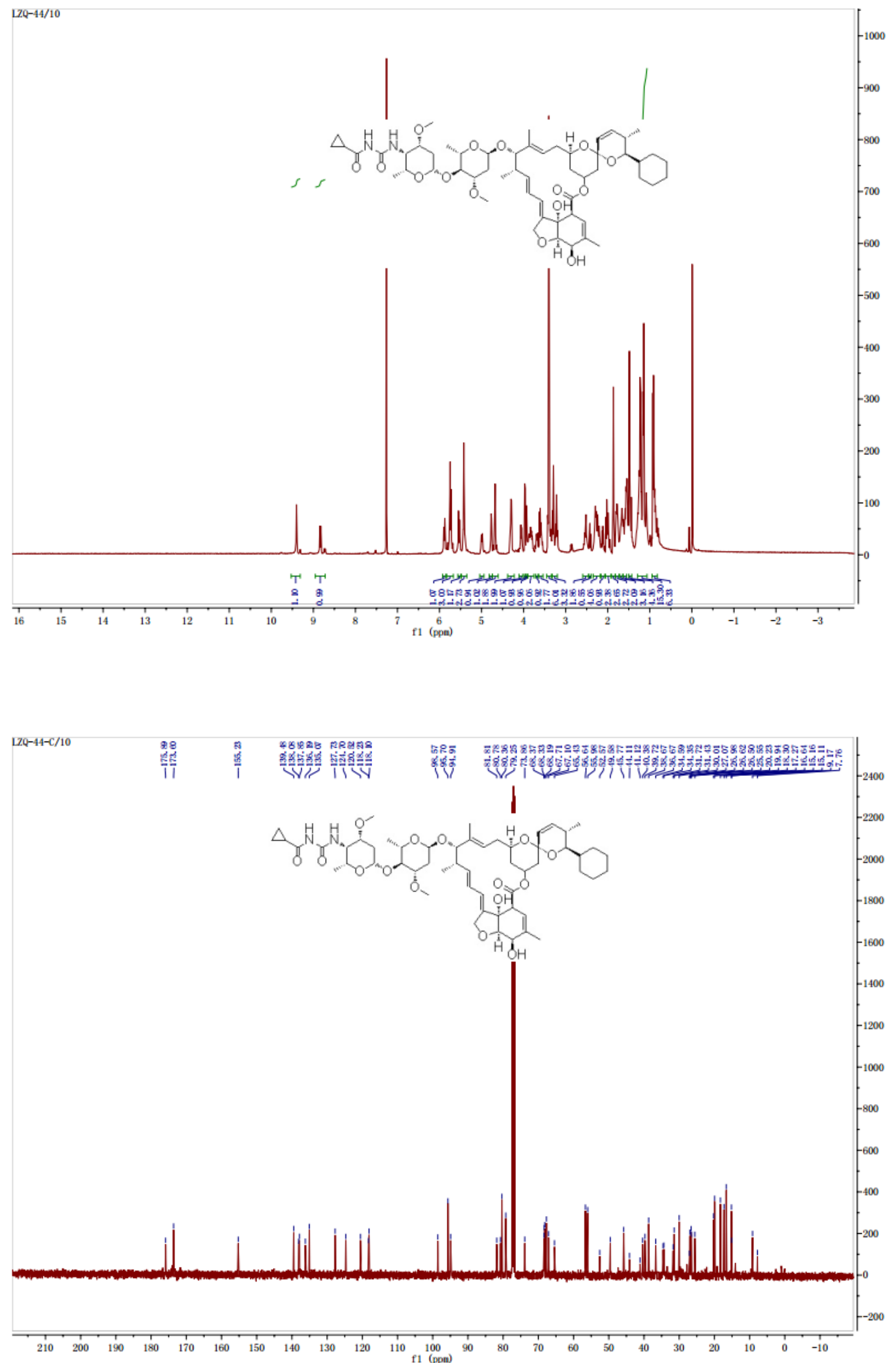
S-59: The ${ }^{1} \mathrm{H}-\mathrm{NMR}$ and ${ }^{13} \mathrm{C}-\mathrm{NMR}$ spectrums of $\mathbf{5 p}$
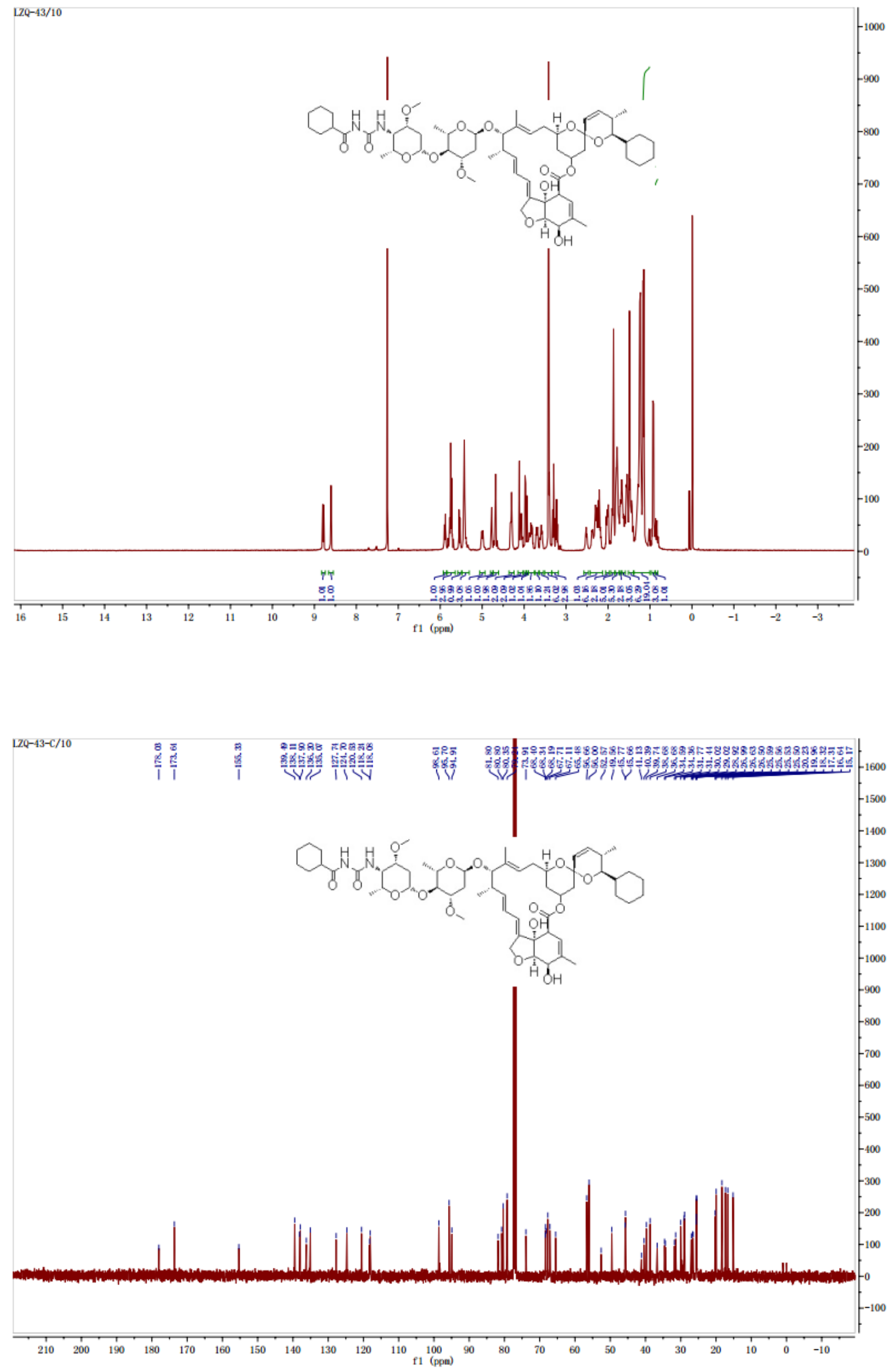
S-60: The ${ }^{1} \mathrm{H}-\mathrm{NMR}$ and ${ }^{13} \mathrm{C}-\mathrm{NMR}$ spectrums of $\mathbf{5 q}$
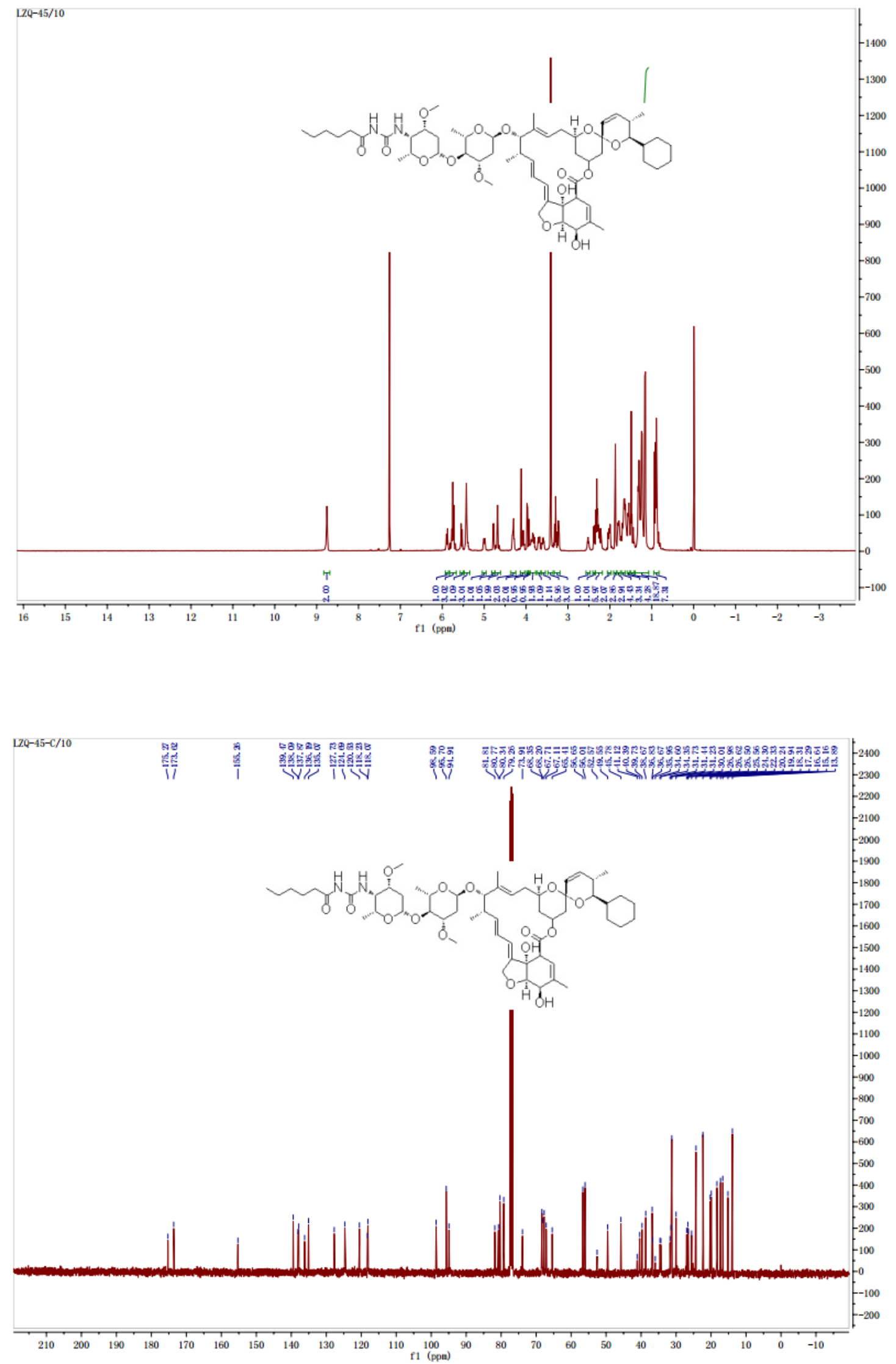
S-61: The ${ }^{1} \mathrm{H}-\mathrm{NMR}$ and ${ }^{13} \mathrm{C}-\mathrm{NMR}$ spectrums of $\mathbf{5 r}$
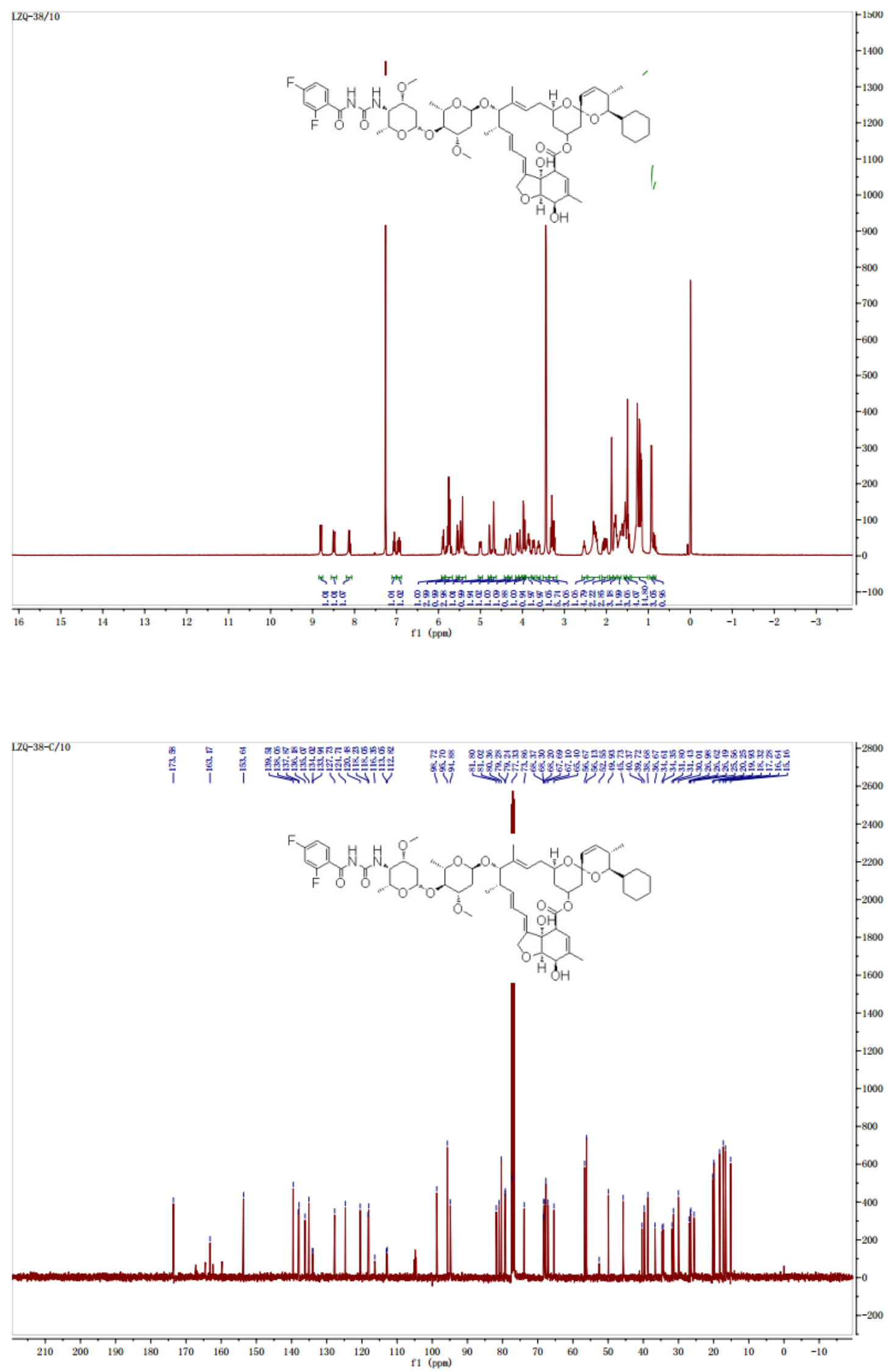
S-62: The ${ }^{1} \mathrm{H}-\mathrm{NMR}$ and ${ }^{13} \mathrm{C}-\mathrm{NMR}$ spectrums of $\mathbf{5 s}$
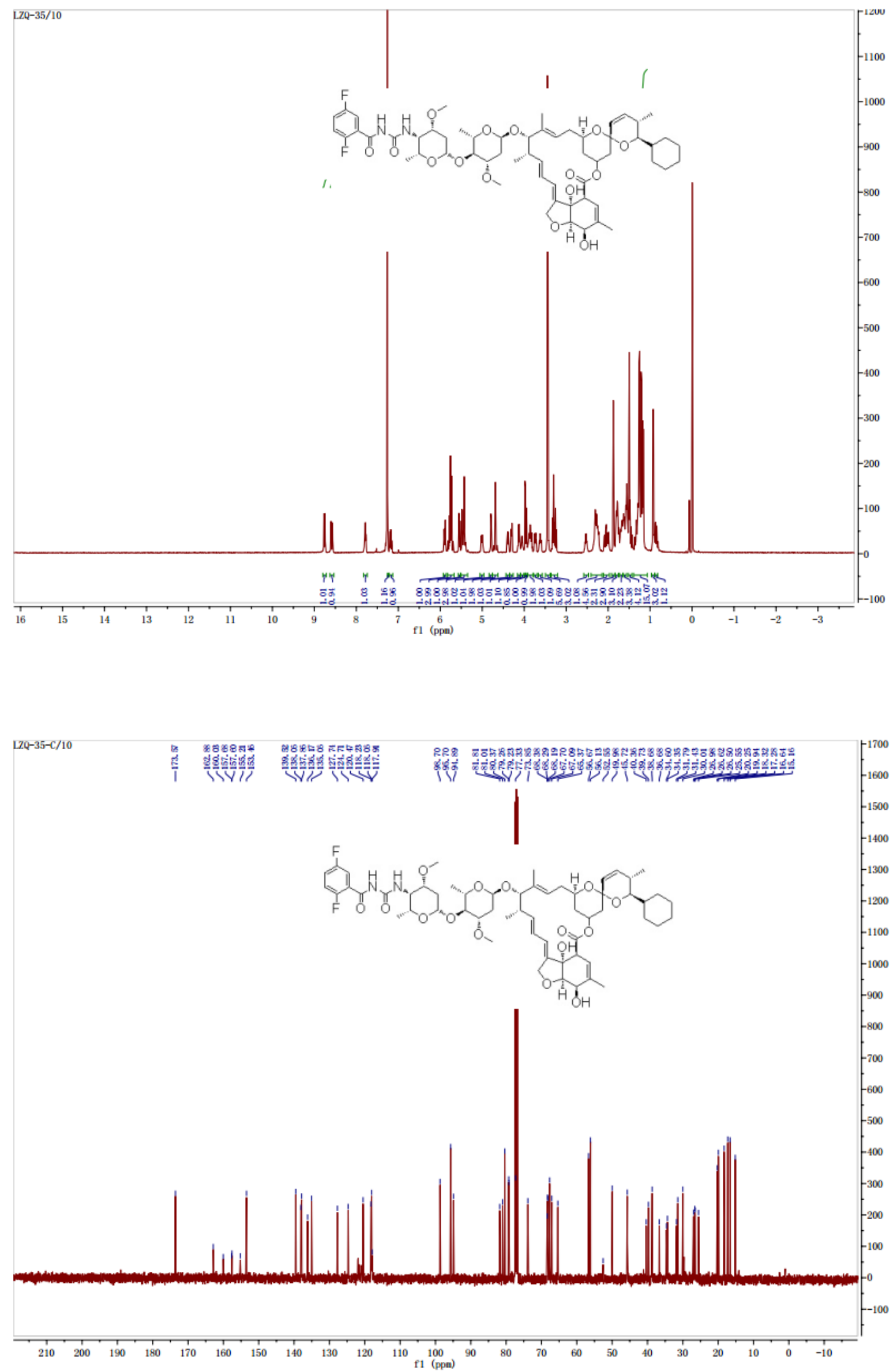
S-63: The ${ }^{1} \mathrm{H}-\mathrm{NMR}$ and ${ }^{13} \mathrm{C}-\mathrm{NMR}$ spectrums of $\mathbf{5 t}$
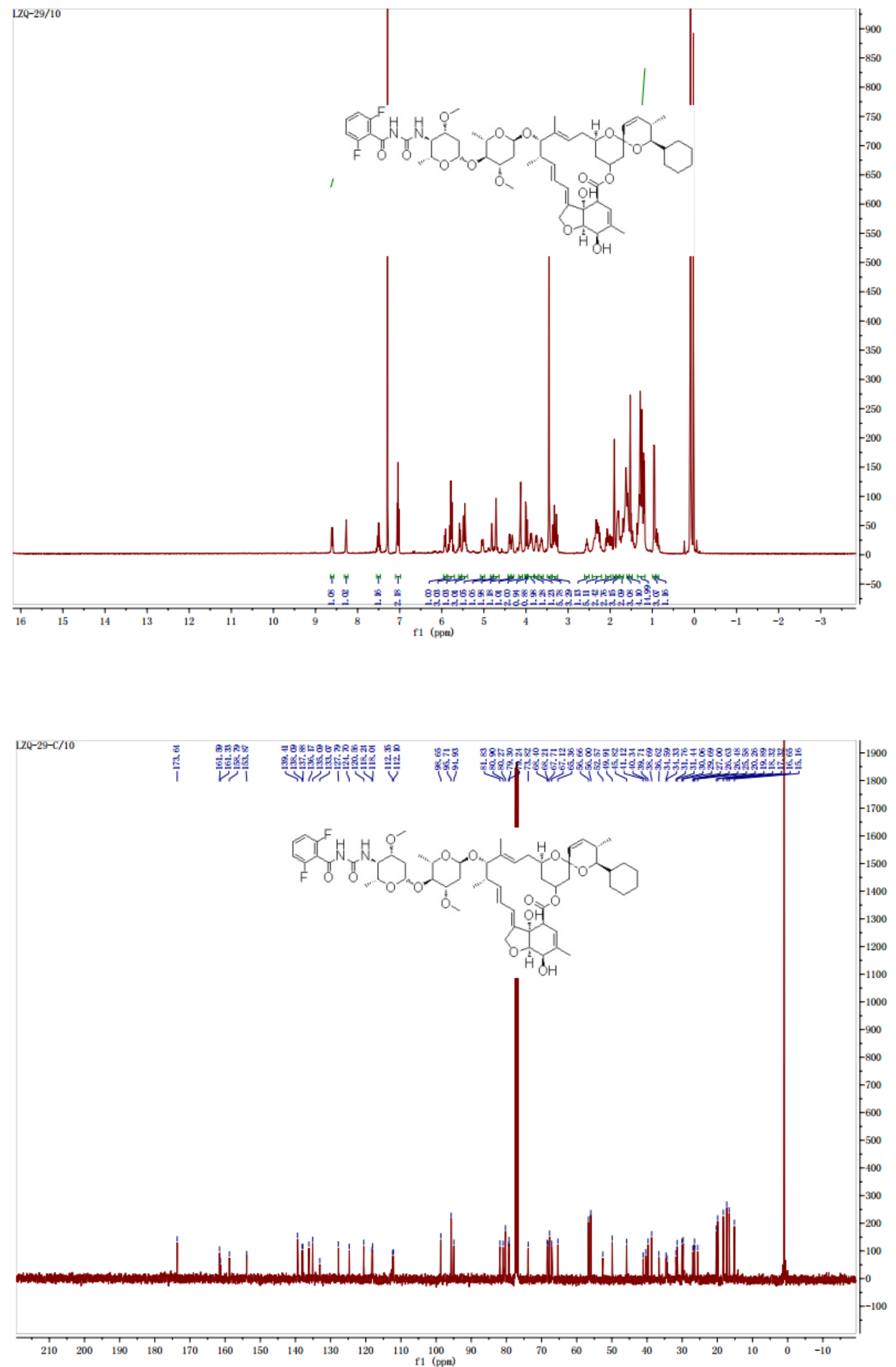
S-64: The ${ }^{1} \mathrm{H}-\mathrm{NMR}$ and ${ }^{13} \mathrm{C}-\mathrm{NMR}$ spectrums of $\mathbf{5 u}$
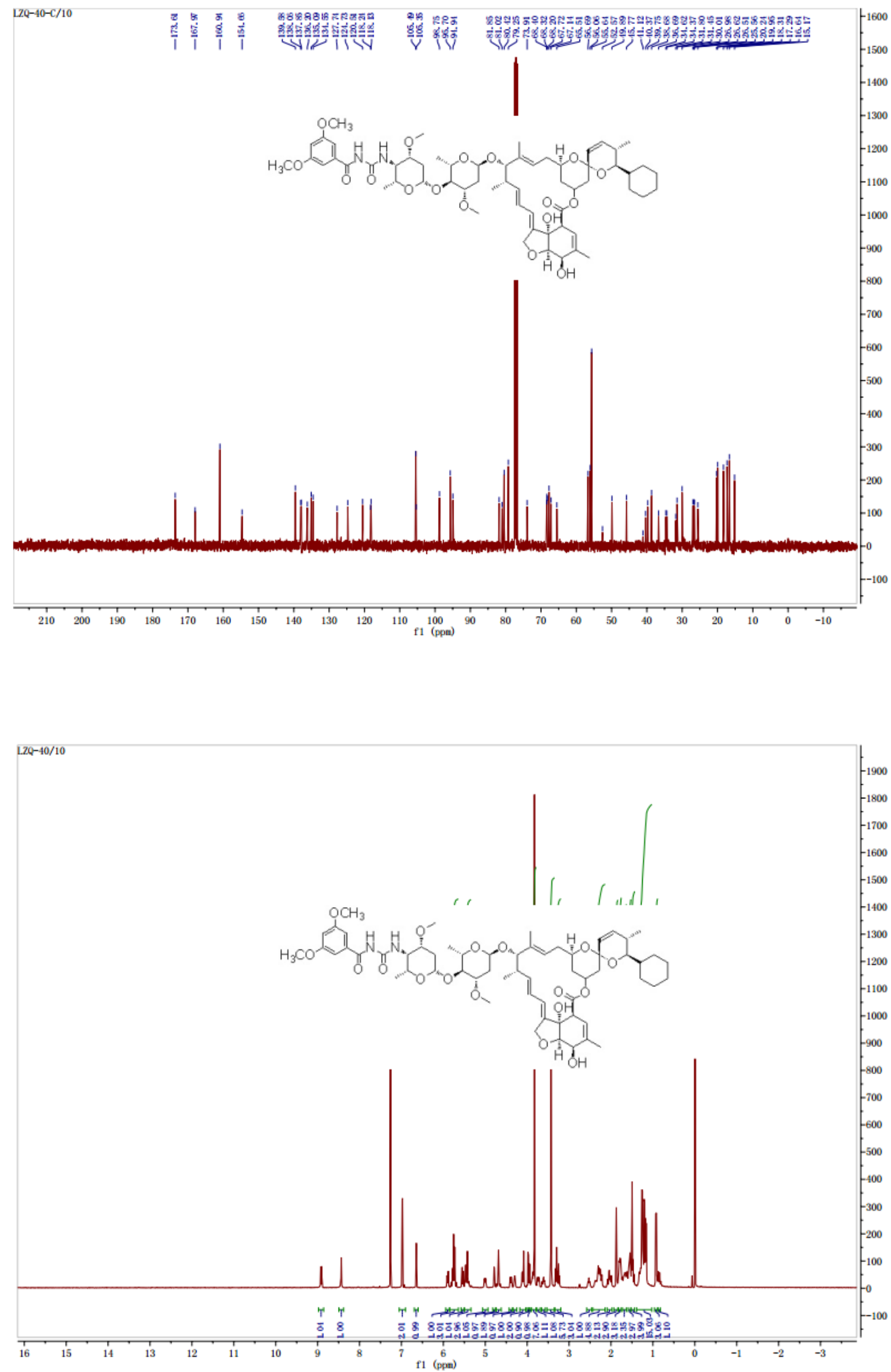
S-65: The ${ }^{1} \mathrm{H}-\mathrm{NMR}$ and ${ }^{13} \mathrm{C}-\mathrm{NMR}$ spectrums of $\mathbf{5 v}$
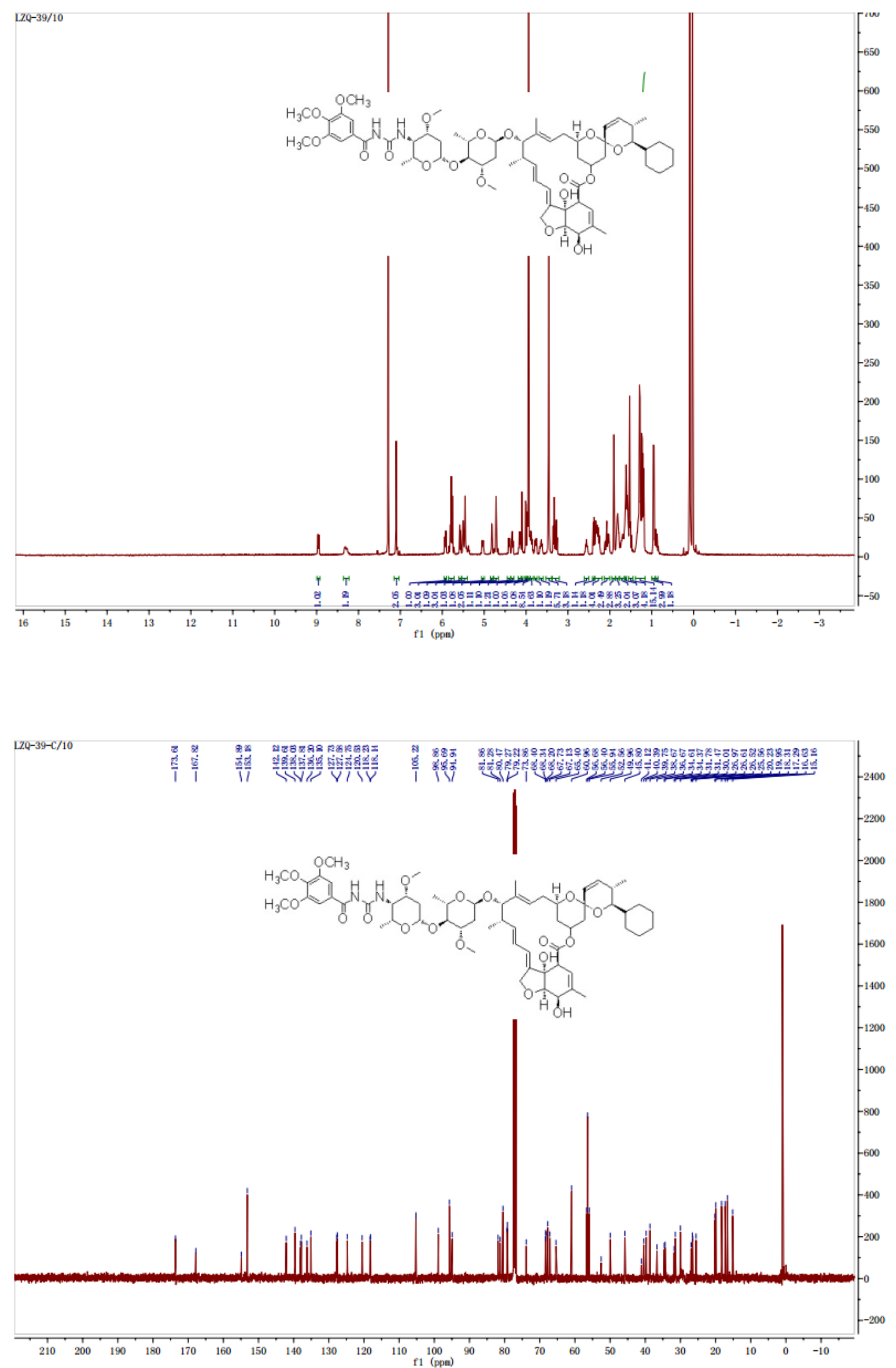
S-66: The ${ }^{1} \mathrm{H}-\mathrm{NMR}$ and ${ }^{13} \mathrm{C}-\mathrm{NMR}$ spectrums of $\mathbf{6 a}$
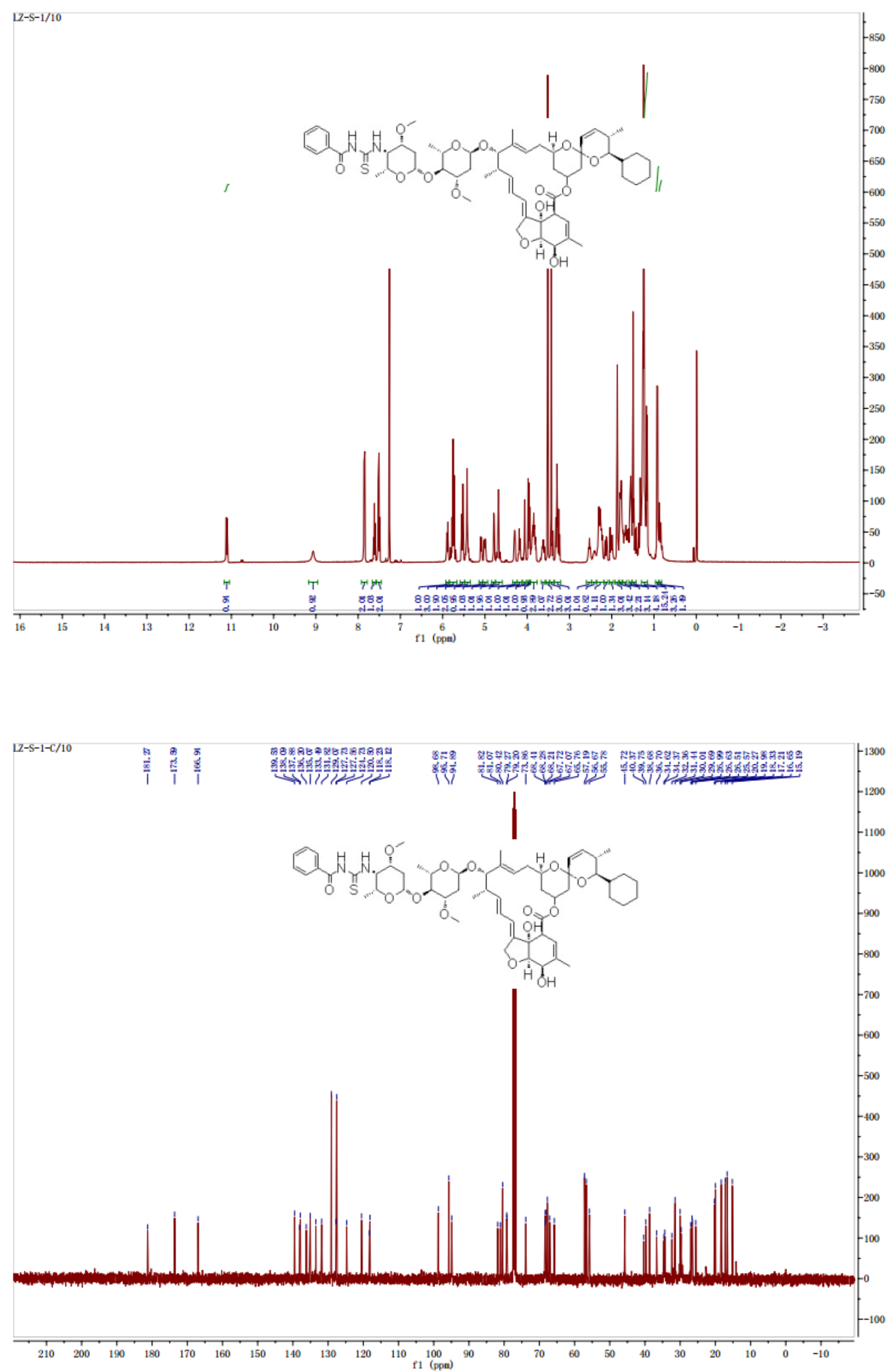
S-67: The ${ }^{1} \mathrm{H}-\mathrm{NMR}$ and ${ }^{13} \mathrm{C}-\mathrm{NMR}$ spectrums of $\mathbf{6 b}$
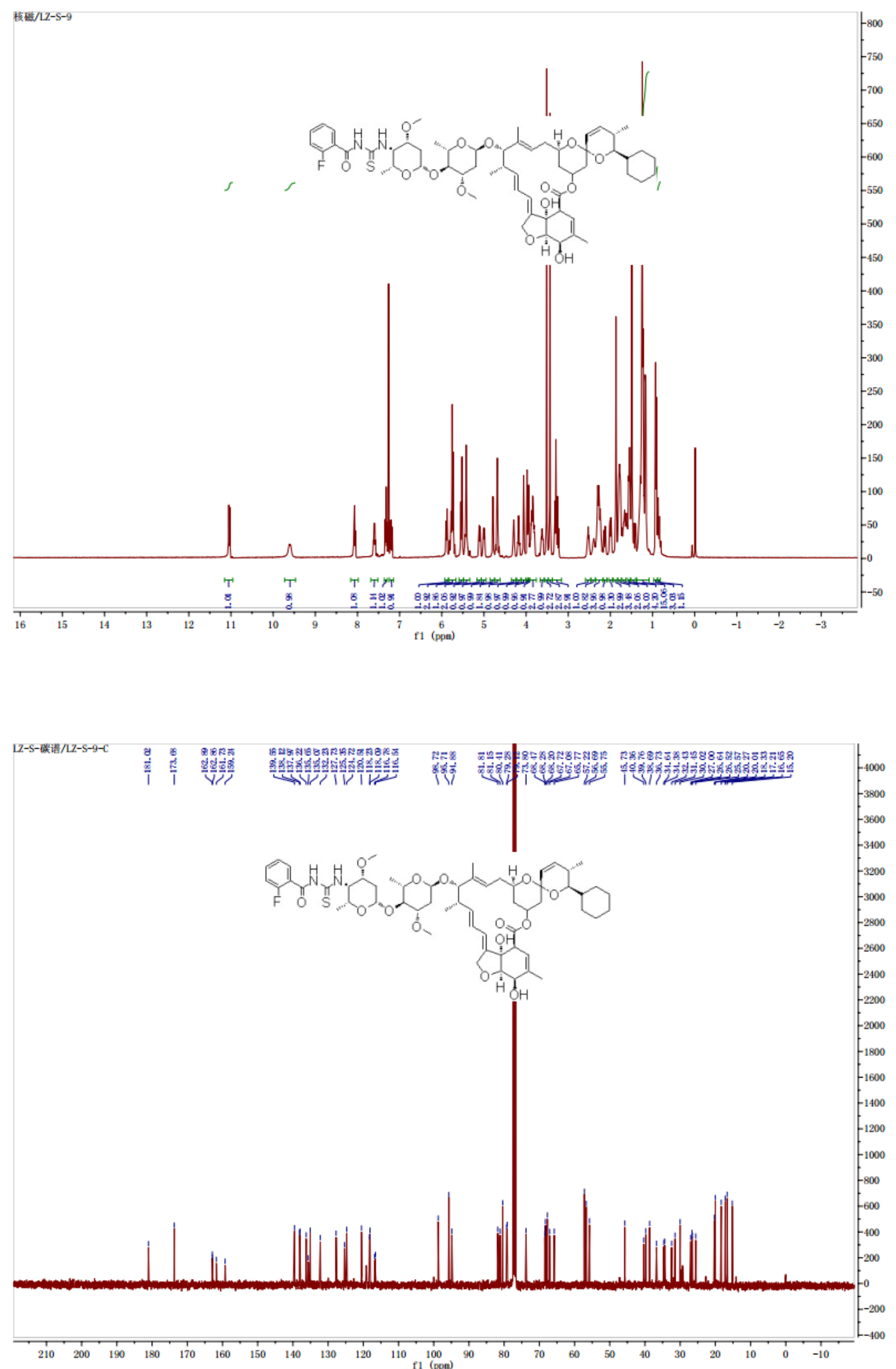
S-68: The ${ }^{1} \mathrm{H}-\mathrm{NMR}$ and ${ }^{13} \mathrm{C}-\mathrm{NMR}$ spectrums of $\mathbf{6 c}$
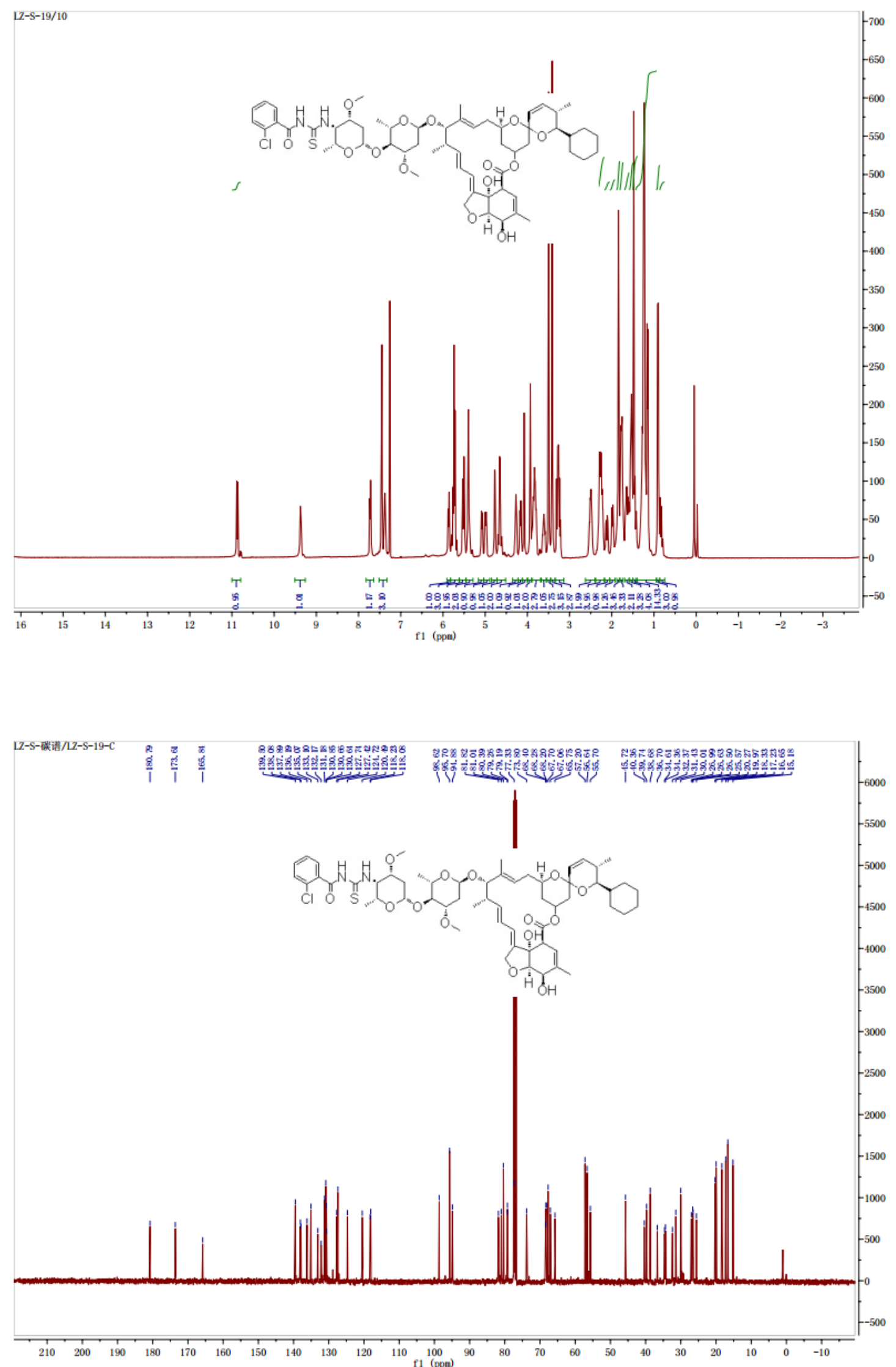
S-69: The ${ }^{1} \mathrm{H}-\mathrm{NMR}$ and ${ }^{13} \mathrm{C}-\mathrm{NMR}$ spectrums of $\mathbf{6 d}$
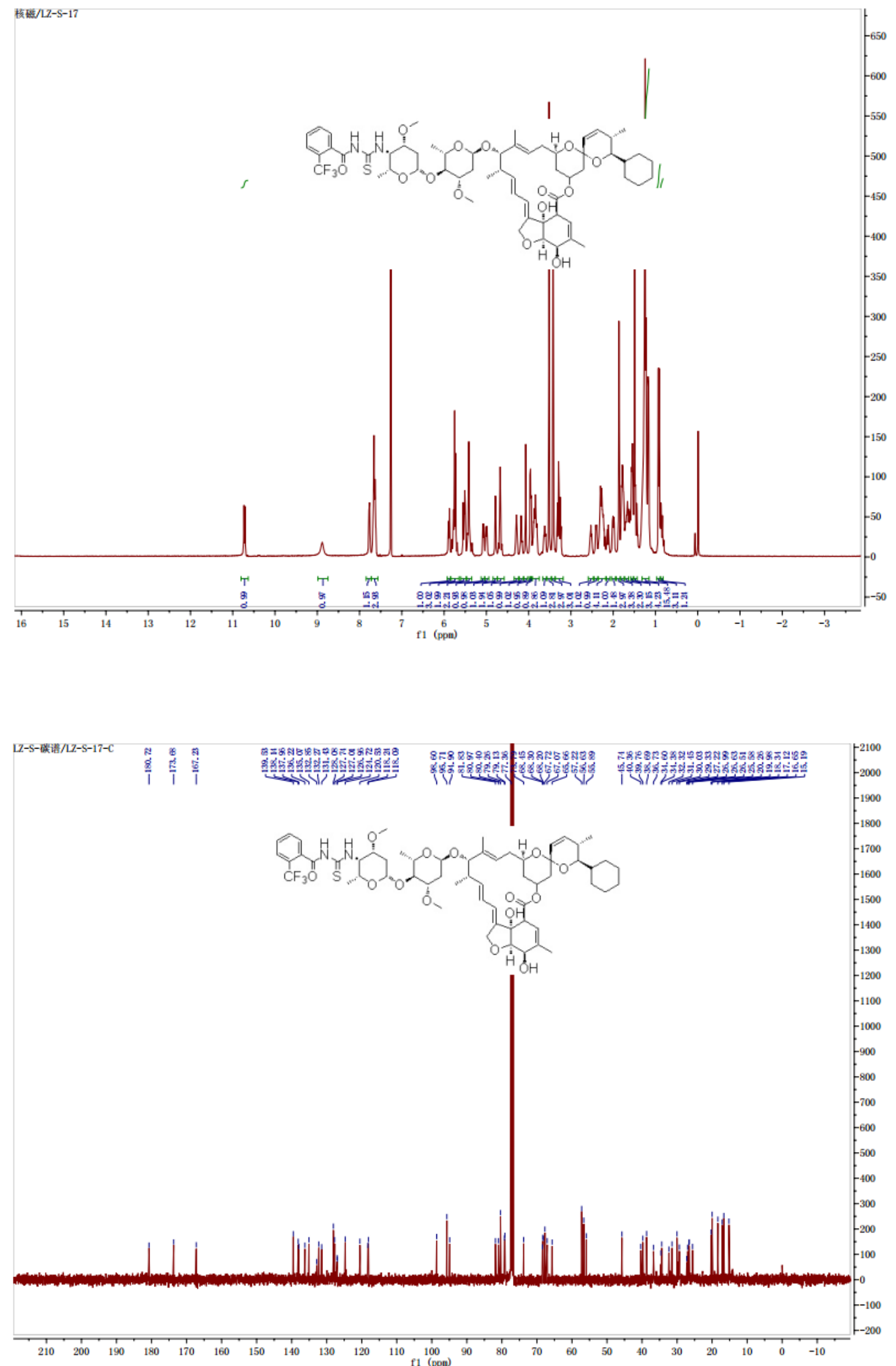
S-70: The ${ }^{1} \mathrm{H}-\mathrm{NMR}$ and ${ }^{13} \mathrm{C}-\mathrm{NMR}$ spectrums of $\mathbf{6 e}$
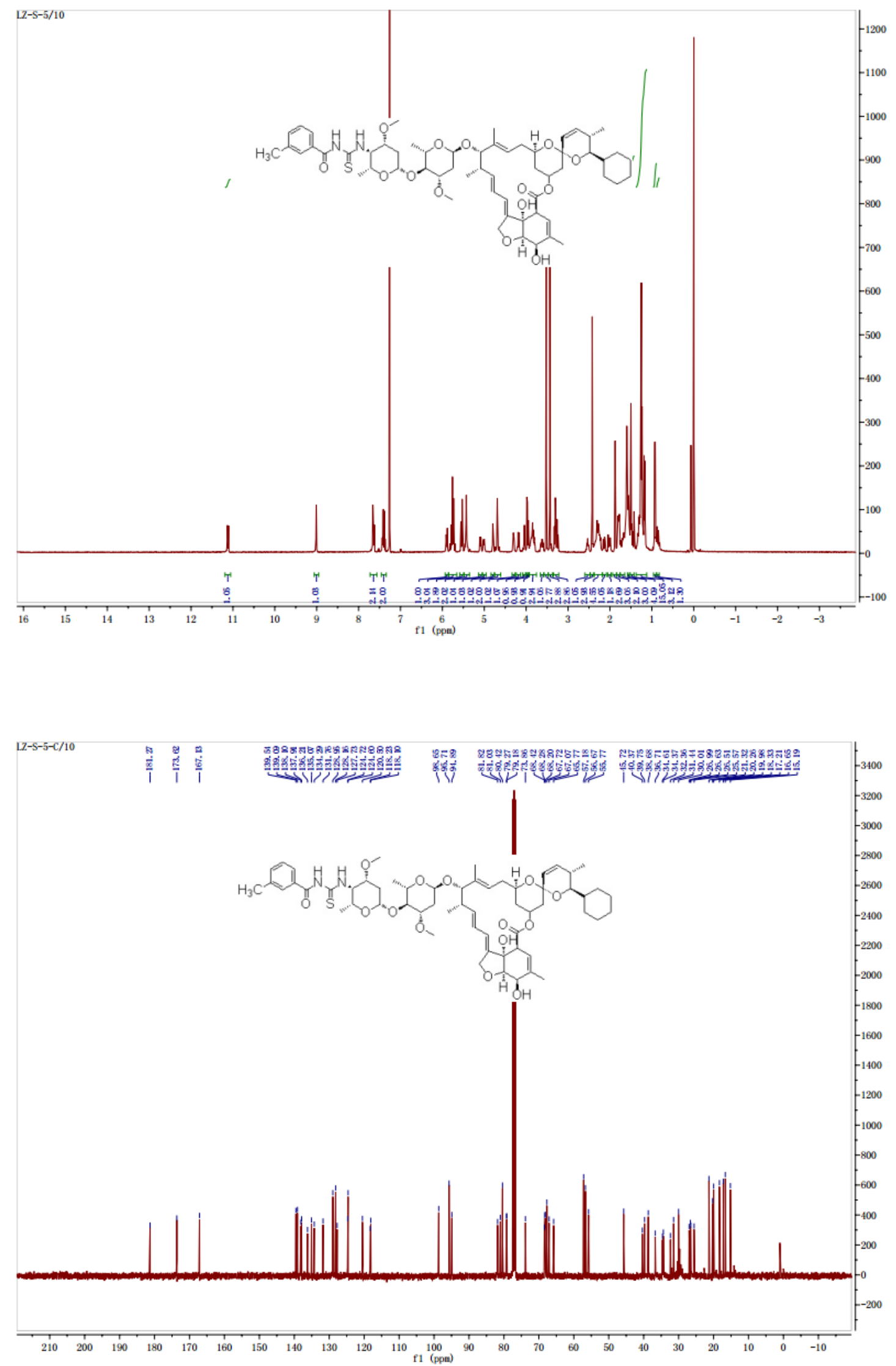
S-71: The ${ }^{1} \mathrm{H}-\mathrm{NMR}$ and ${ }^{13} \mathrm{C}-\mathrm{NMR}$ spectrums of $\mathbf{6 f}$
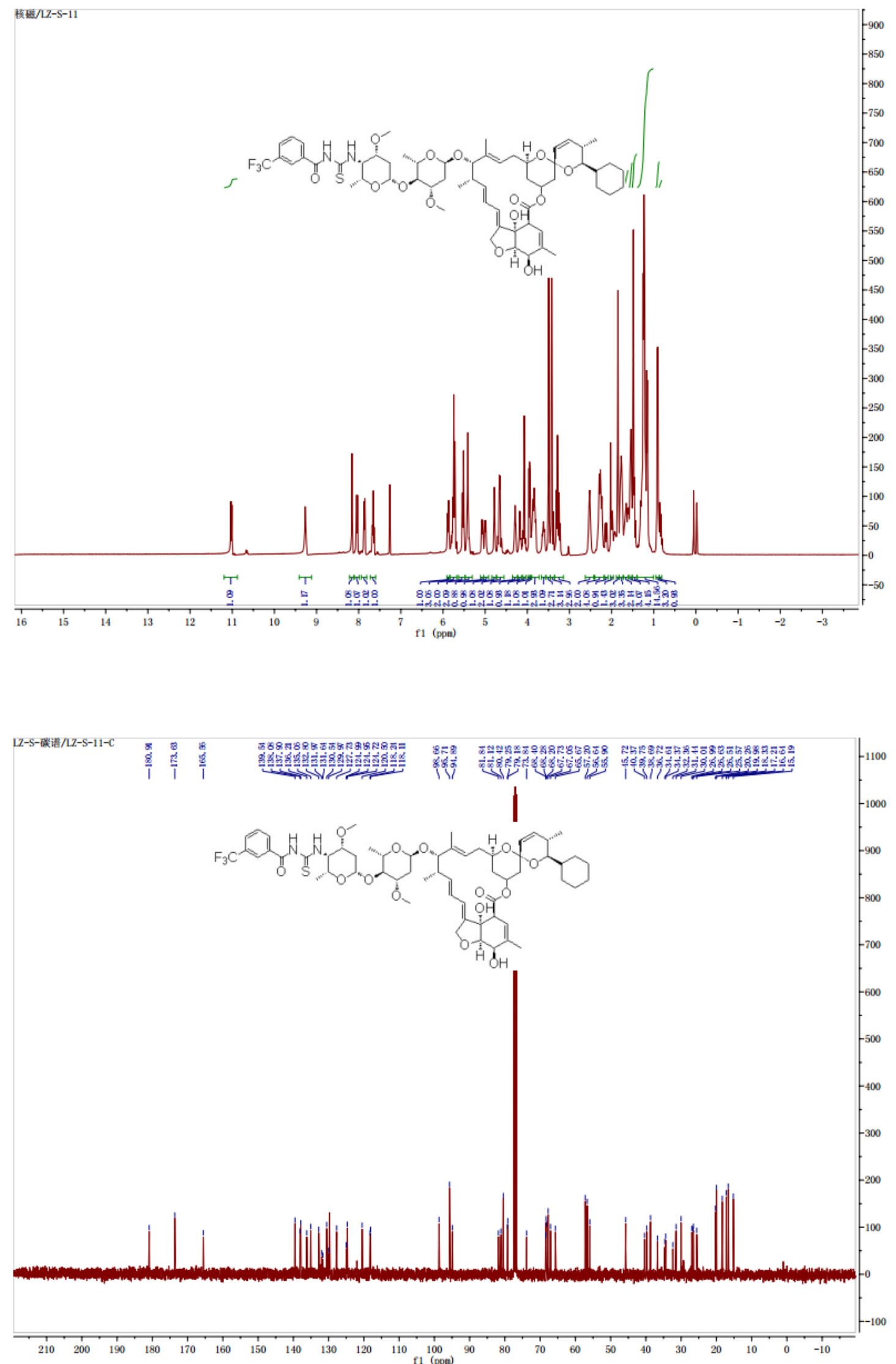
S-72: The ${ }^{1} \mathrm{H}-\mathrm{NMR}$ and ${ }^{13} \mathrm{C}-\mathrm{NMR}$ spectrums of $\mathbf{6 g}$
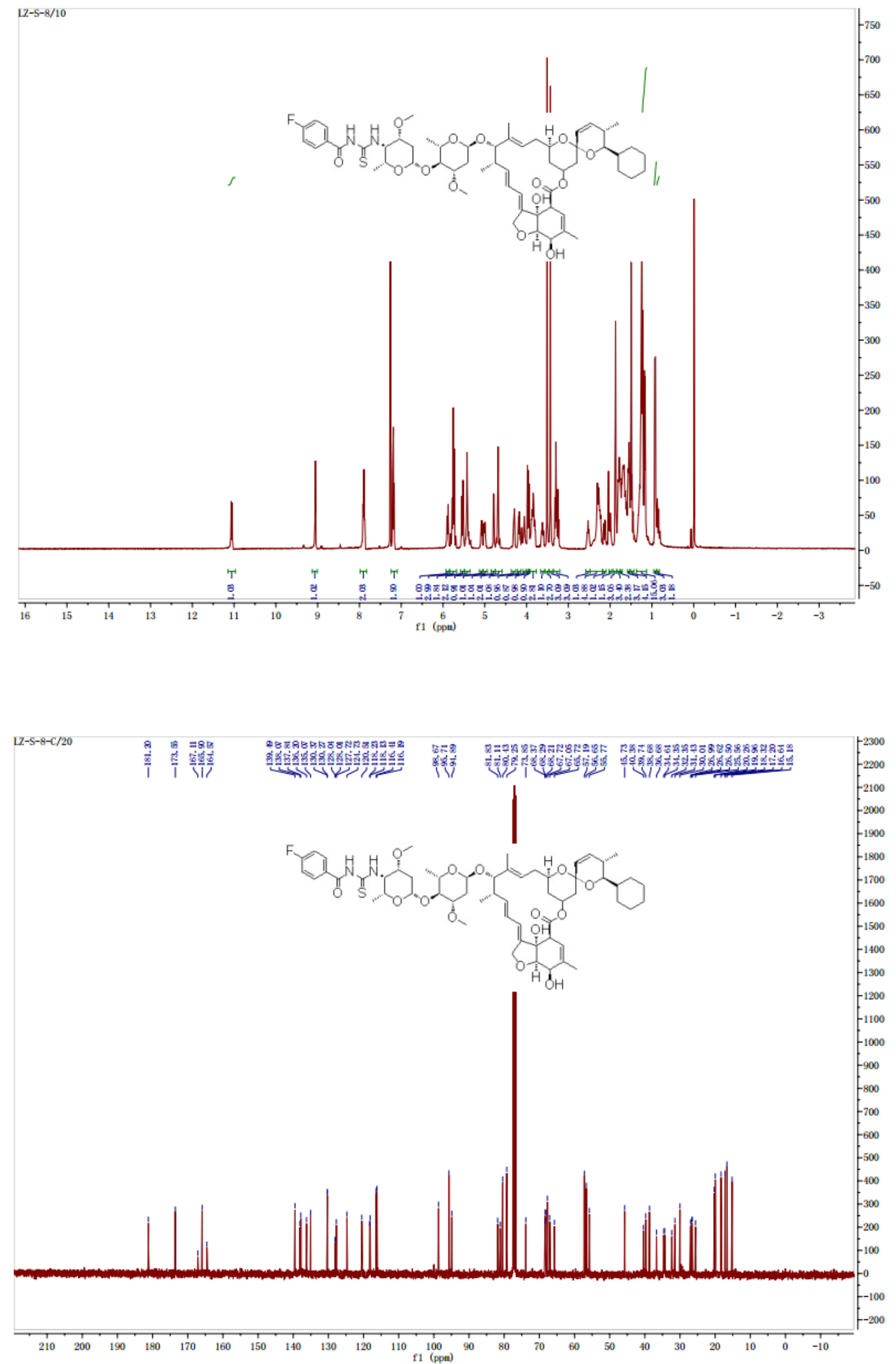
S-73: The ${ }^{1} \mathrm{H}-\mathrm{NMR}$ and ${ }^{13} \mathrm{C}-\mathrm{NMR}$ spectrums of $\mathbf{6 h}$
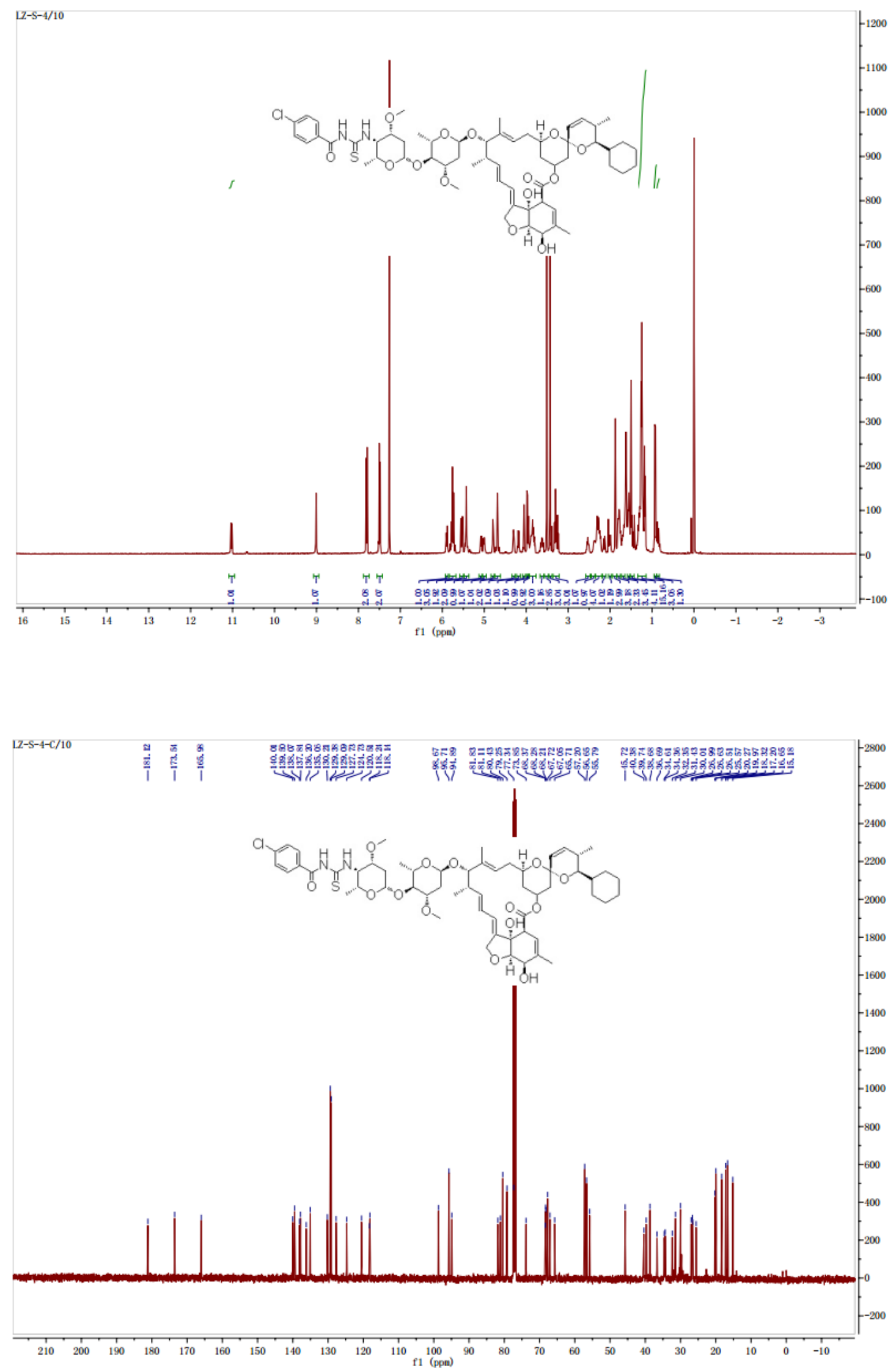
S-74: The ${ }^{1} \mathrm{H}-\mathrm{NMR}$ and ${ }^{13} \mathrm{C}-\mathrm{NMR}$ spectrums of $\mathbf{6 i}$
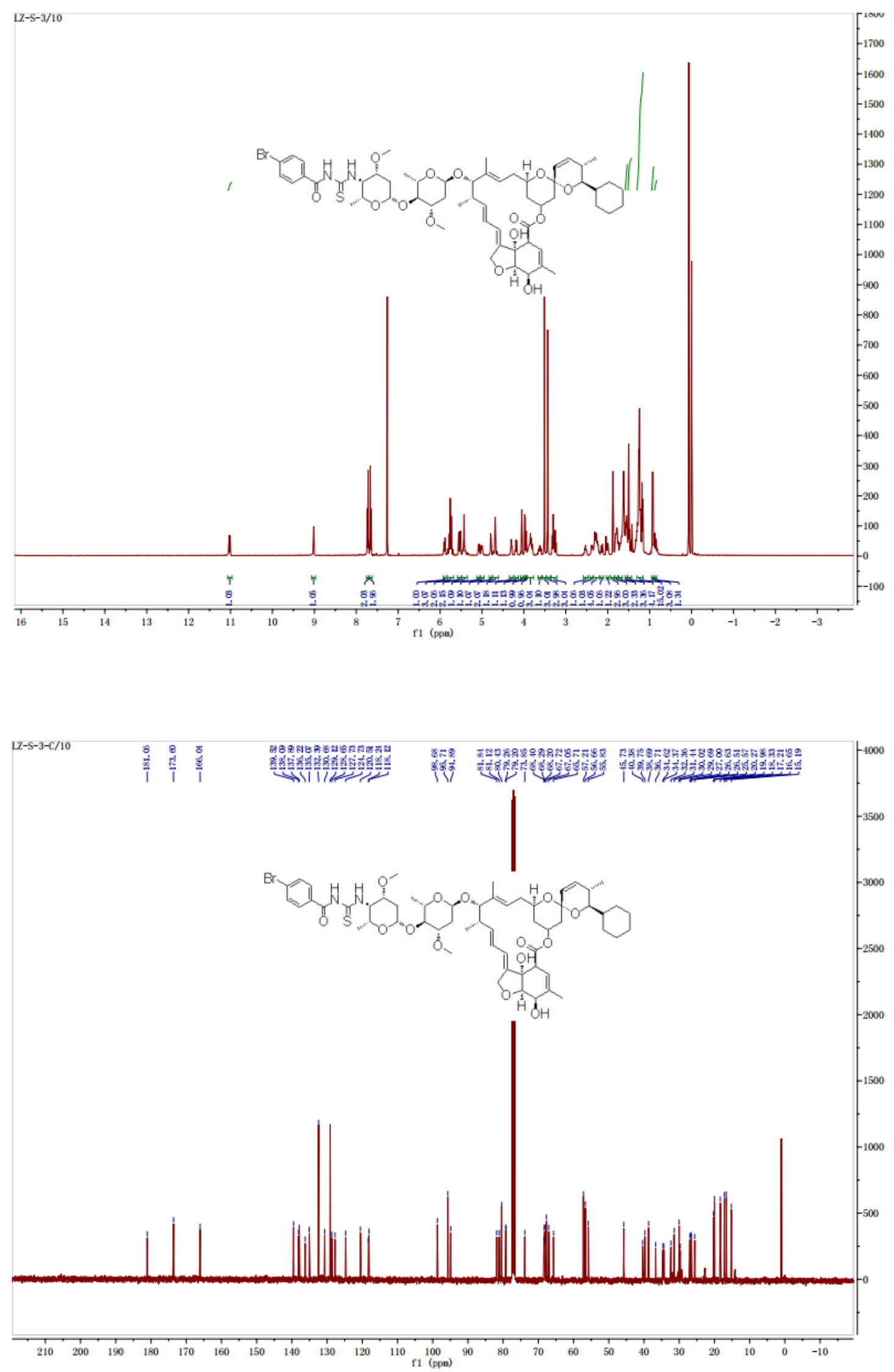
S-75: The ${ }^{1} \mathrm{H}-\mathrm{NMR}$ and ${ }^{13} \mathrm{C}-\mathrm{NMR}$ spectrums of $\mathbf{6 j}$
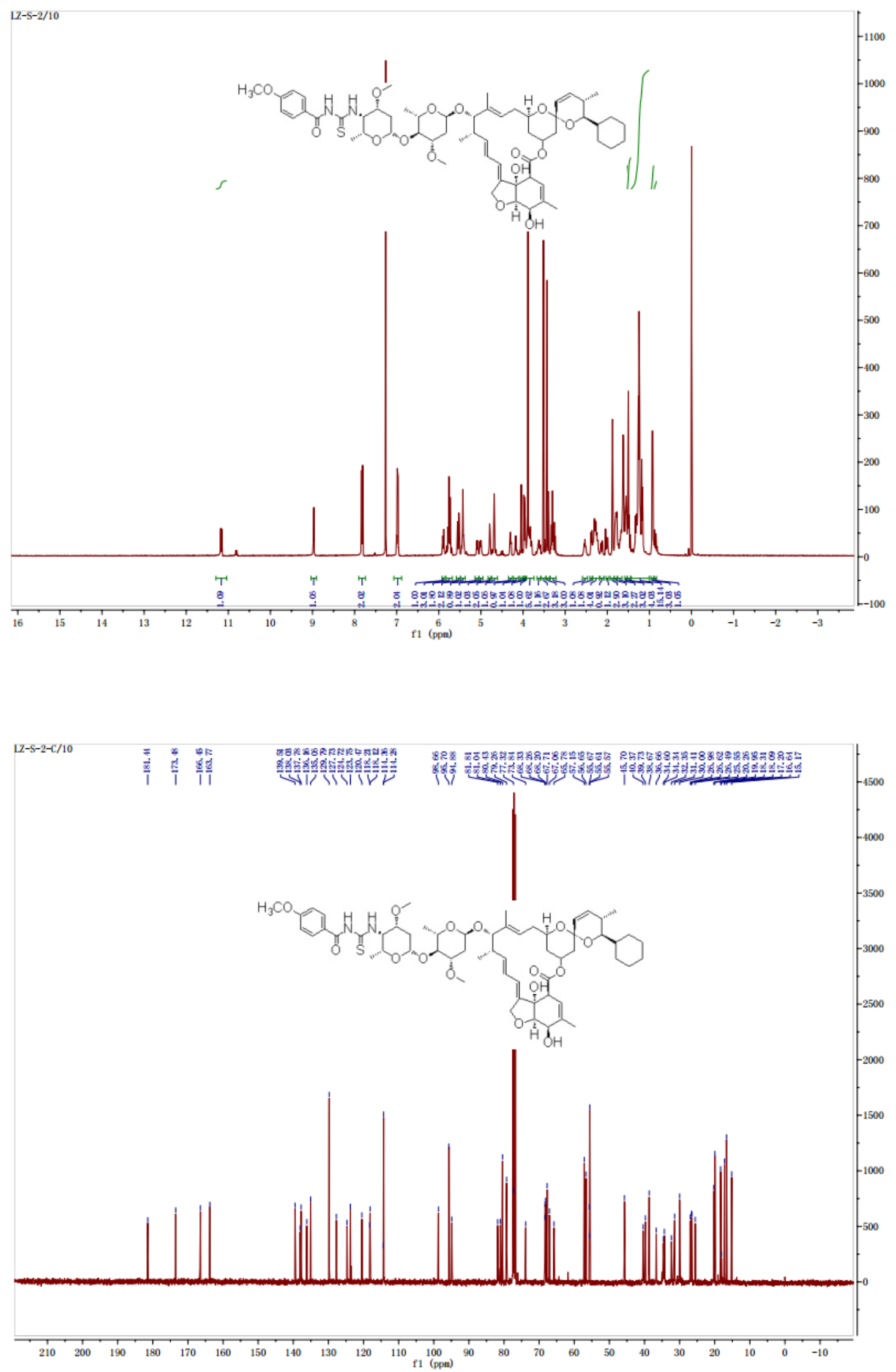
S-76: The ${ }^{1} \mathrm{H}-\mathrm{NMR}$ and ${ }^{13} \mathrm{C}-\mathrm{NMR}$ spectrums of $\mathbf{6 k}$
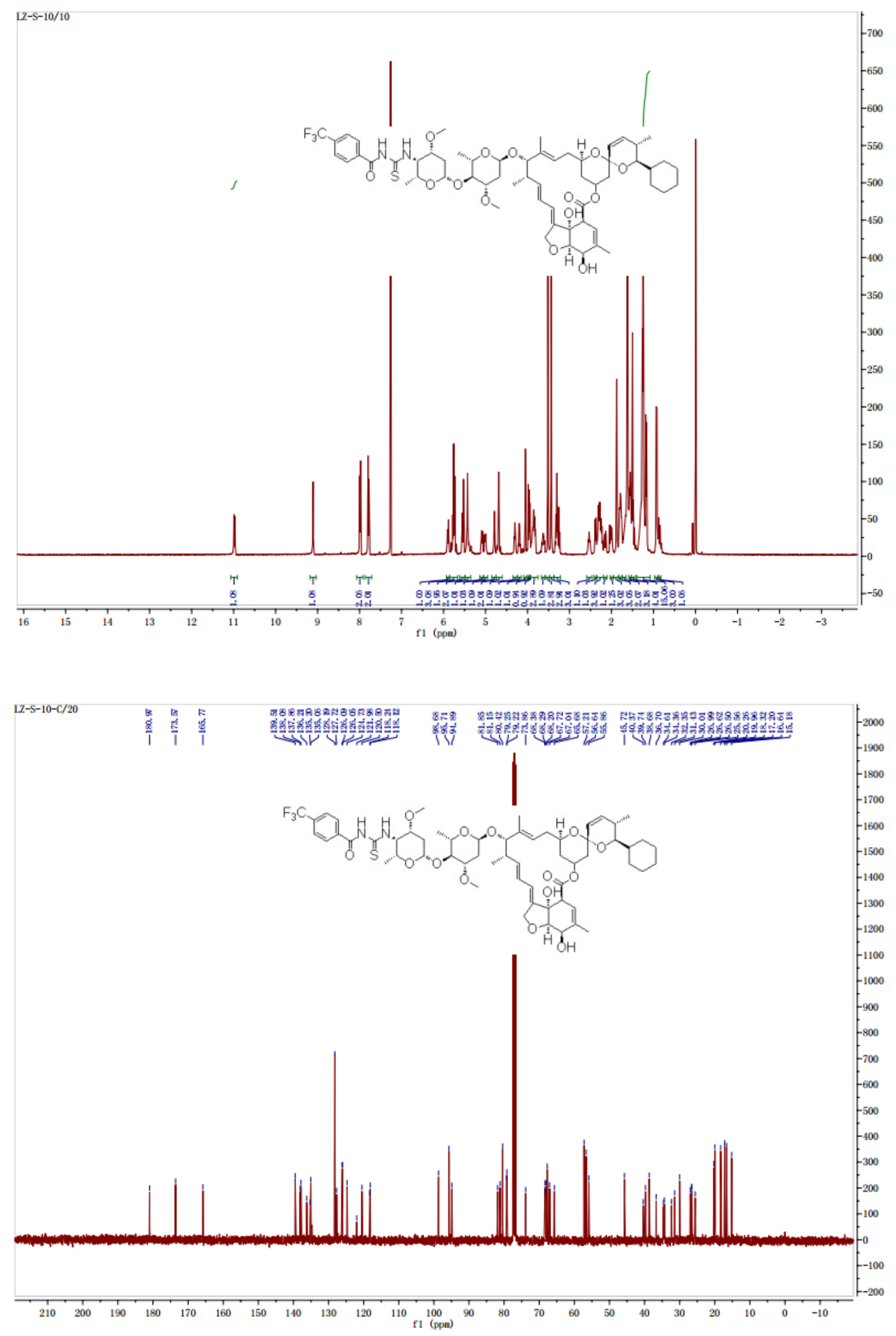
S-77: The ${ }^{1} \mathrm{H}-\mathrm{NMR}$ and ${ }^{13} \mathrm{C}-\mathrm{NMR}$ spectrums of $6 \mathbf{1}$
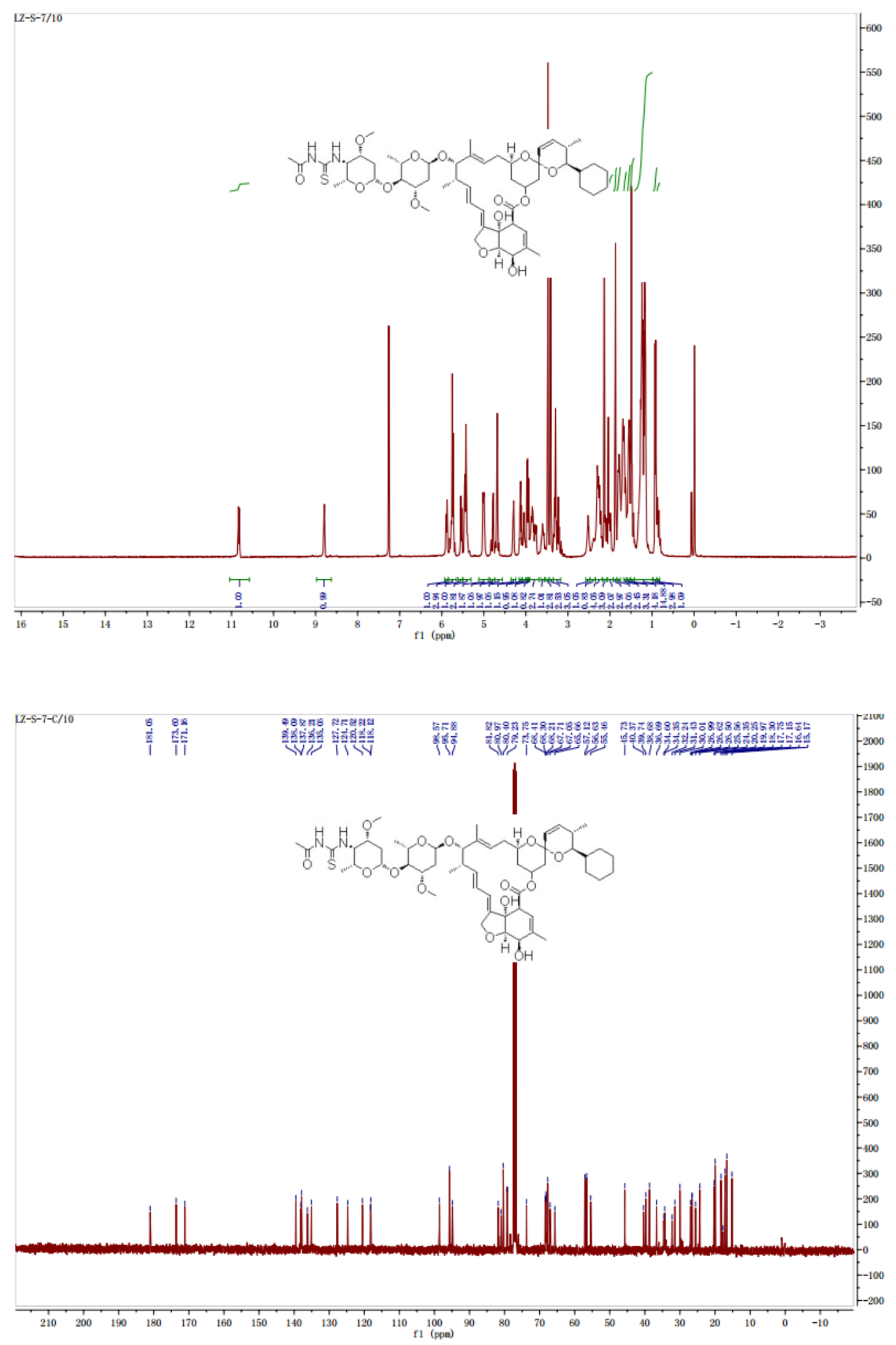
S-78: The ${ }^{1} \mathrm{H}-\mathrm{NMR}$ and ${ }^{13} \mathrm{C}-\mathrm{NMR}$ spectrums of $\mathbf{6 m}$
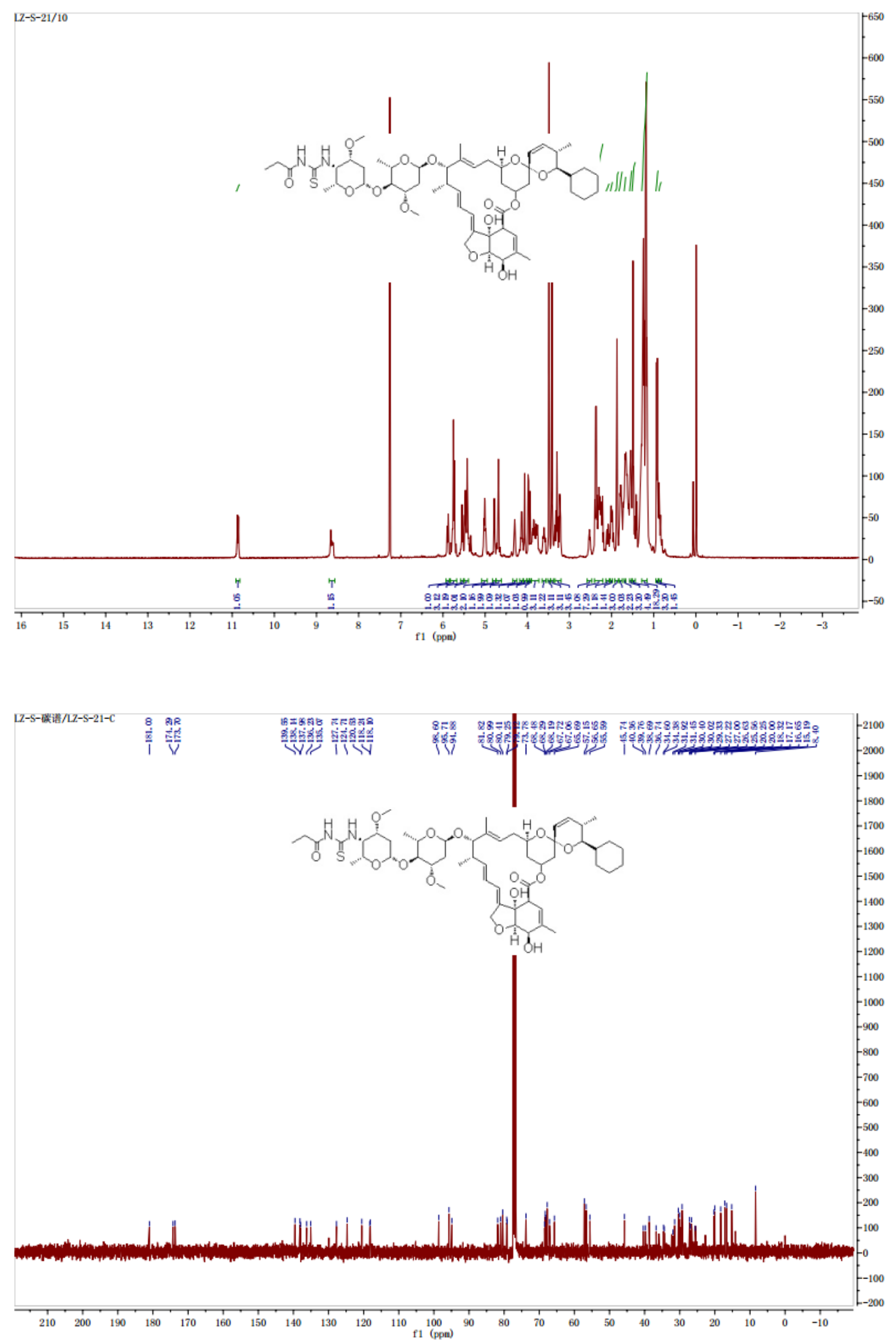
S-79: The ${ }^{1} \mathrm{H}-\mathrm{NMR}$ and ${ }^{13} \mathrm{C}-\mathrm{NMR}$ spectrums of $\mathbf{6 n}$
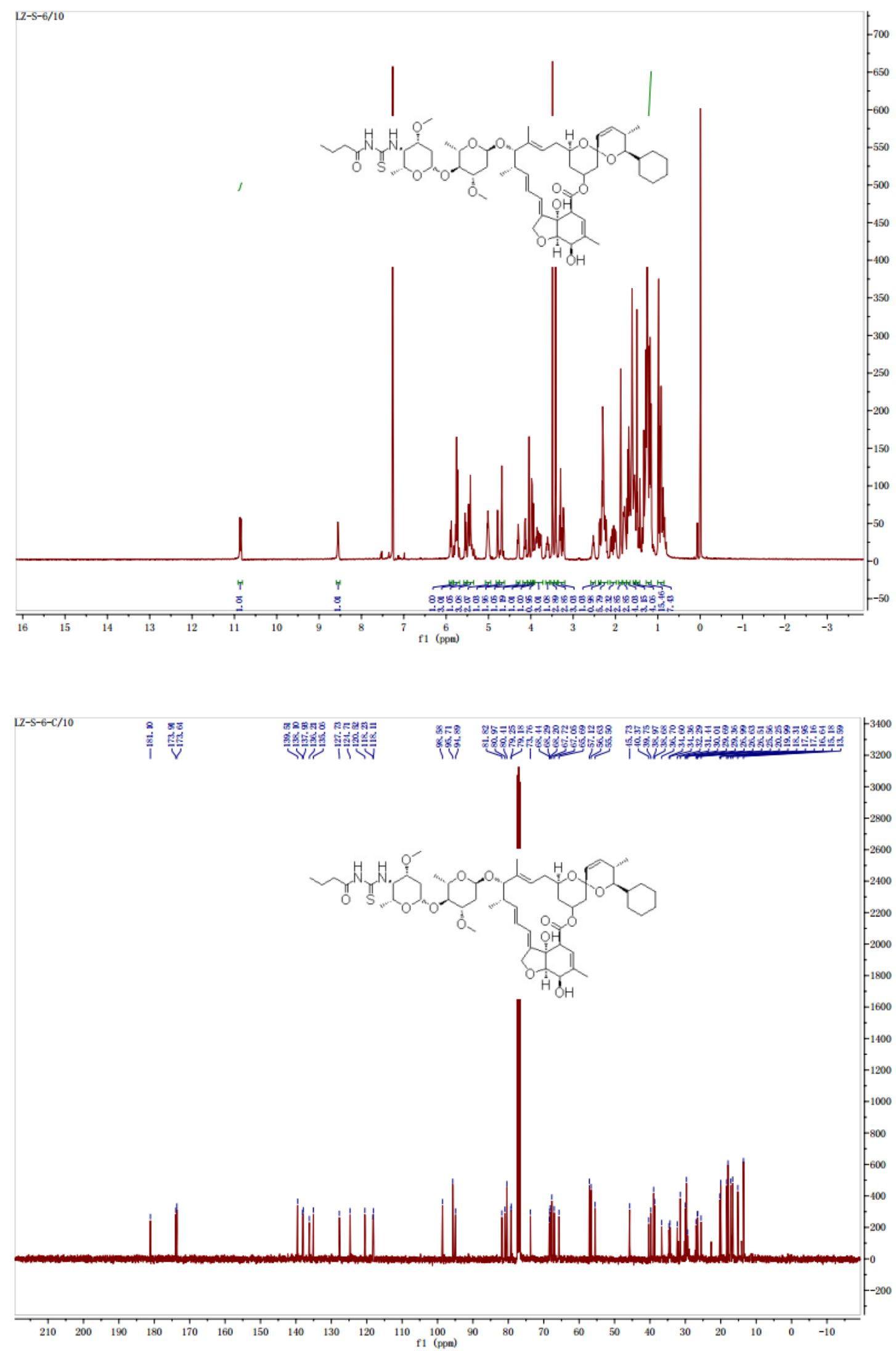
S-80: The ${ }^{1} \mathrm{H}-\mathrm{NMR}$ and ${ }^{13} \mathrm{C}-\mathrm{NMR}$ spectrums of $\mathbf{6 o}$
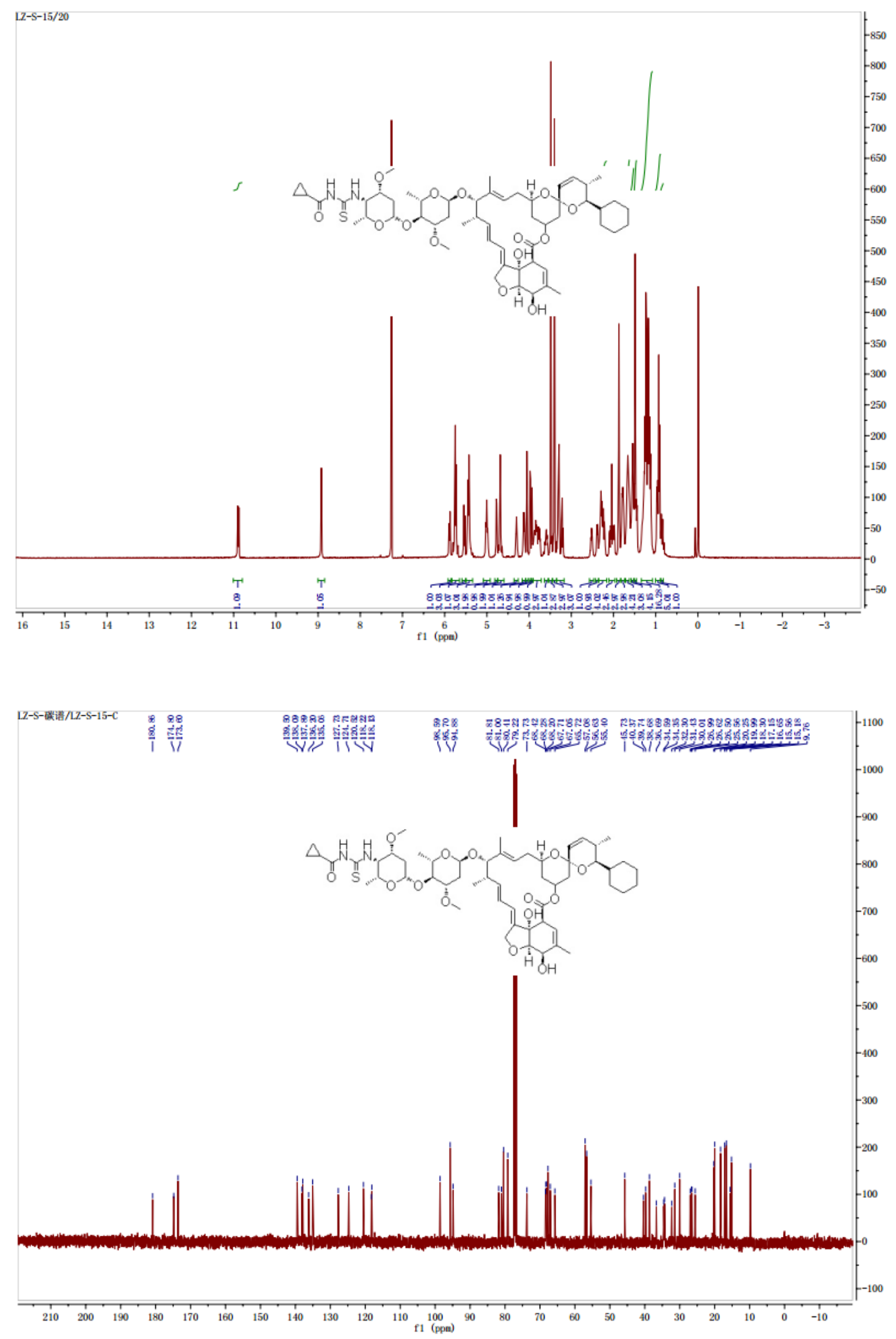
S-81: The ${ }^{1} \mathrm{H}-\mathrm{NMR}$ and ${ }^{13} \mathrm{C}-\mathrm{NMR}$ spectrums of $\mathbf{6 p}$
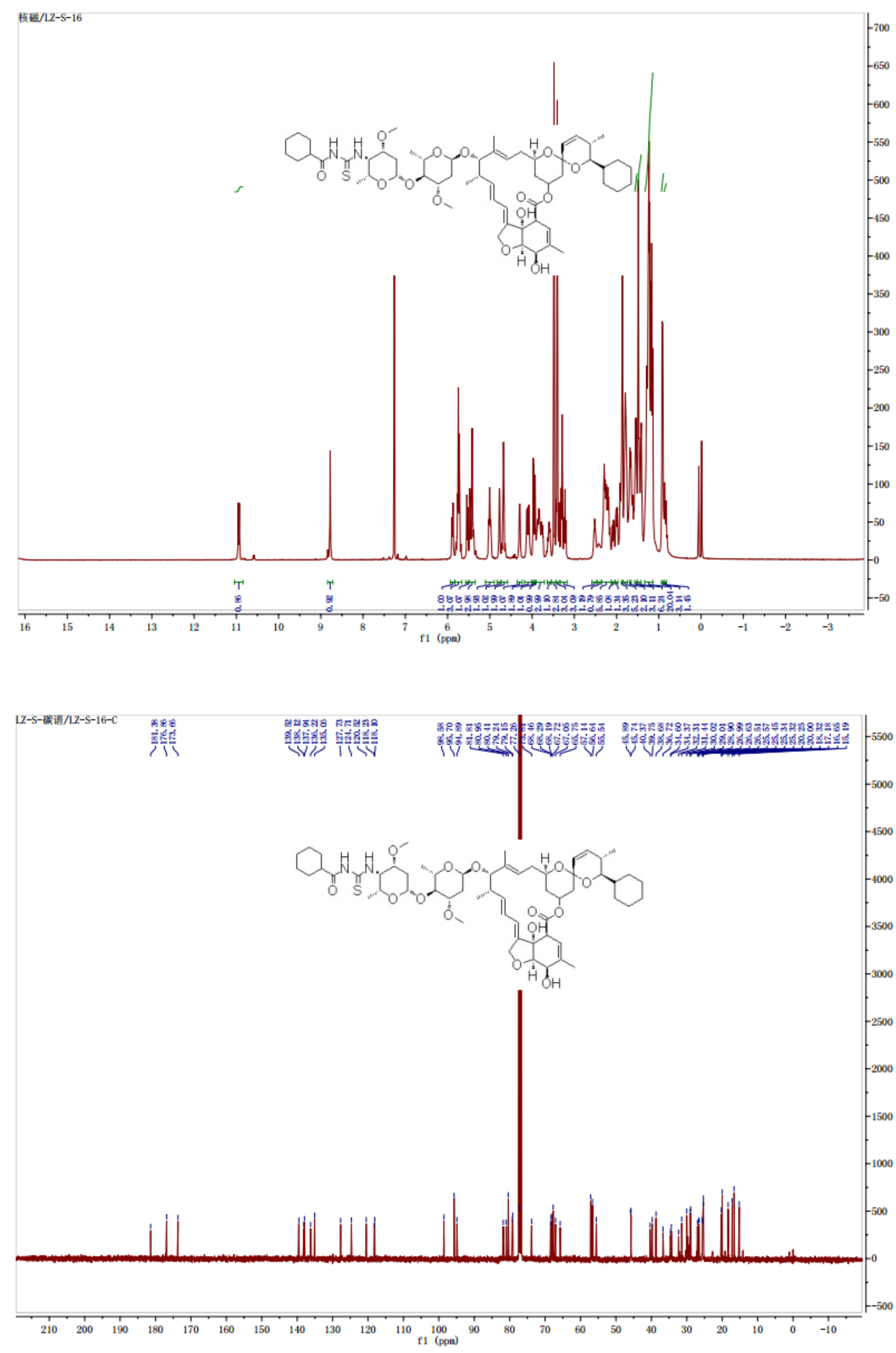
S-82: The ${ }^{1} \mathrm{H}-\mathrm{NMR}$ and ${ }^{13} \mathrm{C}-\mathrm{NMR}$ spectrums of $\mathbf{6 q}$
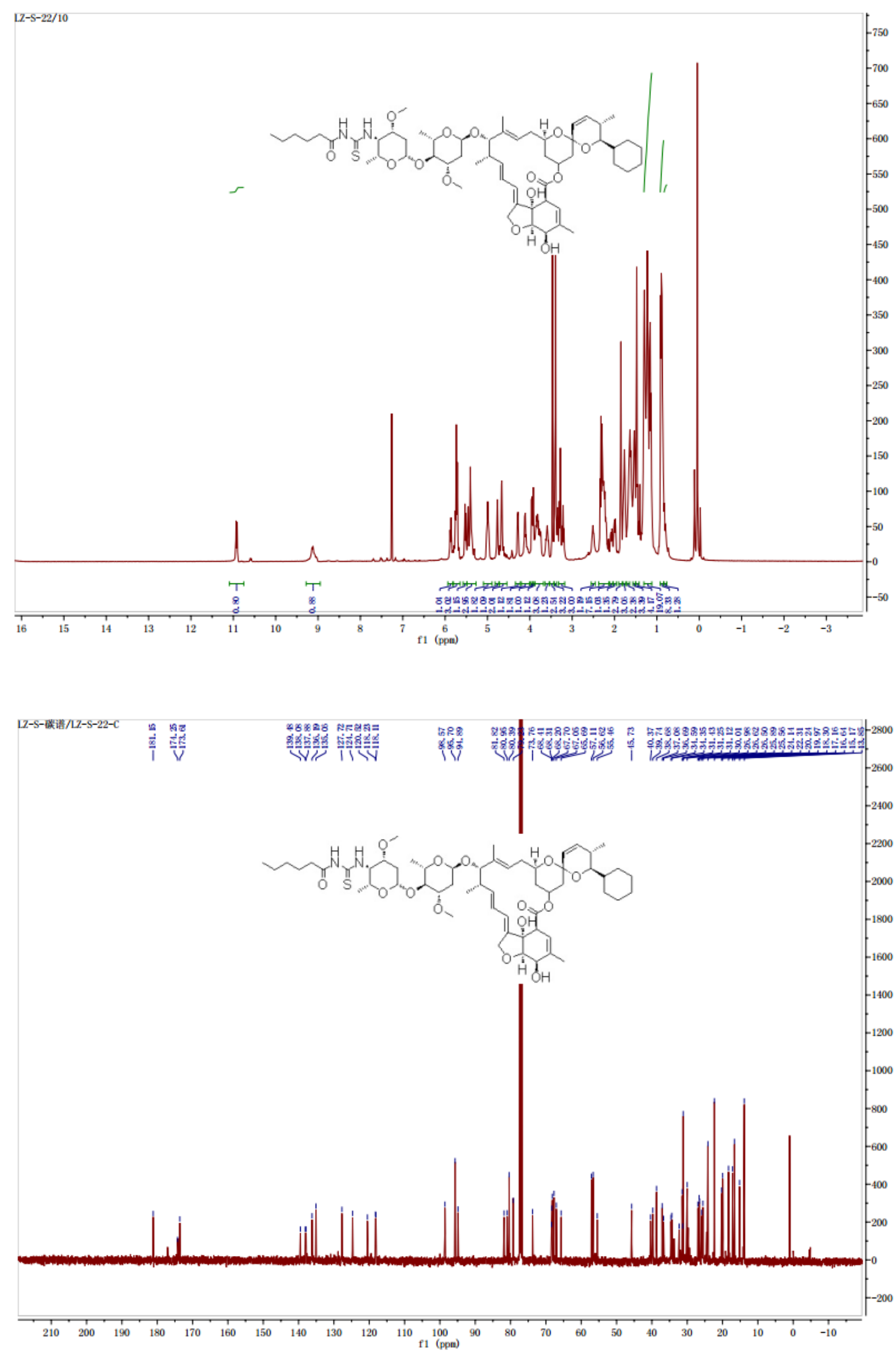
S-83: The ${ }^{1} \mathrm{H}-\mathrm{NMR}$ and ${ }^{13} \mathrm{C}-\mathrm{NMR}$ spectrums of $6 \mathbf{r}$
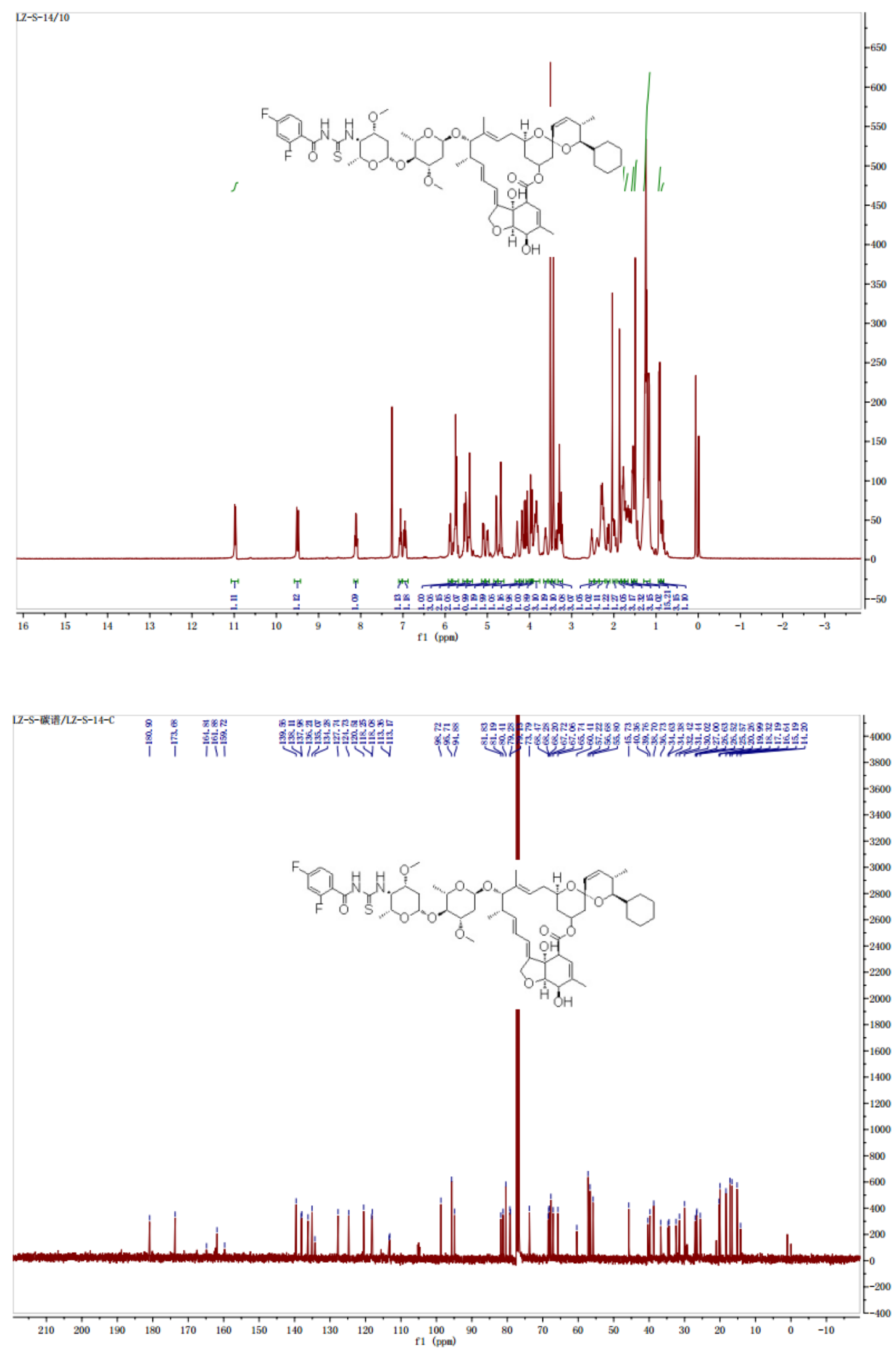
S-84: The ${ }^{1} \mathrm{H}-\mathrm{NMR}$ and ${ }^{13} \mathrm{C}-\mathrm{NMR}$ spectrums of $\mathbf{6 s}$
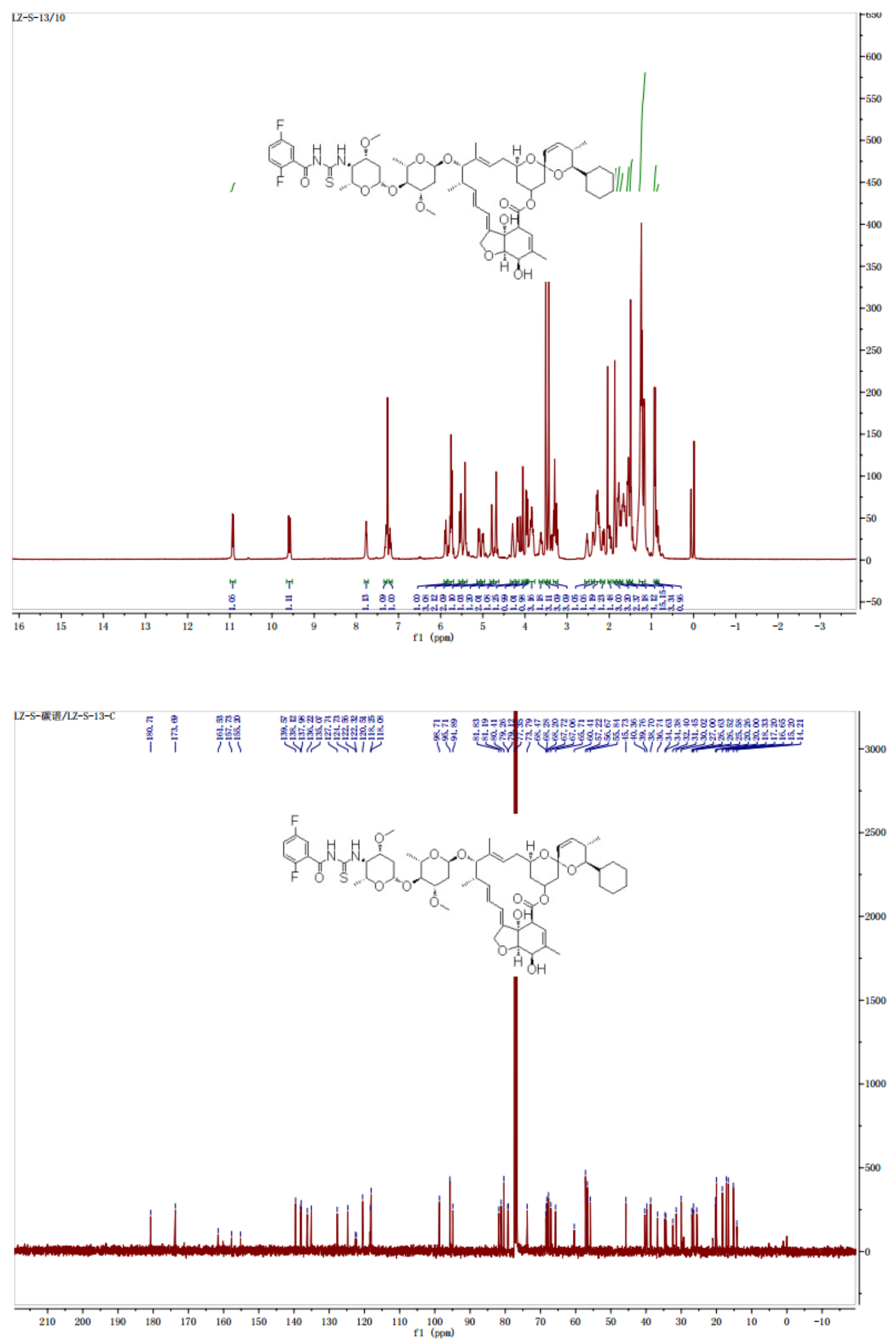
S-85: The ${ }^{1} \mathrm{H}-\mathrm{NMR}$ and ${ }^{13} \mathrm{C}-\mathrm{NMR}$ spectrums of $\mathbf{6 t}$
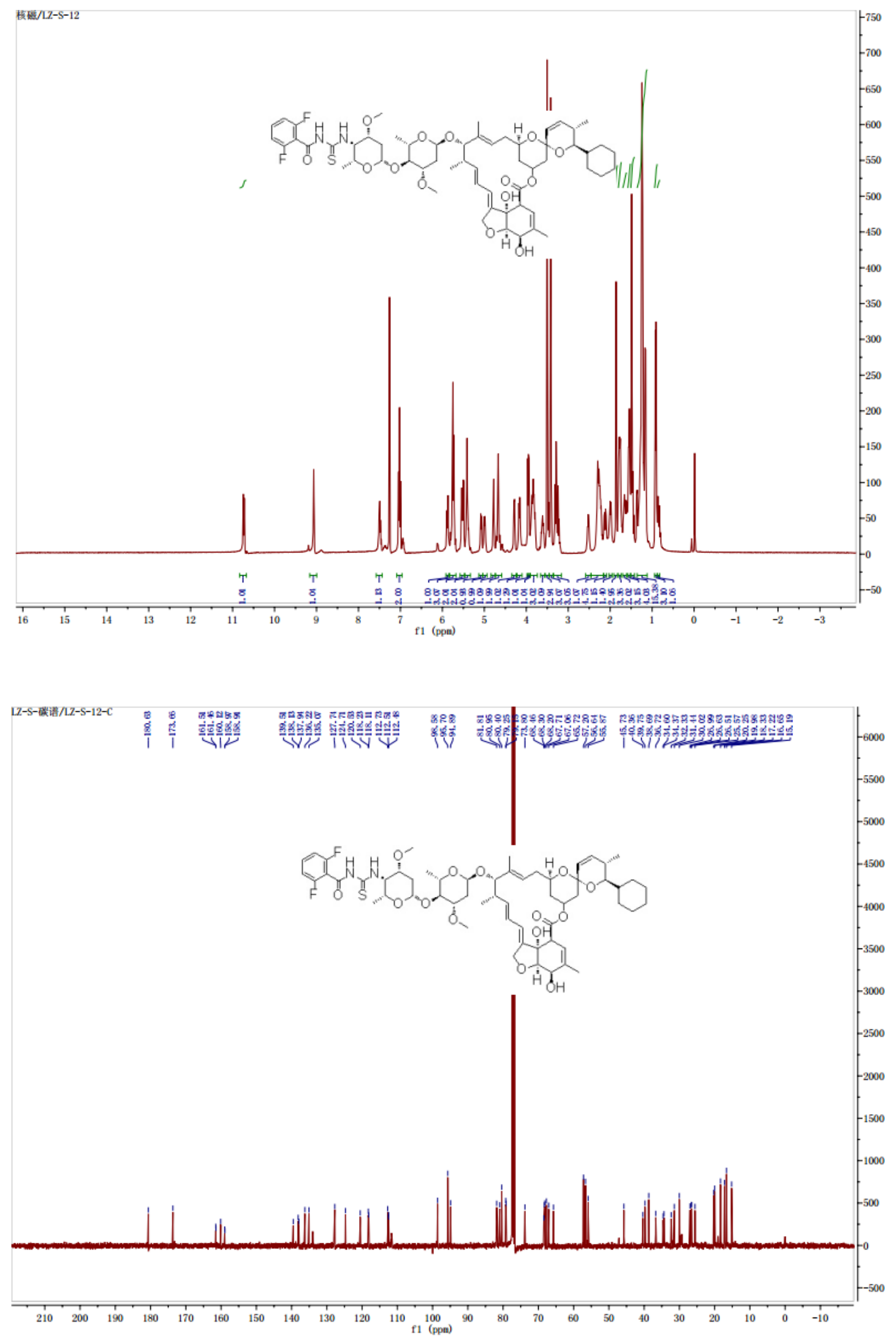
S-86: The ${ }^{1} \mathrm{H}-\mathrm{NMR}$ and ${ }^{13} \mathrm{C}-\mathrm{NMR}$ spectrums of $\mathbf{6 u}$
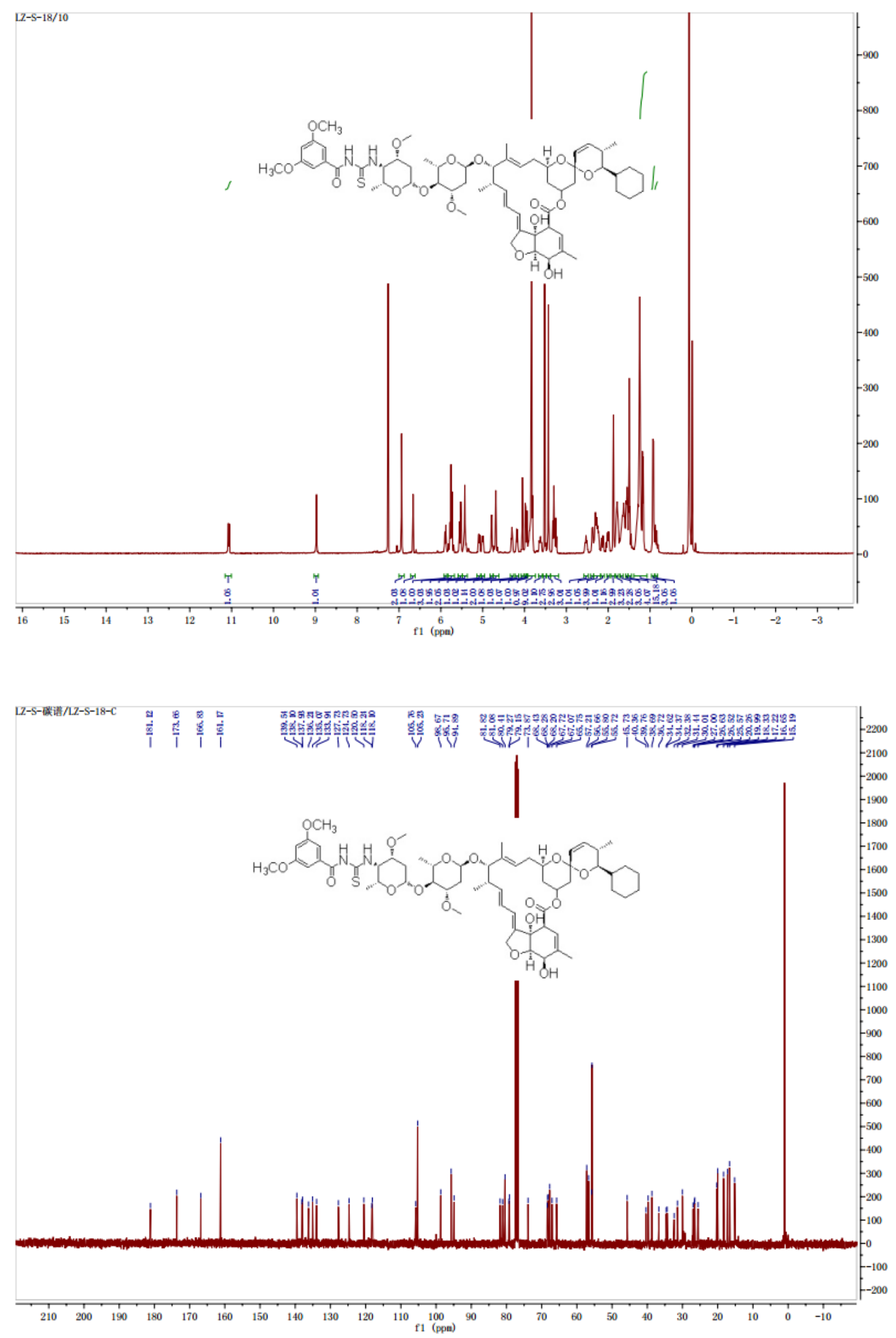
S-87: The ${ }^{1} \mathrm{H}-\mathrm{NMR}$ and ${ }^{13} \mathrm{C}-\mathrm{NMR}$ spectrums of $\mathbf{6 v}$
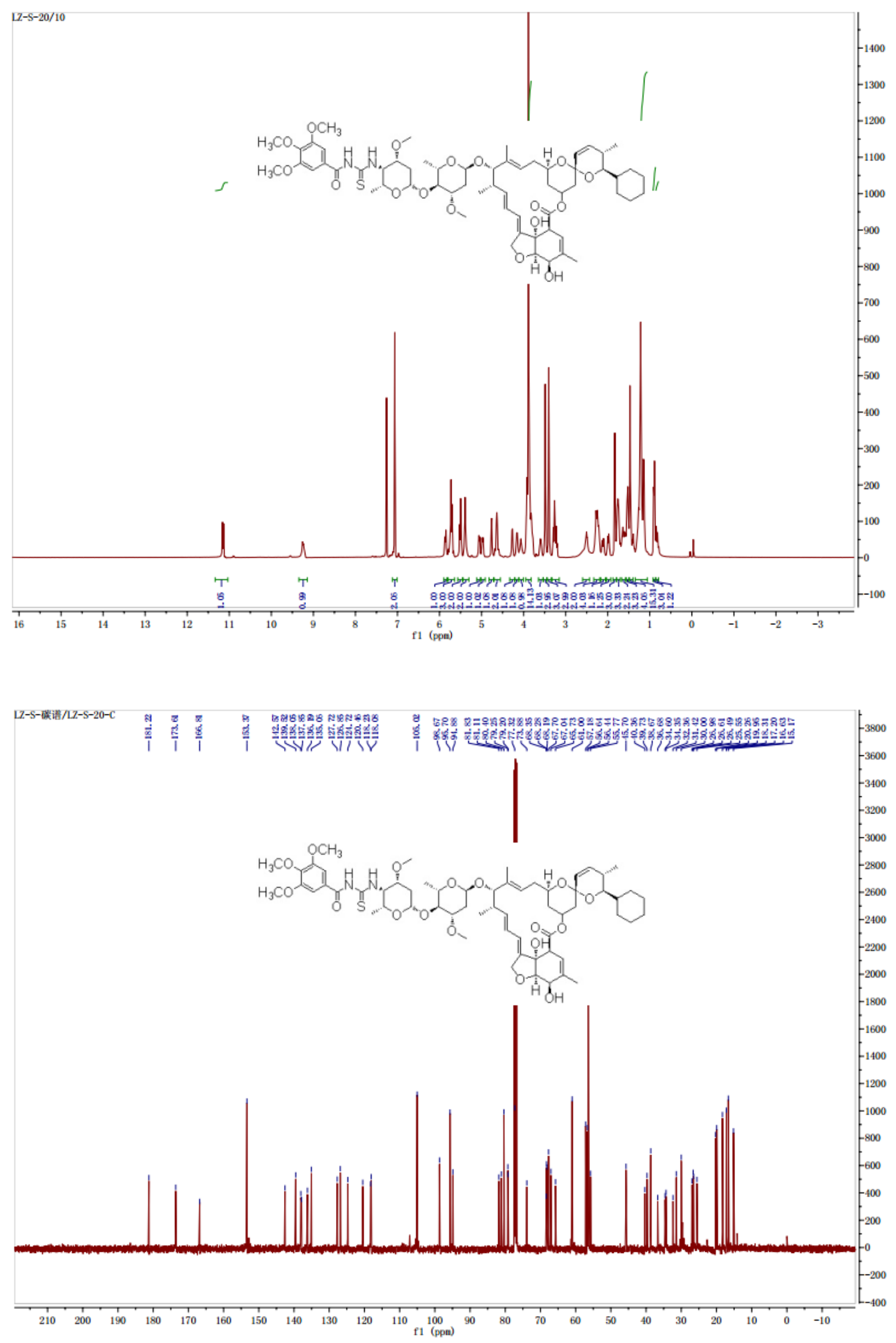